H. G. Bender

T. Schwenzer

H.-J. Weyergraf

D. Wallwiener

\section{Qualitätssicherung durch Kommentierung der amtlichen Gebührenordnung für Ärzte (GOÄ 96) im Bereich Operative Frauenheilkunde} Stellungnahme der GOÄ-Kommission der Deutschen Gesellschaft für Gynäkologie und Geburtshilfe zu den meist beanstandeten GOÄ-Ziffern bzw. Analogziffern

\section{Inkontinenz- und Deszensuseingriffe}

Die Inkontinenz- und Deszensuschirurgie war seit Inkrafttreten der GOÄ 1982 einem erheblichen Wandel ausgesetzt. Die GOÄ bildet praktisch nur die Leistungen Hysterektomie, vordere und hintere Scheidenplastik und Plastische Operation bei Harninkontinenz ab. Heute werden sehr differenziert unterschiedliche Teilschritte im operativen Gesamtkonzept eingesetzt und häufig miteinander kombiniert. Für viele Operationsverfahren gibt es in der GOÄ keine adäquaten Leistungsziffern, und diese neue Techniken stellen keine Modifikation bisheriger Verfahren dar.

Ziffer 1781 Operative Behandlung bei Harninkontinenz mittels Implantation eines künstlichen Schließmuskels

Die Ziffer ist für die Operation einer Harninkontinenz mittels Implantation eines künstlichen Schließmuskels berechnungsfähig, da es sich nicht um eine Teilleistung des hauptoperativen Eingriffes handelt, sondern um eine eigenständige operative Maßnahme.

Ziffer 3283 A Hohe Kuldoplastik nach McCall

Die modernen Erkenntnisse über die Ätiologie des Deszensus haben gezeigt, dass bei Zelenbildung der vorderen Vaginalwand häufig kein medialer, sondern ein lateraler Defekt der endopelvinen Faszie bzw. ihrer seitlichen Verankerung an der Beckenwand besteht. Gelegentlich bestehen mediale und laterale Defekte gleichzeitig. Für die lateralen Defekte wurden in den letzten Jahren vaginale und abdominale Operationsverfahren entwickelt, die nicht durch die vorhandenen Ziffern der GOÄ abgebildet werden. Daher ist eine Abrechnung über eine Analogziffer gemäß § 6 GOÄ zwingend geboten. Die Ziffer ist bei entsprechender Durchführung der Leistung neben anderen Operationsschritten, insbesondere auch neben den Ziffern 1125 bzw. 1127 GOÄ, berechenbar.

Die Bundesärztekammer hat in einer Stellungnahme aus April 1997 die Ziffer 3283 GOÄ analog für eine Douglasverödung nach Moszkowicz für berechenbar erachtet. Zur Begründung wurde ausgeführt, es handele sich hierbei um eine von der abdominalen Hysterektomie eigenständig indizierte und durchgeführte Leistung. Dementsprechend käme die Ziffer 3283 GOÄ analog zum Ansatz.

Ziffer 1126 Hintere Scheidenplastik mit Beckenbodenplastik

neben

Ziffer 1780 Plastische Operation zur Behebung der Harninkontinenz

Die Leistung nach Ziffer 1126 wird (überwiegend auf vaginalem Wege) bei einer Beckenbodeninsuffizienz, die Leistung nach Ziffer 1780 hingegen (überwiegend auf abdominalem Wege) aufgrund einer Harninkontinenz durchgeführt. Voraussetzung für die Nebeneinanderberechnungsfähigkeit beider Ziffern ist das Vorliegen von Harninkontinenz und Beckenbodeninsuffizienz. Besteht lediglich eine Harninkontinenz, kann selbstverständlich nur die Ziffer 1780 zum Ansatz kommen. 
Die Ziffer 1147 wird analog für die Scheidenfixation bzw. Vaginal Repair in Ansatz gebracht. Diese Leistung beurteilen wir als eine eigenständige Operation im Sinne der GOÄ. Insofern bestehen unsererseits keine Bedenken gegen den Ansatz der Nr.1147 analog - GOÄ.

Die Leistung ist, bei entsprechender Durchführung, neben der plastischen Operation bei Harninkontinenz gesondert berechnungsfähig.

\section{Mammachirurgie}

Die Aufstellung der GOÄ-Leistungsziffern stammt aus einer Zeit, seit der ein grundlegender Wandel in den Therapieverfahren stattgefunden hat. Daher treffen die Abrechnungspositionen in großen Teilen nicht mehr die heutigen standardmäßigen Operationsabläufe, die als zeitgemäß anzusehen sind. Dies gilt in besonders charakteristischer Weise für die heute üblichen Eingriffe an der Mamma. Während zum Zeitpunkt der Entwicklung der derzeit gültigen Gebührenordnung für Ärzte bei Mamma-Tumor-Operationen als Standard anzusehen war, dass zunächst eine Tumorexstirpation zur Malignitätssicherung vorgenommen wurde, die bei Tumornachweis zur modifiziert radikalen Mastektomie mit Ausräumung der ipsilateralen Axilla führte, wird nach den heutigen Maßstäben durchweg die so genannte brusterhaltende Mammakarzinom-Therapie bevorzugt durchgeführt. Bei diesem Vorgehen ist das notwendige Therapiekonzept, das sowohl Heilungssicherheit wie ästhetische Ansprüche erfüllt, wesentlich schwieriger als die standardmäßige ablative Therapie früherer Zeiten.

Ziffer 2580 A Freilegung und Schonung des N. intercostobrachialis

Im Gegensatz zur Entwicklungsphase der jetzt gültigen Gebührenordnung für Ärzte (GOÄ), wonach früher die Durchtrennung der Nerven Standard war, ist der Erhalt der Intercostobrachialnerven heute ein wichtiges Ziel des Eingriffes. Daher stellt die Schonung der einzelnen Nerven der Axilla im Rahmen einer Radikaloperation eine jeweils selbständige ärztliche Leistung dar und ist gem. § 6 der GOÄ zusätzlich berechnungsfähig.

Bei Durchtrennung dieser Nerven ist die Ziffer 2580 A nicht in Ansatz zu bringen. Die Ziffer ist nur in den Fällen berechnungsfähig, in denen eine Freilegung und Schonung des N. intercostobrachialis im Rahmen einer Radikaloperation, d.h. einer Ausräumung der Axillalymphknoten bis Level III, durchgeführt wird.

\section{Ziffer 2803 A Freilegung und Schonung der V. axillaris}

Diese Leistung ist nur berechnungsfähig, sofern eine Axillaausräumung bis Level III erfolgt. Im Rahmen dieses radikalen Vorgehens wird eine komplette Freipräparation und Schonung der Vena axillaris in besonderer Tiefe bis zum Hauptstamm durchgeführt. Bei entsprechender medizinische Indikation stellt die Leistung eigenständige operative Maßnahme dar und ist gem. §6 Abs. 2 der GOÄ zusätzlich berechnungsfähig.
Die Auffassung einiger Krankenversicherungen, wonach die Ziffer 2583 im Rahmen anderer Operationen grundsätzlich nicht gesondert berechnungsfähig wäre, trifft nicht zu. Es gibt durchaus Fälle, in denen die Leistung nach Ziffer 2583 als selbständige Leistung gewertet werden kann. Im Einzelfall sind Leistungsumfang und Schwierigkeitsgrad zu berücksichtigen.

Die Neurolyse des N. thoracicus dorsalis sowie des N. thoracicus longus ist in der Ziffer 2408 - Lymphstromausräumung der Axilla - mit beinhaltet. Eine Ausnahmesituation würde dann vorliegen, wenn eine ausgedehnte Metastasierung in der Axilla die Präparation dieser Nerven erheblich erschweren würde. Handelt es sich jedoch um einen makroskopisch negativen Lymphknotenbefund, muss man davon ausgehen, dass die Operation nicht erschwert war. Dementsprechend ist die Neurolyse dieser Nerven nicht gesondert abrechnungsfähig.

Die Neurolyse des N. intercosto brachialis kann unseres Erachtens sehr wohl diskutiert werden, da dieser Nerv früher oftmals durchtrennt wurde, heute aber, um die Sensibilität im Bereich des Arms zu erhalten, geschont wird. Die Präparation ist aufwendig und erschwert auch die Lymphonodektomie, selbst bei negativem Lymphknotenstatus. Eine Berechnung ist hier aus unserer Sicht möglich.

Abschließend kann daher festgehalten werden, dass unserer Ansicht nach die Neurolyse des $\mathrm{N}$. thoracicus dorsalis sowie des thoracicus longus nur bei Tumorummauerung abrechenbar ist. Die Neurolyse des N. intercostobrachialis halten wir hingegen auch bei negativem Lymphknotenstatus für berechnungsfähig.

\section{Endometrioseoperationen}

Leichtere Endometriosefälle profitieren von der endoskopischen Diagnostik, die so einer konsequenten Therapie zugeführt werden können. Die Endometriose nach dem ASF-Score I und II ist in den meisten Fällen laparoskopisch angehbar. Sehr leicht wird auch, vergleichbar zu laparoskopischen Adhäsiolyse, die Grenze des endoskopisch Sinnvollen überschritten und eine komplexe Endometriose, z. B. mit Befall der Ovarien oder des Septum rectovaginale, endoskopisch nur insuffizient therapiert und damit die Rezidivrate noch erhöht. Häufig ist die Methode bei fortgeschrittener Endometriose überfordert. Darüber hinaus führt die Gefahr der Verletzung der Nachbarorgane, insbesondere des Ureters, zu einer inakzeptablen Komplikationsdichte.

Im Rahmen des $\S 6$ GOÄ sind selbständige ärztliche Leistungen, die in das Gebührenverzeichnis noch nicht aufgenommen sind, entsprechend einer nach Art, Kosten- und Zeitaufwand gleichwertigen Leistung des Gebührenverzeichnisses zu berechnen. Um die Fertilität der Patientin erhalten zu können, ist eine möglichst radikale Entfernung der Endometriose erforderlich.

Ziffer 2404 A Endometrioseresektion im Bereich des inneren Genitale und des Peritoneums 
Ziffer 706 A Elektrochirurgische Laser-Endometrioseresektion bzw. Laserkoagulation zur Beseitigung von Stenosen oder zur Blutstillung bei endoskopischen Eingriffen

Die Leistung nach Nr. 706 steht für alle endoskopisch geführten Einsätze des chirurgischen Lasers. Insofern kann die Nr. 706 für Laser-gestützte Eingriffe aus allen Fachgebieten zum Ansatz kommen (z. B. Einsatz bei Ösophagustumor, Kehlkopfpolyp oder Prostata-Adenom). Trifft die in der Leistungslegende genannte Indikation („zur Beseitigung von Stenosen oder zur Blutstillung“) nicht zu und ist dennoch ein Lasereinsatz erforderlich, so kann die Nr. 706 analog berechnet werden (so auch die einschlägige Kommentierung zur GOÄ).

Insgesamt gehören Endometrioseoperationen, insbesondere wenn sie laparoskopisch durchgeführt werden, zu den anspruchvollsten gynäkologischen Maßnahmen. U.E. ist der zusätzliche Ansatz der Analogziffer 706 durchaus angemessen, wenn die Koagulation von kleinen Endometrioseherden, die diffus im Unterbauch einer Frau verteilt sein können, sehr aufwendig ist und eine entsprechende Dokumentation im Operationsbericht erfolgte.

\section{Operationen bei Myomen}

Die gesamte uteruserhaltende Myomchirurgie unterliegt einem strukturellen Wandel durch die Einführung laparoskopischer bzw. hysteroskopischer Operationstechniken. Durch die Perfektionierung der Präparationsinstrumente und die Möglichkeit der Morcellierung mittels elektronischer Morcellatoren ist die Größe des Myoms bei der laparoskopischen Myomenukleation kein Problem mehr. Vielmehr muss hier die Problematik gesehen werden, dass bei noch nicht erfülltem bzw. prospektivem Kinderwunsch die Rekonstruktion im Vordergrund steht. Gerade hier liegen die Grenzen der Methode. Bei zu tief intramural gelegenen Myomen ist oftmals eine suffiziente Rekonstruktion der Uterotomie nur sehr schwer möglich. Hier können im Extremfall Uterusrupturen bei nachfolgenden Schwangerschaften auftreten. Die hysteroskopische Resektion submuköser Myome stellt die minimalst invasive uteruserhaltende Myomoperation dar, wobei die ausgedehnte hysteroskopische Operation einen hohen Ausbildungsstand und eine akribische Berücksichtigung der Sicherheitsaspekte erfordert.

\section{Ziffer 1162 Abdominale Myomenukleation}

Je nach Schwierigkeitsgrad sind die nachfolgend beschriebenen Myomoperationen als eigenständige Eingriffe jeweils unabhängig voneinander und unabhängig von der Anzahl bei gleichen Myomsitz gesondert berechnungsfähig:

a) subseröse bzw. gestielte Myome ohne Notwendigkeit der Uterusrekonstruktion als solitäre Myome

b) solitäre oder multiple intramurale Myome mit der Notwendigkeit der zusätzlichen Uteruswandrekonstruktion nach Enukleation

c) intraligamentäre Myome

d) zervikale Myome, die nach intraperitoneal oder retroperitoneal entwickelt sind e) solitäre oder multiple Myome mit kompliziertem Sitz

f) sehr große intramurale Myome, die die komplette Wandung des Uterus durchsetzen und einen erheblichen Uteruswanddefekt darstellen

g) intrakavitäre submukös gestielte Myome

h) intrakavitäre submukös/intramurale Myome mit unterschiedlichen Myomanteil intramural

i) intravaginale Zervixkarzinome

\section{Ziffer 1800 A Morcellement}

Die Enukleation von Myomen auf endoskopischen Wege und deren anschließende Bergung aus der Bauchhöhle ist nur bei Durchführung einer Morcellierung möglich. Für die manuelle elektronische Morcellierung ist die Ziffer 1800 analog anzusetzen. Hierbei ist allerdings zu berücksichtigen, dass bei der endoskopischen Morcellierung eine Laparotomie nicht notwendig ist. Daraus folgt, dass in der Ziffer 1800 A die Leistung nach Ziffer 700 enthalten ist. Dementsprechend ist bei der endoskopischen manuellen elektronischen Morcellierung die Ziffer 700 in Abzug zu bringen.

\section{Hysterektomie}

Im Rahmen der Hysterektomie wegen gutartiger Erkrankungen kommt es immer wieder zu Problemen mit dem Zielleistungsprinzip. Die Ziffern 1138 und 1139 bilden die Hysterektomie mit oder ohne Adnexektomie, entweder vaginal oder abdominal, ab. Grundsätzlich sind nach dem Zielleistungsprinzip Nebenleistungen, die zur Erbringung der Hauptleistung zwingend erforderlich sind, in der Hauptleistung enthalten. Besondere Schwierigkeiten, die sich aus der Individualität des Einzelfalls ergeben, sind ggf. über die Steigerungssätze zu berücksichtigen. Im Rahmen dieser Überlegungen sind z.B. Verwachsungen des Uterus oder der Adnexe mit dem Darm in der Regel Teil der Zielleistung und somit nicht gesondert berechnungsfähig. In besonderen Fällen kann dennoch eine separate Berechnungsfähigkeit gegeben sein.

\section{Ziffer 1145 Operative Entfernung/Behandlung von Eierstock/ Eileiter einseitig}

Jede organerhaltende Maßnahme an einer Adnexe ist unserer Ansicht nach neben der Hysterektomie abrechnungsfähig, da in der Ziffer 1138 Eingriffe an den Adnexen nicht enthalten sind und die Hysterektomie in der Regel auch durchführbar wäre, ohne dass dieser Zusatzeingriff mit eigenständiger Indikationsstellung vorgenommen wird. Es handelt sich demnach um eine selbständige, gesondert berechnungsfähige Leistung.

\section{Diagnostische Laparoskopie}

Die GOÄ-Ziffer 700 beinhaltet die Durchführung einer diagnostischen Laparoskopie/Pelviskopie, ggf. mit PE. Hinsichtlich der Berechnungsfähigkeit dieser Leistung möchten wir vorab aus zwei entsprechenden Stellungnahmen der Bundesärztekammer zitieren: 
Stellungnahme der Bundesärztekammer vom 19.03.1992:

„.... Die auf Seite 11 des Protokolls zu TOP 2.2.15 aufgeführte Vorgehensweise, dass bei laparoskopischen Operationen gemäß den allgemeinen Bestimmungen vor Kapitel L die Nr. 3135 in Abzug gebracht werden sollte, ist nicht sachgerecht. Die Rechnungslegung sollte unter Anwendung der Nr. 700 GOÄ (Laparoskopie oder Nephroskopie, ggf. einschließlich PE, 800 Punkte) plus den entsprechenden Nummern des intraabdominalen Eingriffs ohne Abzug der Eröffnungsleistung erfolgen. Ausdrücklich wird klargestellt, dass neben der entsprechenden Position des intraabdominalen Eingriffes nur Nr. 700 GOÄ, nicht Nr. 701, berechnet werden kann...."

Stellungnahme der Bundesärztekammer vom 13.10.1992:

„... In der 2. Sitzung des Ausschusses „Gebührenordnung“ der Bundesärztekammer vom 30.01.1992 hat dieser zur GOÄ-Anwendung bei laparoskopischen Operationen Stellung genommen. Bei laparoskopischen Operationen ist danach grundsätzlich die Anwendung der Nr. 700 GOÄ plus den entsprechenden Nrn des intraabdominellen Eingriffs gegeben. Die Anwendung der Nr.315 neben der Nr. 700 bzw. 701 GOÄ ist u.E. nicht sachgerecht. Zwar sind bei laparoskopischen Operationen mehrere Zugänge erforderlich, diese sowie das Einbringen der Instrumente sind jedoch Bestandteil der Zielleistung und nicht eigenständig berechenbar. Werden mehrere Eingriffe in der Bauchhöhle in zeitlichem Zusammenhang laparoskopisch durchgeführt, so ist von der zusätzlich erbrachten Leistung die Eröffnungsleistung (Nr. 700 GOÄ) nicht abzuziehen. Dies ergibt sich daraus, dass hier nicht, wie bei der Schnittoperation, durch ein und denselben Schnitt wesentlich leichter mehrere Eingriffe in einer Sitzung durchgeführt werden können. Die Präambel zum Abschnitt L der GOÄ ist abgestimmt auf die Erbringung der Leistung als Schnittoperation. Die nur gering gegebenen Erleichterungen bei der Durchführung mehrerer laparoskopischer Operationen in einer Sitzung können sich allenfalls, falls dem nicht patientenbezogene Gründe entgegenstehen, auf den Steigerungsfaktor hinsichtlich der weiteren erbrachten Operationen auswirken..."

Die Besonderheiten einer - deutlich schwierigeren und zeitaufwendigeren - laparoskopischen Operation sind in der zur Zeit gültigen Gebührenordnung nicht berücksichtigt, so dass der Ausschuss „Gebührenordnung“ der Bundesärztekammer den Beschluss gefasst hat, dass die Rechnungslegung für derartige Eingriffe unter Anwendung der GOÄ Nr. 700 plus den entsprechenden Nummern des intraabdominalen Eingriffs unter Abzug der Eröffnungsleistung erfolgen sollte.

Dabei ist selbstverständlich auch zu berücksichtigen, dass vor Durchführung der eigentlichen Operation regelmäßig eine diagnostische Laparoskopie stattfindet. Aus unserer Sicht besteht somit kein Widerspruch zwischen der Auffassung der Bundesärztekammer und den von einigen privaten Krankenversicherungen angesprochenen Passagen in der GOÄ-Kommentierung von Brück.

Ziffer 1155 Pelviskopie mit Anlage eines druckkontrollierten Pneumoperitoneums und Anlegen eines Portioadapters neben Ziffer 700 - Laparoskopie
Die Ziffern 1155 und 700 halten wir beispielsweise nebeneinander berechnungsfähig, wenn zunächst eine diagnostische Laparoskopie mit Anlage eines druckkontrollierten Pneumoperitoneums durchgeführt wurde und sodann der vaginale Eingriff (z.B. Hysterektomie, hohe Kuldoplastik nach McCall) mit anschließender Kontroll-Pelviskopie nebst erneuter Anlage eines druckkontrollierten Pneumoperitoneums erfolgt.

\section{Ziffer 5030 A Videodokumentation}

In Anlehnung an die GOÄ-Kommentierung von Brück halten wir das Video-Monitoring für nicht gesondert berechnungsfähig. Eine darüber hinausgehende Dokumentation kann jedoch analog nach Nr. 5030 zum Einfachsatz berechnet werden.

\section{7. weitere operative Leistungen}

Ziffer 2802 Freilegung und Unterbindung eines Blutgefäßes in der Brust- oder Bauchhöhle

Die Auffassung einiger Krankenversicherungen, wonach die Ziffer 2802 im Rahmen anderer Operationen grundsätzlich nicht berechnungsfähig ist, trifft nicht zu. Es gibt durchaus Fälle, in denen die Leistung nach Ziffer 2802 als selbständige operative Maßnahme durchgeführt wird, beispielsweise, wenn es sich aufgrund bestehender Tumorinfiltration um eine besonders schwierige und örtlich besonders aufwändige Gefäßfreilegung handelte. Im Einzelfall sind Leistungsumfang und Schwierigkeitsgrad zu berücksichtigen.

Ferner kann die Ziffer zusätzlich zum Ansatz kommen, wenn im Rahmen einer Lymphadenektomie (Ziffer 1809 GOÄ) schwierige und aufwändige Gefäßfreilegungen erforderlich werden. In solchen Fällen handelt es sich u.E. nicht um eine Teilleistung des hauptoperativen Eingriffes, sondern um eine gesondert berechenbare Leistung.

\section{Ziffer 3172 Operative Darmmobilisation}

Wir halten den Ansatz der Ziffer 3172 für gerechtfertigt, sofern es sich um eine langwierige, schwierige operative Leistung handelte.

Die Ziffer kommt unserer Ansicht nach ebenfalls im Rahmen eines Rezidiveingriffs zum Ansatz. Hierzu verweisen wir auch auf die Beschlüsse des Konsultationsausschusses der Bundesärztekammer im Bereich Herzchirurgie, wonach die Pericardiolyse bei Zustand nach Voroperation, Zustand nach Bestrahlung oder Zustand nach Entzündung gesondert berechnungsfähig ist. Gleiches muss für den Bereich der operativen Gynäkologie gelten.

Ziffer 1829 Ureterolyse bei retroperitonealer Fibrose und ggf. intraperitonealen Verwachsungen des Harnleiters

Gemäß der GOÄ-Leistungslegende ist die Harnleiterfreilegung bei Vorliegen einer retroperitonealen Fibrose und bei intraperitonealen Verwachsungen des Harnleiters mit der Ziffer 1829 in Ansatz zu bringen. 
Die Nr. 1829 trägt nicht den Zusatz „als selbständige Leistung“, so dass deren Abrechnung nicht nur bei der Behandlung des Ormond-Syndroms, sondern auch bei Beseitigung ausgedehnter retroperitonealer Verwachsungen im Rahmen eines anderen operativen Eingriffs infrage kommt.

Unserer Ansicht nach ist der Ansatz der Ziffer 1829 bei Eingriffen gerechtfertigt, bei denen aus dem Operationsbericht zu entnehmen ist, dass eine eigene Indikation zur Durchführung der Ureterolyse vorliegt - beispielsweise, falls bei entzündlichen Veränderungen, Tumorinfiltration oder ausgedehnten Verwachsungen es einen hohen Zeitaufwand, Risiko und Mühe erfordert, den Ureter langstreckig freizulegen. In diesen Fällen ist die Ziffer 1829 für eine eigenständige operative Maßnahme gesondert in Ansatz zu bringen.

\section{Operative Therapie gutartiger Erkrankungen}

\section{Hysterektomie \\ Vorbemerkung}

Im Rahmen der Hysterektomie wegen gutartiger Erkrankungen kommt es immer wieder zu Problemen mit dem Zielleistungsprinzip. Die Ziffern 1138 bzw. 1139 GOÄ bilden die Hysterektomie mit oder ohne Adnexe entweder vaginal oder abdominal ab. Grundsätzlich sind nach dem Zielleistungsprinzip Nebenleistungen, die zur Erbringung der Hauptleistung zwingend erforderlich sind, in der Hauptleistung enthalten. Besondere Schwierigkeiten, die sich aus der Individualität des Einzelfalls ergeben, sind ggf. über die Steigerungssätze zu berücksichtigen. Im Rahmen dieser Überlegungen sind z. B. Verwachsungen des Uterus oder der Adnexe mit dem Darm i.d.R. Teil der Zielleistung und somit nicht gesondert berechnungsfähig. In besonderen Fällen kann dennoch eine separate Berechnungsfähigkeit gegeben sein (Tab.1).

\section{Schwierigkeiten bei der Erstattung}

\section{Ziffer 1145 bzw. 1146 neben Ziffer 1138 GOÄ}

Ziffer 3172 bzw. 3166 neben Ziffer 1138 bzw. 1139 GOÄ

Die Selbständigkeit der Ziffern 1145 bzw. 1146 neben der Ziffer 1138 GOÄ und der Ziffern 3172 bzw. 3166 neben der Ziffern 1138 bzw. 1139 GOÄ wird bemängelt. Die Krankenversicherungen behaupten, dass die Leistungen nach Ziffer 1145 bzw. 1146 in der Leistung nach Ziffer 1138 GOÄ und die Leistungen nach Ziffer 3172 bzw. 3166 in der Leistung nach Ziffer 1138 bzw. 1138 GOÄ enthalten sind.

\section{Kommentar zur Ziffer 1145 bzw. 1146 neben Ziffer 1138 *1 GOÄ} Jede organerhaltende Maßnahme an einer Adnexe ist neben der Hysterektomie abrechnungsfähig, da in der Ziffer 1138 GOÄ Eingriffe an den Adnexen nicht enthalten sind und die Hysterektomie in der Regel auch durchführbar wäre, ohne dass dieser Zusatzeingriff mit eigenständiger Indikationsstellung vorgenommen wird.

\section{Kommentar zur Ziffer 1829a neben Ziffer 1138 bzw. 1139 GOÄ}

In bestimmten Fällen ist neben der Hysterektomie als selbständige Leistung eine Ureterolyse erforderlich. Dies kann z.B. bei Endometriose, intraligamentären Myomen, Verwachsungen etc. zur Behebung oder Vorbeugung einer Harnstauungssymptoma-
Tab. 1

\begin{tabular}{|c|c|c|c|}
\hline Ziffer & Leistungstext & Punkte & EURO \\
\hline $\begin{array}{l}1138 \\
* 1\end{array}$ & $\begin{array}{l}\text { Vaginale/abdominale Totalexstirpation } \\
\text { des Uterus ohne Adnexentfernung }\end{array}$ & 2770 & 161,46 \\
\hline 1139 & $\begin{array}{l}\text { Vaginale/abdominale Totalexstirpation } \\
\text { des Uterus mit Adnexentfernung }\end{array}$ & 3330 & 194,10 \\
\hline \multirow[t]{2}{*}{$\begin{array}{l}1145 \\
* 1\end{array}$} & $\begin{array}{l}\text { Ovarektomie, Ovariotomie, Salpingekto- } \\
\text { mie, Salpingotomie, Salpingolyse und/ } \\
\text { oder Neostomie durch vaginale oder } \\
\text { abdominale Eröffnung der Bauchhöhle, } \\
\text { einseitig }\end{array}$ & 1660 & 96,76 \\
\hline & oder & & \\
\hline $\begin{array}{l}1146 \\
* 1\end{array}$ & $\begin{array}{l}\text { Ovarektomie, Ovariotomie, Salpingekto- } \\
\text { mie, Salpingotomie, Salpingolyse und/ } \\
\text { oder Neostomie durch vaginale oder } \\
\text { abdominale Eröffnung der Bauchhöhle, } \\
\text { beidseitig }\end{array}$ & 2220 & 129,40 \\
\hline $1829 a$ & Ureterolyse, als selbständige Leistung & 1110 & 64,70 \\
\hline \multirow[t]{2}{*}{3172} & $\begin{array}{l}\text { Operative Darmmobilisation bei Ver- } \\
\text { wachsungen, als selbständige Leistung }\end{array}$ & 1600 & 93,26 \\
\hline & oder & & \\
\hline 3166 & $\begin{array}{l}\text { Operative Beseitigung von Atresien, } \\
\text { Stenosen (Septen) und/oder Divertikeln } \\
\text { des Jejunums oder des lleums }\end{array}$ & 3000 & 174,86 \\
\hline 2015 & $\begin{array}{l}\text { Anlegen einer oder mehrerer Redon- } \\
\text { drainagen (vaginal) }\end{array}$ & 0060 & 3,50 \\
\hline 2015 & $\begin{array}{l}\text { Anlegen einer oder mehrerer Redon- } \\
\text { drainagen (abdominal) }\end{array}$ & 0060 & 3,50 \\
\hline
\end{tabular}

tik notwendig sein. Die Ureterolyse, die ein- oder beidseitig notwendig werden kann, erfährt dann eine selbständige Indikationsstellung. Sie ist nicht notwendiger Bestandteil der Hysterektomie. Diese Leistung könnte auch ohne Freilegung der Harnleiter erfolgen.

\section{Kommentar zur Ziffer 3172 bzw. 3166 neben Ziffer 1138 bzw. 1139 GOÄ}

Bei chronischem Verwachsungsbauch (z.B. nach multiplen Voroperationen) erfolgt die Laparotomie häufig wegen der chronischen Beschwerden wie Schmerzen, Subileus etc. In diesen Fällen ist oft das gesamte Darmkonvolut zu einem Konglomerat verwachsen bzw. stenosiert, und es muss eine systematische, häufig über mehrere Stunden dauernde, Adhäsiolyse erfolgen. Diese Leistung stellt einen eigenständigen Teilschritt der Operation dar. Wenn im Rahmen einer solchen derart ausgedehnten Adhäsiolyse bzw. Aufhebung von Stenosierungen mit einer eigenständigen Indikationsstellung auch noch die Hysterektomie mit oder ohne Adnexe erfolgt, sind beide Ziffern nebeneinander abrechnungsfähig. Die Ziffer kann nicht in Ansatz gebracht werden, wenn es sich lediglich um einzelne Verwachsungsstränge handelt, in die z. B. Uterus und Adnexe einbezogen sind. Die Adhäsiolyse ist hier Bestandteil der Zielleistung und kann ggf. über den Steigerungsfaktor berücksichtigt werden. 


\section{Inkontinenz- und Deszensuseingriffe Vorbemerkung}

Die Inkontinenz- und Deszensuschirurgie war seit Inkrafttreten der GOÄ 1982 einem erheblichen Wandel ausgesetzt. Die GOÄ bildet praktisch nur die Leistungen Hysterektomie, vordere und hintere Scheidenplastik und Plastische Operation bei Harninkontinenz ab. Heute werden sehr differenziert unterschiedliche Teilschritte im operativen Gesamtkonzept eingesetzt und häufig miteinander kombiniert. Für viele Operationsverfahren gibt es in der GOÄ keine adäquaten Leistungsziffern, und diese neuen Techniken stellen keine Modifikation bisheriger Verfahren dar (Tab. 2).

Tab. 2

\begin{tabular}{|c|c|c|c|}
\hline Ziffer & Leistungstext & Punkte & EURO \\
\hline \multirow[t]{2}{*}{1125} & Vordere Scheidenplastik & 0924 & 53,86 \\
\hline & oder & & \\
\hline \multirow[t]{2}{*}{1126} & $\begin{array}{l}\text { Hintere Scheidenplastik mit Becken- } \\
\text { bodenplastik }\end{array}$ & 1290 & 75,19 \\
\hline & oder & & \\
\hline 1127 & $\begin{array}{l}\text { Vordere und hintere Scheidenplastik } \\
\text { mit Beckenbodenplastik }\end{array}$ & 1660 & 96,76 \\
\hline 1780 & $\begin{array}{l}\text { Plastische Operation zur Behebung der } \\
\text { Harninkontinenz }\end{array}$ & 1850 & 107,83 \\
\hline 1147 & $\begin{array}{l}\text { vaginale sacrospinale Fixation der Schei- } \\
\text { de nach Amreich Richter, abdominale } \\
\text { Promontorofixation der Scheide etc. }\end{array}$ & 1480 & 86,27 \\
\hline 3231 & $\begin{array}{l}\text { analog für Operation einer Enterocele, } \\
\text { Douglasverödung entspricht Operation } \\
\text { des Mastdarmvorfalles bei Zugang vom } \\
\text { After aus oder perianal, §6 }\end{array}$ & 1150 & 67,03 \\
\hline 3283 & $\begin{array}{l}\text { analog für paravaginale Scheidenplastik } \\
\text { (Paravaginal repair), Kuldoplastik nach } \\
\text { McCall entspricht Operation eines Nabel- } \\
\text { oder Mittellinien- oder Bauchnarben- } \\
\text { bruches, § } 6\end{array}$ & 1110 & 64,70 \\
\hline
\end{tabular}

\section{Schwierigkeiten bei der Erstattung}

Ziffer 1780 neben Ziffer 1127

Ziffer 3231 analog, Ziffer 3283 analog Ziffer 1147

Die Selbständigkeit der Ziffer 1780 neben 1127 wird von den Krankenversicherungen bestritten.

Die Krankenversicherungen behaupten, dass die Ziffern 3231 analog und 3283 analog keine selbständigen ärztlichen Leistungen gemäß § 6 GOÄ (Analogie) darstellen.

Die medizinische Notwendigkeit der Leistung nach Ziffer 1147 GOÄ in Verbindung mit der Leistung nach Ziffer 1127 wird von den Krankenversicherungen nicht anerkannt.

\section{Kommentar zur Ziffer 1780 GOÄ}

Diese Ziffer bildet die Leistung typischer Inkontinenzoperationen wie Kolposuspension nach Burch, Hirsch, Marshall-Marchetti etc., aber auch von Nadelsuspension, Schlingenoperation usw. ab. Die vordere Scheidenplastik wird als Inkontinenzoperation zunehmend verlassen und gilt heute nur noch als Deszensusope- ration. Wenn die vordere Scheidenplastik als Inkontinenzoperation durchgeführt wird, kann die Ziffer 1780 GOÄ angesetzt werden.

In den Fällen, in denen zusätzlich zu einer vorderen Scheidenplastik eine spezielle Inkontinenzoperation vorgenommen wird, kann selbstverständlich neben der Ziffer 1125 GOÄ (bzw. 1127 GOÄ) die Ziffer 1780 abgerechnet werden. Dies ergibt sich schon alleine daraus, dass für die Inkontinenzoperation ein anderer $\mathrm{Zu}$ gang erfolgen muss (z.B. bei der typischen Kombination einer vorderen Scheidenplastik (vaginal) mit der Kolposuspension nach Burch (abdominal). Die medizinische Notwendigkeit dieser Leistungen ist in der Diagnose zu beschreiben, Stressinkontinenz und Scheidenprolaps.

\section{Kommentar zur Ziffer 1147 GOÄ}

Die Scheidenfixation am Liq. sacrospinale nach Amreich Richter, die beim Prolaps des Scheidenblindsackes, aber auch beim Prolaps der Gebärmutter im Anschluss an die Hysterektomie erfolgen kann, stellt ein eigenständiges Operationsverfahren dar, das zusätzlich zu den üblichen plastischen Operationen durchgeführt wird. Es erfolgt das Freipräparieren des Spatium pararektale und die subtile Darstellung des Ligaments, das entsprechend durchstochen wird. Diese Operation stellt ein hochspezialisiertes, technisch schwieriges Verfahren dar, das entsprechend bewertet werden muss.

Auch die abdominale Fixation des Scheidenblindsackes am Promontorium ggf. unter Benutzung eines Interponats aus Faszienstreifen oder alloplastischem Material, stellt ein eigenständiges Operationsverfahren dar, das technisch anspruchsvoll ist.

Die Ziffer ist bei entsprechender Durchführung der Leistung neben anderen Operationsschritten berechenbar.

\section{Kommentar zur Ziffer 3231 analog GOÄ}

Im Bereich der hinteren Scheidenwand wurde früher praktisch nur introitusnah operiert, indem eine Rektozele versenkt und der Beckenboden neu aufgebaut wurde. Die Bauchhöhle wird bei diesem Operationsverfahren nicht eröffnet. Die erweiterte Präparation im oberen Scheidenbereich wird in der GOÄ nicht abgebildet. Besteht nämlich neben der Rektozele eine größere Enterozele, bedarf die Korrektur dieses Bruchs einer eigenständigen Operationsleistung mit Eröffnung der Bauchhöhle. Es gibt dafür in der GOÄ keine adäquate Ziffer, so dass die Abrechnung über eine Analogziffer gemäß §6 GOÄ zwingend geboten ist. Die Ziffer ist bei entsprechender Durchführung der Leistung neben anderen Operationsschritten berechenbar.

Kommentar zur Ziffer 3283 analog für paravaginale Scheidenplastik (Paravaginal repair) oder andere Scheidenfixationen,

\section{z.B. Kuldoplastik nach McCall}

Die modernen Erkenntnisse über die Ätiologie des Deszensus haben gezeigt, dass bei Zelenbildung der vorderen Vaginalwand häufig kein medialer, sondern ein lateraler Defekt der endopelvinen Faszie bzw. ihrer seitlichen Verankerung an der Beckenwand besteht. Gelegentlich bestehen mediale und laterale Defekte gleichzeitig. Für die lateralen Defekte wurden in den letzten Jahren vaginale und abdominale Operationsverfahren entwickelt, die nicht durch die vorhandenen Ziffern der GOÄ abgebildet wer- 
den. Daher ist eine Abrechnung über eine Analogziffer gemäß § 6 GOÄ zwingend geboten. Die Ziffer ist bei entsprechender Durchführung der Leistung neben anderen Operationsschritten, insbesondere auch neben den Ziffern 1125 bzw. 1127 GOÄ, berechenbar.

Bei sehr mobiler Scheide kann neben der Hysterektomie oder isoliert zur Fixierung des Scheidenstumpfes eine Fixierung der Scheide an den Sacrouterinligamenten erfolgen, die entsprechend freipräpariert und dargestellt werden müssen. Auch dabei handelt es sich um einen eigenständigen Operationsschritt mit individueller Indikationsstellung.

\section{Urogynäkologie}

\section{Inkontinenz/Deszensus bei Uterus in situ}

1. Zentraler Defekt mit erheblichem Deszensus des inneren Genitals ohne ausgeprägte Zysto- und Rektozele (also kranialer und nicht kaudaler Defekt)

Jüngere Frauen, die noch kohabitieren bzw. noch prospektiv Kinderwunsch haben

- Möglichkeit 1:

Abdominales Vorgehen: Suprazervikale Hysterektomie, dann Kolposakropexie/Zervikosakropexie mit Fixation durch Band-Interponat

- Möglichkeit 2:

Erhalt des inneren Genitals und Ermöglichung einer Schwangerschaft: Keine suprazervikale Hysterektomie, aber Fixation von hinterem Vaginalgewölbe und Zervixhinterwand im Sinne der o.g. Sakro-Kolpo/Zerviko-Pexie (Tab. 3)

Tab. 3

\begin{tabular}{|c|c|c|c|}
\hline Ziffer & Leistungstext & Punkte & Euro \\
\hline \multicolumn{4}{|c|}{ Möglichkeit 1: } \\
\hline $1147 \mathrm{~A}$ & $\begin{array}{l}\text { Kolposakro-Zervikosakropexie mit } \\
\text { Fixation durch Interponat entspricht } \\
\text { Antefixierende Operation des Uterus } \\
\text { mit Eröffnung der Bauchhöhle, § } 6\end{array}$ & 1480 & 86,27 \\
\hline \multicolumn{4}{|c|}{ Möglichkeit 2: } \\
\hline 1147 & $\begin{array}{l}\text { Antefixierende Operation des Uterus } \\
\text { mit Eröffnung der Bauchhöhle - } \\
\text { Fixation vom hinteren Vaginalgewölbe }\end{array}$ & 1480 & 86,27 \\
\hline 1147 & $\begin{array}{l}\text { Antefixierende Operation des Uterus } \\
\text { mit Eröffnung der Bauchhöhle - } \\
\text { Fixation der Zervixhinterwand }\end{array}$ & 1480 & 86,27 \\
\hline
\end{tabular}

2. Ausladen des Douglas- und Gefahr der sekundären Deszensusproblematik im Rahmen der abdominalen Hysterektomie - Möglichkeit 1:

Geringgradigere Ausprägung: Abdominale Vaginae-Fixatio

- Fixation des Vaginalstumpfes an den Parametrien
- Möglichkeit 2:

Ausgeprägtere Problematik mit Neigung zu dorsalem Defekt: Abdominale Fixatio nach McCall $($ McCall = Fixation des Scheidenstumpfes an den verkürzten Uterosakralligamenten) (Tab. 4)

Tab. 4

\begin{tabular}{|c|c|c|c|}
\hline Ziffer & Leistungstext & Punkte & Euro \\
\hline \multicolumn{4}{|c|}{ Möglichkeit 1: } \\
\hline $\begin{array}{l}3283 \\
A^{*}\end{array}$ & $\begin{array}{l}\text { Abdominale Vaginae-Fixatio - Fixation } \\
\text { des Vaginalstumpfes an den Parametrien } \\
\text { entspricht Operation eines Nabel- } \\
\text { oder Mittellinien- oder Bauchnarben- } \\
\text { bruches, § } 6\end{array}$ & 1110 & 64,70 \\
\hline \multicolumn{4}{|c|}{ Möglichkeit 2: } \\
\hline $\begin{array}{l}3284 \\
A^{* *}\end{array}$ & $\begin{array}{l}\text { Abdominale Fixatio nach McCall, } \\
\text { Fixation des Scheidenstumpfes an den } \\
\text { verkürzten Uterosakralligamenten ent- } \\
\text { spricht Operation eines Nabel- oder } \\
\text { Mittellinien- oder Bauchnarbenbruches } \\
\text { mit Muskel- und Faszienverschiebe- } \\
\text { plastik, §6 }\end{array}$ & 2500 & 145,72 \\
\hline
\end{tabular}

* Baucheröffnung ist in der Ziffer 3283 A nicht enthalten

***Baucheröffnung ist in der Ziffer $3284 \mathrm{~A}$ enthalten

3. Inkontinenz und Deszensus, vor allem mit kranialen anterioren Defekt und Zystozele

- Möglichkeit 1:

Beginnende Symptomatik/prophylaktisch:

Abdominale Kolposuspension nach Hirsch (jeweils 2 Obturatorius-Faszien-Suspensionsnähte)

- Möglichkeit 2:

Ausgeprägtere Problematik:

Abdominale Kolposuspension nach Cowan (Einmalnaht nach Burch, einmal Obturatorius-Fasziennaht)

- Möglichkeit 3:

Zusätzlicher lateraler vaginaler Defekt/Verlagerungzystozele:

Abdominale Paravaginale Kolpopexie (zusätzlich zu Cowan) mit zusätzlichen 1 - 3 Nähten zu beiden Cowan-Nähten

- Möglichkeit 4:

TVT und vaginale paravaginale Kolpopexie (Tab. 5) 
Tab. 5

\begin{tabular}{|c|c|c|c|}
\hline Ziffer & Leistungstext & Punkte & Euro \\
\hline \multicolumn{4}{|c|}{ Möglichkeit 1: } \\
\hline $1780^{*}$ & $\begin{array}{l}\text { Plastische Operation zur Behebung der } \\
\text { Harninkontinenz }\end{array}$ & 1850 & 107,83 \\
\hline \multicolumn{4}{|c|}{ Möglichkeit 2: } \\
\hline $1780^{* *}$ & $\begin{array}{l}\text { Plastische Operation zur Behebung der } \\
\text { Harninkontinenz }\end{array}$ & 1850 & 107,83 \\
\hline \multicolumn{4}{|c|}{ Möglichkeit 3: } \\
\hline $\begin{array}{l}1780 \\
* * *\end{array}$ & $\begin{array}{l}\text { Plastische Operation zur Behebung der } \\
\text { Harninkontinenz }\end{array}$ & 1850 & 107,83 \\
\hline $\begin{array}{l}3283 \mathrm{~A} \\
* * * *\end{array}$ & $\begin{array}{l}\text { Abdominale paravaginale Kolpopexie } \\
\text { entspricht Operation eines Nabel- } \\
\text { oder Mittellinien- oder Bauchnarben- } \\
\text { bruches, § } 6\end{array}$ & 1110 & 64,70 \\
\hline \multicolumn{4}{|c|}{ Möglichkeit 4: } \\
\hline $\begin{array}{l}1781 \mathrm{~A} \\
* * * * *\end{array}$ & $\begin{array}{l}\text { TVT entspricht Operative Behebung der } \\
\text { Harninkontinenz mittels Implantation } \\
\text { eines künstlichen Schließmuskels, § } 6\end{array}$ & 2770 & 161,46 \\
\hline $\begin{array}{l}3231 \mathrm{~A} \\
* * * * * *\end{array}$ & $\begin{array}{l}\text { Vaginale paravaginale Kolpopexie } \\
\text { entspricht Operation des Mastdarm- } \\
\text { vorfalles bei Zugang vom After aus } \\
\text { oder perineal, } § 6\end{array}$ & 1150 & 67,03 \\
\hline
\end{tabular}

* $\quad$ Baucheröffnung ist in der Ziffer 1780 nicht enthalten

** Baucheröffnung ist in der Ziffer 1780 nicht enthalten

*** Baucheröffnung ist in der Ziffer 1780 nicht enthalten

**** Baucheröffnung ist in der Ziffer 3283 A nicht enthalten

***** Baucheröffnung ist in der Ziffer 1781 nicht enthalten

****** Baucheröffnung ist in der Ziffer 3231 A nicht enthalten

4. Inkontinenz und Deszensus mit zusätzlich hinterem Beckenbodendefekt (mit Rektozele)

- Möglichkeit 1:

Wie unter 3., aber zusätzlich hinterer Kolporrhaphie, Levatorplastik, ggf. Perinealrekonstruktion

- Möglichkeit 2:

Wie unter 3., aber noch ausgeprägterer hinterer Beckenbodendefekt: Benutzung des transvaginal durchgezogenen Vaginae fixatio-Fadens nach McCall zur vaginalen Rekonstruktion des hinteren Vaginalgewölbes. In schweren Fällen: vaginale sakrospinale Fixation nach Amreich-Richter (Tab. 6)

5. Trotz abdominaler Kolposuspension mit paravaginaler Fixation noch kaudale Zystozele/Urethrozystozele

Zusätzliche vaginale vordere Kolporrhaphie (Tab. 7)

6. Rezidiv-Zystozele/Rektozele

Ggf. Netzeinlage kombiniert mit Vorderer/Hinterer Kolporrhaphie (Tab. 8)
Tab. 6

\begin{tabular}{|c|c|c|c|}
\hline Ziffer & Leistungstext & Punkte & Euro \\
\hline \multicolumn{4}{|c|}{ Möglichkeit 1: } \\
\hline & wie unter Punkt 3., und zusätzlich & & \\
\hline 1126 & $\begin{array}{l}\text { Hintere Scheidenplastik mit Becken- } \\
\text { bodenplastik }\end{array}$ & 1290 & 75,19 \\
\hline \multicolumn{4}{|c|}{ Möglichkeit 2: } \\
\hline & wie unter Punkt 3., und zusätzlich & & \\
\hline 1126 & $\begin{array}{l}\text { Hintere Scheidenplastik mit Becken- } \\
\text { bodenplastik }\end{array}$ & 1290 & 75,19 \\
\hline \multicolumn{4}{|c|}{ Möglichkeit 2: Schwerer Fall } \\
\hline & wie unter Punkt 3., und zusätzlich & & \\
\hline 1126 & $\begin{array}{l}\text { Hintere Scheidenplastik mit Becken- } \\
\text { bodenplastik }\end{array}$ & 1290 & 75,19 \\
\hline 1147 & $\begin{array}{l}\text { Vaginale sakrospinale Fixation nach } \\
\text { Amreich Richter }\end{array}$ & 1480 & 86,27 \\
\hline
\end{tabular}

Tab. 7

\begin{tabular}{lllc}
\hline Ziffer & Leistungstext & Punkte & Euro \\
\hline 1125 & $\begin{array}{l}\text { Vordere Scheidenplastik } \\
\text { oder }\end{array}$ & 0924 & 53,86 \\
\hline 1127 & $\begin{array}{l}\text { Vordere und hintere Scheidenplastik mit } \\
\text { Beckenbodenplastik }\end{array}$ & 1660 & 96,76 \\
\hline
\end{tabular}

Tab. 8

\begin{tabular}{clcc}
\hline Ziffer & Leistungstext & Punkte & Euro \\
\hline 1127 & $\begin{array}{l}\text { Vordere und hintere Scheidenplastik mit } \\
\text { Beckenbodenplastik }\end{array}$ & 1660 & 96,76 \\
\hline 1781 A & $\begin{array}{l}\text { analog: Behebung der Rezidiv-Zystozele/ } \\
\text { Rektozele mit Netzeinlage entspricht } \\
\text { Operative Behandlung der Harninkonti- } \\
\text { nenz mittels Implantation eines künstli- } \\
\text { chen Schließmuskels, §6 }\end{array}$ & 161,46 \\
\hline
\end{tabular}


II. Inkontinenz/Deszensus nach Hysterektomie

1. Kranialer anteriorer Defekt

Abdominale Kolposakropexie (s. o.) (Tab. 9)

2. Kaudaler anteriorer Defekt

Vordere Kolporrhaphie/Rektokolporrhaphie mittels zusätzlicher supportiver Bandeinlage, z. B. TVT (Tab. 10)

3. Dorsaler Defekt

Hintere Kolporrhaphie/Rekolporrhaphie (Tab. 11)

4. Subtotalprolaps hintere Vaginalwand

- Möglichkeit 1:

Ausgedehnte hintere Kolporrhaphie mit Levatorplastik

- Möglichkeit 2:

Ausgedehnterer Defekt Hintere Kolporrhaphie/Rekolporrhaphie mit vaginaler sakrospinaler Fixation nach Amreich-Richter (einseitig bzw. bei breitem Defekt doppelseitig) (Tab. 12)

5. zusätzlich Analprolaps

Zusätzliches chirurgisches Vorgehen nach Rektomanometrie

6. Zusätzlicher Sphinkterdefekt

Zusätzliche chirurgische Maßnahme

Tab. 9

\begin{tabular}{|clcc}
\hline Ziffer & Leistungstext & Punkte & Euro \\
\hline 1147 A & $\begin{array}{l}\text { Kolposakro-Zervikosakropexie mit } \\
\text { Fixation durch Interponat entspricht }\end{array}$ & 1480 & 86,27 \\
& $\begin{array}{l}\text { Antefixierende Operation des Uterus } \\
\text { mit Eröffnung der Bauchhöhle, §6 }\end{array}$ & \\
\hline
\end{tabular}

Tab. 10

\begin{tabular}{|c|c|c|c|}
\hline Ziffer & Leistungstext & Punkte & Euro \\
\hline 1127 & $\begin{array}{l}\text { Vordere und hintere Scheidenplastik } \\
\text { mit Beckenbodenplastik }\end{array}$ & 1660 & 96,76 \\
\hline $\begin{array}{l}1781 \mathrm{~A} \\
*\end{array}$ & $\begin{array}{l}\text { TVT entspricht Operative Behebung der } \\
\text { Harninkontinenz mittels Implantation } \\
\text { eines künstlichen Schließmuskels, } \S 6\end{array}$ & 2770 & 161,46 \\
\hline
\end{tabular}

Tab. 11

\begin{tabular}{|c|c|c|c|}
\hline Ziffer & Leistungstext & Punkte & Euro \\
\hline 1127 & $\begin{array}{l}\text { Vordere und hintere Scheidenplastik } \\
\text { mit Beckenbodenplastik }\end{array}$ & 1660 & 96,76 \\
\hline
\end{tabular}

Tab. 12

\begin{tabular}{|c|c|c|c|}
\hline Ziffer & Leistungstext & Punkte & Euro \\
\hline \multicolumn{4}{|c|}{ Möglichkeit 1: } \\
\hline 1126 & $\begin{array}{l}\text { Hintere Scheidenplastik mit } \\
\text { Beckenbodenplastik }\end{array}$ & 1290 & 75,19 \\
\hline \multicolumn{4}{|c|}{ Möglichkeit 2: } \\
\hline 1126 & $\begin{array}{l}\text { Hintere Scheidenplastik mit } \\
\text { Beckenbodenplastik }\end{array}$ & 1290 & 75,19 \\
\hline 1147 & $\begin{array}{l}\text { Vaginale sakrospinale Fixation } \\
\text { nach Amreich Richter }\end{array}$ & 1480 & 86,27 \\
\hline
\end{tabular}

\section{Endoskopische Operationsverfahren („minimal access“)}

\section{Vorbemerkung und Übersicht}

Gerade im Bereich der minimalinvasiven Operationsverfahren ist die operative Gynäkologie in den letzten Jahren einem erheblichen Strukturwandel unterworfen worden. Dies basiert einerseits auf der Einführung neuer videoskopischer, endoskopischer Verfahren, andererseits der Einführung subtiler, teils thermischer Operationsverfahren.

In jüngster Zeit hat mit ungeahnter Geschwindigkeit die operative Endoskopie bzw. die videoendoskopische Chirurgie in fast allen operativen Disziplinen, wie auch in der Gynäkologie, Fuß gefasst. Gerade in der Gynäkologie ist das Feld für die endoskopischen Operationsverfahren besonders groß, da die operative Endoskopie hier auf mehreren Säulen ruht (Zentralblatt 117).

Die Gesamtheit der operativ-endoskopischen Verfahren per Videoskopie wird als „minimal-operative Operationsverfahren“ bezeichnet, wobei die jeweilige Säule dem endoskopischen Zugangsweg entspricht:
1. Laparoskopie
2. Hysteroskopie
3. Falloposkopie
4. Kolposkopie
5. Retziusskopie
6. Axilloskopie
7. Sentinel Lymphnode Detektion

Bei der detaillierten Darstellung im Folgenden wird einerseits auf die Standardoperationsverfahren per minimalinvasivem Zugang, andererseits auf neue, bisher noch nicht verfügbare Operationsverfahren fokussiert und eine schematische Darstellung der neuen Nomenklatur erstellt.

\section{Laparoskopie}

1.1 Diagnostische Laparoskopie, ggf. mit

- Chromopertubation

- Spülzytologie

- Bakteriologie

- Probeexzision 


\subsection{Endometrioseoperationen}

Hier profitieren leichtere Endometriosefälle allein schon von der endoskopischen Diagnostik, da sie so einer konsequenten Therapie zugeführt werden können. Eine Endometriose nach dem AFSScore I und II ist in den meisten Fällen laparoskopisch angehbar.

Sehr leicht wird aber auch, vergleichbar zur laparoskopischen Adhäsiolyse, die Grenze des endoskopisch Sinnvollen überschritten und eine komplexe Endometriose, z. B. mit Befall der Ovarien oder des Septum rectovaginale, endoskopisch nur insuffizient therapiert und damit die Rezidivrate noch erhöht. Häufig ist die Methode bei fortgeschrittener Endometriose überfordert.

\subsubsection{Elektrochirurgische Laser-Endometrioseresektionen bzw. Koagulationen}

\subsubsection{Endometriomexstirpation im Bereich inneres Genital und Peritoneum}

\subsection{Tuboplastische Operationen bei distaler und proximaler Tubenpathologie bzw. Refertilisierungen}

Obwohl die Rekonstruktion distaler Tubenpathologie als weit verbreitete Indikation zum laparoskopisch-rekonstruktiven Eingriff gilt, ist gerade diese Indikation sehr kritisch zu sehen. Hier sollte sich der Operateur bewusst sein, dass ein tubenrekonstruktiver Eingriff nur einmal vorgenommen werden kann, da eine nicht optimal durchgeführte Fimbriolyse, Fimbrioplastik oder Salpingostomie einen irreparablen Tubenschaden darstellt.

Darüber hinaus kann nur ein spezialisiertes Zentrum entscheiden, ob der Eingriff nicht eher mikrochirurgisch erfolgen sollte bzw. eine Tubenrekonstruktion, beispielsweise bei dickwandiger oder gekammerter Sactosalpinx, eine unnötige Belastung für die Patientin darstellt und eine In-vitro-Fertilisation a priori bessere Erfolgsaussichten hätte.

\subsubsection{Salpingolyse}

\subsubsection{Ovariolyse}

\subsubsection{Salpingostomie}

\subsubsection{Fimbrioplastik}

\subsubsection{Segmentresektion mit End-zu-End-Anastomosierung}

\subsubsection{Refertilisierung}

\subsection{Ovarialtumoren}

Vor dem Hintergrund der Komplexität der Pathologie zystischer Adnextumoren kann keine „100\%ig sichere“ Selektion hinsichtlich des endoskopischen Zuganges bei „vermutet“ benignen Ovarialtumoren erfolgen.

Daher sollte im Zweifelsfalle der endoskopische Zugang trotz optimaler Selektion nur bei der Möglichkeit der intraoperativen Schnellschnittdiagnostik und des jederzeitigen Umschaltens auf eine Staging-Laparotomie gewählt werden. Die suffiziente Aufklärung der Patientin ist selbstverständlich.
Auch sollte in diesen Fällen die laparoskopische Entfernung der Tumoren bzw. Adnexen möglichst im Bergesack erfolgen, wobei dies auch nur eine „trügerische“ Sicherheit darstellen kann, da zur Zeit nicht alle auf dem Markt befindlichen Bergesäcke die gleiche Qualität aufweisen und das sachgerechte Handling des Bergesackes übungsbedürftig ist.

1.4.1 Zystexstirpationen, Zystektomie, Zystexstirpation, Zystfenistration, Zystenpunktion

\subsubsection{Partielle Ovarektomie}

1.4.3 Elektrochirurgische bzw. Laserstichelungen multipler Zysten bei PCO-Syndrom

\subsubsection{Tumorexstirpationen}

\subsubsection{Elektronische Morcellierung über subkutane Minilaparotomie und Bergung}

\subsubsection{Transperitoneale Bergung über Bergesack}

\subsubsection{Adnexektomie}

\subsubsection{Ovarektomie}

\subsubsection{Bergung}

Die endoskopische Ovarialchirurgie breitet sich aktuell vielerorts ebenso rasant wie kritiklos aus. Dabei werden die technischen Möglichkeiten sowohl der organerhaltenden wie der ablativen endoskopischen Ovarialchirurgie so kontrovers diskutiert, dass eine Strukturierung von Indikation zum endoskopischen Vorgehen ebenso wie die optimale endoskopische Bergung von Ovarialtumor bzw. Adnexe oberste Priorität hat.

Das Risiko des endoskopischen „Anoperierens“ wird in der Literatur mit zwischen $0,4 \%$ und $3 \%$ angegeben.

Bei der klinischen Anwendung der Bergesäcke zeigt sich, dass eine optimale Bergung ein intensives Training erfordert. Als typische Probleme zeigen sich Volumendiskrepanzen zwischen Bergesack bzw. Bauchdeckeninzision und zu entfernendem Gewebe (in $28 \%$ der Fälle).

Anhand der Materialprüfungen konnten signifikante $(\mathrm{p}<0,05)$ Unterschiede in der Reißfestigkeit zwischen dem besten und dem schlechtesten Bergesack festgestellt werden. Naturgemäß kann vor dem Hintergrund der Komplexität der Pathologie zystischer Adnextumoren keine „100\%ig sichere“ Selektion hinsichtlich des endoskopischen Zuganges erfolgen.

Daher darf der endoskopische Zugang nur bei optimaler Selektion und nur bei der Möglichkeit der intraoperativen Gewebeschnittdiagnostik und des jederzeitigen Umschaltens auf eine Staging-Laparotomie gewählt werden.

Die suffiziente Aufklärung der Patientin ist selbstverständlich.

Bei postmenopausalen Patientinnen ist die ablative Therapie die Therapie der Wahl. 
Die laparoskopische Entfernung der Tumoren bzw. Adnexen sollte möglichst im Bergesack erfolgen. Keinesfalls sollte jedoch der laparoskopische Bergesack den Operateur in Sicherheit wiegen, da zur Zeit nicht alle auf dem Markt befindlichen Bergesäcke eine suffiziente Qualität aufweisen.

\subsection{Die operative Therapie der Tubargravidität}

Der Wandel im Erscheinungsbild der Tubargravidität

Auch heute stellt die Tubargravidität noch eine Herausforderung für den Operateur dar. Dabei ist die Problematik jedoch im Vergleich zu früheren Jahrzehnten oder gar dem ausklingenden vorigen Jahrhundert anders gelagert. Dies liegt zum einen im Wandel des Erscheinungsbildes der Tubargravidität begründet. Zum anderen tritt die mit der Tubargravidität assoziierte Sterilitätsproblematik mehr und mehr in den Vordergrund.

Weit bis in die erste Hälfte des Jahrhunderts stand die akute vitale Bedrohung der Patienten durch die Tubargravidität und damit die ablative Operation als vitale Maßnahme im Vordergrund. Erst um die Jahrhundertwende konnte die Mortalitätsrate, die noch weit über zwei Drittel der betroffenen Frauen betrug, schrittweise durch die Einführung ablativer Operationstechniken gesenkt werden.

Aufgrund moderner diagnostsicher Verfahren, wie der $\beta$-HCGVerlaufskontrolle und der transvaginalen sonographischen Untersuchung ist heute ein völliger Wandel im Erscheinungsbild der Tubargravidität eingetreten. Der überwiegende Teil der Patientinnen kann aufgrund frühester Diagnostik der Extrauteringravidität einer geplanten und somit nicht notfallmäßigen Operation zugeführt werden. In etwa einem Drittel aller Fälle handelt es sich sogar um eine stehende Tubargravidität, bei der es nicht zur Tubenwandruptur oder zum Austritt von Blut in die freie Bauchhöhle gekommen ist.

Ein weiterer wichtiger Aspekt der Extrauteringravidität ist deren sozio-ökonomische Bedeutung, die mehr und mehr in den Mittelpunkt des Interesses tritt.

So hat sich die Inzidenz der Extrauteringravidität im letzten Jahrzehnt verdreifacht, ohne dass eine Klärung der Ätiopathogenese definitiv erreicht werden konnte.

Vor diesem Hintergrund wird die Bedeutung der mit der Tubargravidität assoziierten Sterilitätsprobleme erst in ihrer ganzen Tragweite deutlich. So muss nach radikaler operativer Therapie der Tubargravidität in bis zu 70\% der Fälle, beim Auftreten der Tubargravidität in der ersten Schwangerschaft sogar in bis zu 90\% der Fälle, mit einer sekundären tubaren Sterilität auch bei kontralateral vorhandener Tube gerechnet werden.

Damit stellt die frühestmögliche Diagnose und darüber hinaus die Wahl des individuell-spezifischen Operationsverfahrens eine klinisch-wissenschaftliche Herausforderung dar.

\section{Die Operationstechniken}

- Ablative Verfahren

Radikale Operationsverfahren, wie die totale oder partielle Salpingektomie oder die Adnexektomie, wurden schon vor über 100 Jahren beschrieben.

\section{- Organerhaltende Verfahren}

Für die organerhaltende, konservative oder besser konservierende Behandlung der Tubargravidität stehen verschiedene mikrochirurgische und/oder laparoskopische Operationsverfahren zur Verfügung. Prinzipiell muss dabei zwischen kontiuitätserhaltenden und kontinuitätsunterbrechenden Techniken unterschieden werden.

Indirekt organerhaltende (kontinuitätsunterbrechende)
Techniken

\subsubsection{Einfache Segmentresektion}

Bei der einfachen Segmentresektion handelt es sich um die Resektion des Tubensegmentes, das das Schwangerschaftsprodukt trägt, ohne dass eine primäre End-zu-End-Anastomosierung der Tubenstümpfe erfolgt.

Die Resektion erfolgt unter Erhalt der Mesosalpinx. Die Tubenstümpfe werden durch Peritonealisierung versenkt, um über die Möglichkeit der zweizeitigen tubotubaren End-zu-End-Anastomosierung zu verfügen.

Zur Segmentresektion wird zunächst die Tube proximal und peripher der Tubargravidität koaguliert.

Aufgrund der Tatsache, dass die Tubenstümpfe unter Umständen später einer Anastomosierung zugeführt werden müssen, steht das atraumatische Handling der Tube, möglichst mit mikrochirurgischem Instrumentarium, außer Frage. Nun erfolgt die Resektion scharf oder mit der Mikroelektrode. Die Tubenstümpfe werden anschließend durch Peritonealisierung mit resorbierbaren Fäden (Vicryl 6-0) versenkt.

Die Vorteile der Segmentresektion bestehen einerseits darin, dass das - zumindest hypothetisch - geschädigte Tubensegment entfernt ist und damit ein Rezidiv nicht mehr infrage kommt. Darüber hinaus ist das Risiko, Trophoblastreste zurückzulassen, ausgeschlossen, da eine Trophoblastinfiltration bis in die Muscularis bzw. bis in die Mesosalpinx hinein nie ausgeschlossen werden kann.

Aufgrund dieser Vorteile empfiehlt sich die Segmentresektion bei Patientinnen, bei denen eine Tubenkontinuität nicht oder nicht in jedem Falle wiederhergestellt werden muss. Die Möglichkeit einer zweizeitigen Rekonstruktion ist bei einer verbleibenden Tubenrestlänge von mindestens $4 \mathrm{~cm}$ in jedem Fall möglich.

Die Gewebeverhältnisse für eine primäre Anastomosierung sind in den meisten Fällen durch ödematöse Gewebsveränderungen oder intramurale Hämatombildungen ungünstig.

\subsubsection{Segmentkoagulation}

Der Vollständigkeit halber soll die technisch sehr einfache Zerstörung der Tubargravidität durch eine Elektrokoagulation erwähnt werden. Die Segmentkoagulation ist nur bei extrem kleinen Tubargraviditäten möglich. Darüber hinaus ist hier in keiner Weise eine histologische Sicherung gegeben. Um in diesen Fällen eine größtmögliche Sicherheit, was die Zerstörung der gesamten Trophoblastzellen angeht, zu erzielen, ist eine ausgedehnte Elek- 
trokoagulation notwendig. Eine Schädigung bis weit in die Mesosalpinx ist in keinem Falle auszuschließen.

\section{- Direkt organerhaltende (kontinuitätserhaltende) Techniken}

\subsubsection{Segmentresektion mit einzeitiger End-zu-End- Anastomosierung}

Das einzeitige Vorgehen zur primären End-zu-End-Anastomosierung nach Segmentresektion ist den Fällen vorbehalten, bei denen die kontralaterale Tube fehlt oder einen schweren Tubenschaden aufweist. Die prinzipielle Voraussetzung ist dabei jedoch, dass für eine primäre mikrochirurgische Intervention günstige Voraussetzungen vorhanden sind. Zum einen sollen die anatomischen Gegebenheiten entsprechend sein und die Tubargravidität nicht zu ausgeprägten Tubenschäden, wie einer Wandinfarzierung oder einem intramuralen oder in die Mesosalpinx entwickelten Hämatom geführt haben. Nicht zu vernachlässigen ist zum anderen auch die Tatsache, dass zu dieser aufwendigen Operation ein mikrochirurgisches Team einsetzbar sein muss. Die Technik der End-zu-End-Anastomosierung entspricht der bekannten mikrochirurgischen Anastomosetechnik.

Ein weiteres ungeklärtes Problem ist - und diese Frage bezieht sich vor allem auf die Segmentresektion - ob das zweizeitige dem einzeitigen Vorgehen vorzuziehen ist.

Eine primäre Rekonstruktion ist nur in jenen sehr seltenen Fällen zu befürworten, bei denen die Tubargravidität sehr früh diagnostiziert wurde, sich im Isthmus tubae oder im isthmoampullären Teil befindet und nach Resektion des graviden Tubenteils keine zu große Lumendifferenz besteht. Vor allem aber sollte das Gewebe nicht zu ödematös und in den Gewebsschichten kein Hämatom vorhanden sein, ebenso keine entzündliche Reaktion. Die verbleibende Tubenlänge sollte mindestens $4-5 \mathrm{~cm}$ betragen.

Da jedoch die Gewebsverhältnisse in den meisten Fällen für eine Anastomose sehr ungünstig sind, wird heute allgemein das zweizeitige Vorgehen befürwortet. Hierbei beschränkt man sich zunächst auf die Segmentresektion, um erst später, falls über die kontralaterale Seite keine intrauterine Gravidität oder ein EU-Rezidiv eintritt, die Anastomose im reizlosen Gewebe durchführen zu können. Aufgrund der kleinen Fallzahlen liegen über diese wichtige Frage bisher keine wegweisenden Ergebnisse vor. Dies gilt auch für die Frage, ob ein pathologischer Tubenbefund der kontralteralen Seite in gleicher Sitzung durch eine rekonstruktive makro- bzw. mikrochirurgische Technik angegangen werden soll. Unseres Erachtens haben hier die gleichen Argumente wie bei der Frage der einzeitigen oder zweizeitigen Anastomose Gültigkeit.

\subsubsection{Lineare Salpingotomie}

Hier ist die lineare Salpingotomie, das Aufschneiden der Tubenwand und das Herauspräparieren der Tubargravidität gemeint. Dies ist eines der aufwendigsten Verfahren, da nach Exstirpation der Tubargravidität gleichzeitig eine plastische Rekonstruktion der Tubenwand notwendig ist, wiederum oft wesentlich schwieriger ist und hinsichtlich einer späteren Schwangerschaft subtiler durchgeführt werden muss als die Salpingotomie selbst.
In gleichem Maße, wie die Anzahl der in sehr frühem Stadium diagnostizierten Tubargraviditäten steigt, gewinnt die kontinuitätserhaltende Therapie der Tubargravidität durch eine lineare Salpingotomie an Bedeutung. Die Tubenwand wird dabei über dem Gestationsprodukt linear inzidiert und die Tubargravidität vorsichtig herausgelöst. Die Salpingotomie bietet sich an bei Frühfällen, d.h. bei nicht rupturierter Tubenwand und noch fehlender Hämatombildung, besonders bei isthmo-ampullärem oder ampullärem Sitz. Dazu wird nach atraumatischer Fixierung der Tube die lineare Salpingotomie antimesenterial oder, in Abhängigkeit von der anatomischen Situation, mesenterial durchgeführt.

Bei Beachtung der einzuhaltenden möglichst atraumatischen Kautelen und der möglichst sorgfältigen Trophoblastausräumung ist die lineare Salpingotomie, bis auf wenige Fälle, bei denen es zu stärkeren Blutungen aus dem Bett der Tubargravidität kommen kann, eine sehr einfache Operationstechnik, die ohne mikrochirurgische Instrumente, insbesondere ohne Sicht durch das Operationsmikroskop durchführbar ist.

Im Hinblick auf die anzuwendende Präparationstechnik zur Salpingotomie wird auf den Vergleich der verschiedenen Techniken am Ende des Beitrags verwiesen.

Nach sorgfältiger Blutstillung im Bereich des Wundbettes der Tubargravidität erfolgt die Toilette des Intraperitonealraumes. Von einigen Arbeitsgruppen wird die Salpingotomiewunde mittels Einzelknopfnähten oder einer fortlaufenden Seromuskularnaht verschlossen. Immer mehr Arbeitsgruppen verzichten jedoch bei einer Salpingotomie, die nicht mehr als $2-3 \mathrm{~cm}$ beträgt, auf einen Wundverschluss. In eigenen tierexperimentell-chirurgischen Untersuchungen an den Tuben von Minischweinen konnte gezeigt werden, dass nach linearer Salpingotomie in fast allen Fällen eine Abheilung ohne Dehiszenz oder Fistelbildung resultiert.

1.5.5 Transampulläre Expression der Tubargravidität („milk-out“) Die einfache Expression oder „milk-out“-Technik beruht auf dem Phänomen des spontanen transampullären Tubarabortes aus dem äußere Teil der Ampulle oder dem Fimbrienbereich. Das Exprimieren des Tubarabortes erfolgt vorsichtig in Richtung auf das Fimbrienende zu. Dabei besteht das Problem, das Trophoblastgewebe gänzlich entfernen oder eine intraampulläre Blutstillung bei diffuser Blutung erreichen zu können. Als alternative Technik ist hier die periphere transampulläre lineare Salpingotomie vorzuziehen. Hier ist eine wirkliche Exploration sowie eine exaktere Blutstillung möglich.

Im Gegensatz zur distalen ampullären Salpingotomie werden beim Exprimieren des Tubarabortes häufiger Rezidive beschrieben. Nach Timonen ist zudem die Schwangerschaftsrate bei der invasiven Therapie höher als beim einfachen „milk-out“. Wie bei der isthmischen oder isthmo-ampullären Tubargravidität führen wir auch in Fällen einer fimbriennahen oder ampullären linearen Salpingotomie keinen Nahtverschluss durch.

\subsubsection{Organerhaltende laparoskopische Verfahren}

Mit der Optimierung operativ-laparoskopischer Verfahren tritt die Behandlung der Tubargravidität per laparotomiam mehr 
und mehr in den Hintergrund und bleibt bestimmten Indikationsbereichen vorbehalten. Denn sowohl ablative Verfahren als auch die bereits dargestellten indirekt oder direkt tubenerhaltenden Verfahren können laparoskopisch durchgeführt werden.

\section{- Technische Durchführung}

Die laparoskopischen Techniken unterscheiden sich durch die Wahl der ablativen bzw. der direkt oder indirekt organerhaltenden Präparationstechniken.

In jedem Falle werden suprasymphysär im kranialen Schamhaarbereich drei Hilfseinstiche angelegt, wobei der erste Einstich in der Medianlinie, die beiden weiteren Einstiche lateral des Verlaufs der Arteria epigastrica angelegt werden. In allen Fällen wird über die lateralen Einstiche die Tube über atraumatische Fasszangen dargestellt. Über den mittleren Hilfseinstich bzw. über den Arbeitskanal des Laparoskops werden Schere, Bipolarzange, Endokoagulator oder andere Applikatoren eingeführt. Bei der Anwendung der Endoschlinge nach Roeder wird über den mittleren Einstich der Applikator für die Endoligatur intraperitoneal eingeführt.

Zur Entfernung der Tubargravidität bzw. der exzidierten Tubenoder Adnexanteile wird durch den laparoskopischen Arbeitskanal oder bei größeren Gewebsanteilen nach Umdilatation des medianen Einstichs von einem 5-mm-Trokar auf einen 10-mmTrokar mit einem jeweils dem Durchmesser des Trokars oder Arbeitskanals angepassten Instrument das Gewebe aus dem Intraperitonealraum wird eine Toilette des Intraperitonealraums unter Entfernung aller Blutkoagel durchgeführt.

Die Dokumentation der kontralateralen Tubenverhältnisse, inkl. einer Chromopertubation, ist unabdingbar.

Wichtige Details der einzelnen laparoskopischen Präparationstechniken (partielle Salpingektomie, Segmentresektion, lineare Salpingotomie) sollen im folgenden dargestellt werden.

\section{- (Partielle) Salpingektomie}

Die Salpingektomie bzw. partielle Salpingektomie erfolgt wie die Adnexektomie über die Schlingentechnik mittels Endoschlinge oder Elektrokoagulation. Die Endoschlingen werden nacheinander über den Applikator intraperitoneal eingeführt und über die abzutragenden Gewebeanteile gelegt. Nach Anziehen der vorgefertigten Schlinge sitzt der Knoten fest auf Tubenteil bzw. Adnexstiel. Die Elektrokoagulation ohne Schlinge ist zusätzlich ebenso etabliert wie die Schlingenapplikation.

\section{- Segmentresektion}

Die laparoskopische Segmentresektion wird analog der Segmentresektion per laparotomiam durchgeführt. Sie bietet sich als Alternative zur Salpingotomie gerade bei proximal lokalisierten Tubargraviditäten an, da hier das Risiko der sekundären Tubenstenosierung bzw. der Belassung von Trophoblastresiduen zu minimieren ist. Die laparoskopische Segmentresektion war erstmals von Sapphiro und Adler sowie von der Arbeitsgruppe von Gomel beschrieben worden.

Zur Vermeidung von Blutungen aus der Mesosalpinx kann das Tubensegment von der Mesosalpinx nach Bipolarkoagulation oder nach Setzen einer Endoschlinge ebenfalls völlig bluttrocken abgetrennt werden.

\section{- Lineare Salpingotomie}

Die laparoskopische Salpingotomie wird vorzugsweise mit der Elektrode und dem Operationslaser durchgeführt, da hier eine maximale Gewebeschonung durch atraumatische Präparation möglich ist.

In allen Fällen der laparoskopisch-operativen Intervention müssen eine Bipolarkoagulationseirnichtung und eine suffiziente Saug-Spül-Einrichtung vorhanden sein. Denn bei jeder laparoskopischen Operation kann es zu Blutungen kommen. Die Blutung auch aus einer kleineren Arterie, sei es aus dem Wundbett der Tubargravidität, sei es eine stärker spritzende Blutung beispielsweise aus dem Ramus tubarius, kann mit der Lasertechnik nicht koaguliert werden, so dass hier eine Bipolarkoagulation unter gleichzeitigem Saug-Spül-Einsatz vonnöten ist.

Folgende Präparationsverfahren, wie die scharfe Salpingotomie, die Laser-Salpingotomie, die Präparation mittels Schere oder mittels laparoskopischem Skalpell nach vorheriger Vasopressinapplikation stehen zur Verfügung. Hier wird eine Vasopressinlösung verwendet, die aus anästhesiologischen Gründen hochverdünnt werden muss (Por-8, wenige ml einer Lösung, bei der eine Por-8 Ampulle mit 2,5 I.E. auf $25 \mathrm{ml}$ verdünnt wird und davon $10 \mathrm{ml}$ aufgezogen werden).

Die Endokoagulationstechnik wird von uns nicht eingesetzt, da hier ein relativ breiter Gewebesaum koaguliert wird.

Gerade bei der laparoskopischen Salpingotomie muss bei der Herauspräparation der Tubargravidität sehr vorsichtig, möglichst stumpf präpariert werden, da hier die Gefahr der Belassung von Trophoblastgewebe am größten ist.

Wie bereits dargestellt, kann in den meisten Fällen auf einen Nahtverschluss der Salpingotomie verzichtet werden.

Die sorgfältige Blutstillung und Toilette des Intraabdominalraums steht wie bei jeder laparoskopischen Operation außer Frage.

\section{- Operativ-technisches Vorgehen und zusammenfassende Wertung}

In gleichem Maße wie die Zahl der unter Notfallbedingungen durchgeführten Operationen von Tubargraviditäten abgenommen hat, hat die Zahl der operativen Eingriffe bei Extrauteringraviditäten mit fertilitätserhaltender Indikation zugenommen.

Schon 1895 wurde von Broschownik in einem Satz die Problematik der konservativen Operation von Tubargraviditäten zusammengefasst: „Unser Ziel muss sein, unter Herabsetzung der Gefahren doch konservativ zu bleiben!“

Somit ist die Basis der konservierenden Operationstechniken, die Tube zu erhalten, wann immer von der Sache her vertretbar, von der Patientin gewünscht und von ärztlicher Seite machbar. Somit stehen für die organerhaltende Therapie die möglichst frühzeiti- 
ge Diagnose der Tubargravidität und die Selektion des Krankengutes für ein organerhaltendes Vorgehen im Vordergrund.

Die organerhaltende Operation ist in fast 95\% der Fälle möglich.

- Indikationen für ein organerhaltendes Procedere

Bei Patientinnen mit Kinderwunsch ist prinzipiell ein organerhaltendes operatives Verfahren der Tubargravidität indiziert. Ein organerhaltendes Vorgehen ist bei Patientinnen im gebärfähigen Alter auch bei nur prospektiv bestehendem Kinderwunsch ratsam. Allerdings sollten bei diesen Patientinnen die Vorteile eines kontinuitätserhaltenden Verfahrens und die damit verbundenen Risiken sorgfältig gegeneinander abgewogen werden.

Insgesamt ist heute gesichert, dass nach konservativer Behandlung einer Tubargravidität die Fertilität höher ist als nach ablativem Vorgehen. Dies trifft auch für Fälle mit kontralateral intakter Tube zu. Während vor einer Tubargravidität 20\% der Patientinnen eine Fertilitätsstörung aufwiesen, trat eine Fertilitätsproblematik nach einer Tubargravidität bei 50\% der Patientinnen auf.

\section{- Risiken}

In jedem Falle eines organerhaltenden Vorgehens müssen die damit verbundenen Risiken abgewogen und mit der Patientin besprochen werden. Dabei stehen typische Komplikationen der tubenerhaltenden Operationen, bei denen das Bett des Gestationsproduktes nicht komplett exzidiert wird, Nachblutungen sowie persistierende Tubargraviditäten im Sinne von Trophoblastresiduen im Vordergrund. Mit diesen Risiken ist in bis zu 5\% der Fälle zu rechnen.

Unabdingbar ist daher eine routinemäßige postoperative Kontrolle des Serum- $\beta$-hCG-Wertes bis zur Nachweisbarkeitsgrenze.

- Kontraindikationen für ein organerhaltendes Procedere

In keiner Weise ist eine kontralateral intakte Tube ein Grund für ein ablatives Vorgehen. Hier sollte lediglich die Entscheidung für einen kontinuitätserhaltenden oder einen kontinuitätsunterbrechenden Eingriff zu treffen sein.

- Das operativ-technische Vorgehen

Die Wahl des Operationsverfahrens, insbesondere des konservativen Operationsverfahrens, hängt, wie erwähnt, ab

- von dem aktuellen oder prospektiven Kinderwunsch der Patientin,

- dem Alter der Patientin,

- dem Status der kontralateralen Tube,

- der Lokalisation der Tubargravidität.

Insbesondere stehen bei den operativ-technischen Überlegungen die Forderungen nach der Minimierung des operativen Traumas, dem das endoskopische Vorgehen voll und ganz entspricht, im Vordergrund.

Voraussetzung für das laparoskopische Vorgehen ist die Kreislaufstabilität der Patientin. Dagegen ist der Nachweis von intraperitonealem Blut in keiner Weise eine Kontraindikation zur diagnostischen bzw. therapeutischen Laparoskopie. Das ist auch der Grund dafür, dass die Douglaspunktion zur Planung des ope- rativ-technischen Vorgehens vollständig an Bedeutung verloren hat.

Da beim operativ-laparoskopischen Vorgehen über eine lineare Salpingotomie die Gefahr von Nachblutungen bzw. die Gefahr des Verbleibens von Trophoblastresiduen besteht, sollte diese Methode nur den Patientinnen mit Kinderwunsch vorbehalten bleiben. Bei Patientinnen mit abgeschlossener Familienplanung dürfte das ablative Verfahren im Sinne einer partiellen oder totalen Salpingektomie die größte Sicherheit bieten.

Bei Patientinnen mit bestehendem Kinderwunsch und kontralateral verschlossener oder fehlender Tube ist entweder eine lineare Salpingotomie oder eine Segmentresektion per Laparotomie mit einzeitiger End-zu-End-Anastomosierung die Methode der Wahl.

\subsection{Uterusantefixation}

\subsection{Ovarialtranspositionen bzw. -suspensionen}

\subsection{Vaginalplastiken und Neovaginaanlage}

Die Möglichkeit der endoskopischen Durchführung der Neovaginaanlage, in erster Linie in der Modifikation des Vorgehens nach Vecchietti erspart den gerade häufig sehr jungen Patientinnen in den meisten Fällen die Laparotomie, wobei das Risiko der Blasenläsion beim endoskopischen Vorgehen nicht vernachlässigt werden darf.

\subsection{Ureterolyse}

Die Ureterolyse stellt nur dann eine separate Leistung dar, wenn eine komplette Freilegung der Beckenwand und Ureterolyse bei starken Beckenwandverwachsungen, insbesondere bei Endometriose, Adhäsionen bzw. retroperitonealer Fibrosierung, erforderlich wird; in diesem Fall ist eine übermäßig komplizierte Operation notwendig, da die endoskopische Freilegung der Beckenwände im Bereich der großen Gefäße zur Lösung des Ureters im Vergleich zum Eingriff an Tube, Ovar oder ganzer Adnexe in den meisten Fällen den schwierigen Eingriff mit höherer Komplikationsrate darstellt. Von daher handelt es sich nicht um einen separaten Eingriff. Falls die genannten Bedingungen nicht vorliegen, liegt auch keine separate Leistung vor.

\subsection{LUNA}

(Elektrochirurgische oder „Laserthermische Uterine Nervenablation“)

\subsection{LAVH}

Im Gegensatz zu dem noch nicht klar definierbaren Benefit der laparoskopischen bzw. endoskopisch-assistierten partiellen Hysterektomie ist der Vorteil bei der laparoskopisch-assistierten vaginalen Hysterektomie unbestritten (K. Neis). Gerade dieser endoskopische Eingriff zeigt beispielhaft die Wertigkeit des ergänzenden Einsatzes der Endoskopie im operativ-gynäkologischen Spektrum.

Dank des endoskopisch-assistierten Eingriffes könnte dem in vielen Ländern rückläufigen Trend des vaginalen Zuganges zur Hysterektomie, einer ureigenen Operationstechnik der operativen Gynäkologie, entgegengewirkt werden. 


\subsubsection{LAVH bei Korpuskarzininom in Kombination mit Lymphonodektomie}

1.11.3 Laparoskopisch assistierte vaginale partielle Hysterektomie (z.B. laparoskopisch-assistierte suprazervikale Hysterektomie). Im Gegensatz zu etablierten endoskopischen Verfahren sind viele neue Techniken Gegenstand derzeitiger Forschung. Diese Techniken können zum jetzigen Zeitpunkt und Stand der Forschung keineswegs allgemein empfohlen werden. Sie sollten bis zum Vorliegen ausreichender Erkenntnisse der kritischen Evaluierung spezialisierten Zentren vorbehalten bleiben.

Dies gilt in erster Linie für das Spektrum der laparoskopischen bzw. laparoskopisch-assistierten partiellen Hysterektomien (im Gegensatz zur laparoskopisch-assistierten vaginalen Hysterektomie) sowie für endoskopische Manipulationen im Bereich gynäkologischer Malignome.

Lässt man im Hinblick auf die „partielle Hysterektomie“ die operativen Schulen der Hysterektomie der letzten 100 Jahre Revue passieren, so drängt sich der Satz von Tadir auf: „Soll das Rad neu erfunden werden?“

Gerade hier scheint es eine Renaissance lange verlassener Operationstechniken, wie der suprazervikalen Hysterektomie, Anfang des Jahrhunderts vaginal durchgeführt, z. B. nach Cashman mit elektrischer Destruktion des zervikalen Endometriums, zu geben.

Bei dieser Technik ist ein Benefit für die Patientin im Vergleich zum rein vaginalen Vorgehen mit Entfernung der Cervix uteri wissenschaftlich nicht zu objektivieren, demgegenüber sind die Langzeitrisiken hinsichtlich der Entstehung eines Zervixstumpfkarzinoms nicht hinweg zu diskutieren.

Der Beweis des Benefits für die Patientin hinsichtlich der rein laparoskopisch durchgeführten Hysterektomie steht ebenfalls aus, zumal sich mehr und mehr der Konsens herauskristallisiert, dass eine vaginale Hysterektomie, im Gegensatz zur rein laparoskopisch durchgeführten Hysterektomie mit mehreren Inzisionen im Bereich der Bauchdecke, den wohl noch am wenigsten traumatisierenden Eingriff darstellt.

Daher sollte in der Gynäkologie der Ausbildung in vaginalen Operationsverfahren gesteigerte Bedeutung gegeben werden, speziell vor dem Hintergrund der Tatsache, dass, wie z.B. in Großbritannien ermittelt, aus vorwiegend ausbildungstechnischen Gründen nur mehr ca. 11 \% aller Hysterektomien bei benignen Indikationen in der Prämenopause vaginal durchgeführt werden.

\subsection{Transperitoneale laparoskopische Zügelplastiken}

\subsection{Abdominale Vaginaefixatio z.B. nach Mc-Call}

\subsection{Paravaginale Kolpopexie - zusätzliche Suspension der deszendierten vorderen apikalen Vaginalwand als zusätzliche Leistung zur einfachen Kolposuspension \\ 1.15 Hohe Douglasverödung}

Die hohe Douglasverödung stellt eine separate Operationstechnik in Modifikation nach Moscovic dar. Der normale Peritonealverschluss bei vaginaler oder abdominaler Hysterektomie sollte Teil der Hauptleistung sein. Bei sehr weiteren Douglasverhältnissen ist die Gefahr der erneuten Deszensus- und Bruchsackbildung so hoch, dass hier eine spezielle, sehr aufwendige Technik der Douglasverödung mit Resektion des überschüssigen Peritoneums und hohem zirkulärem Verschluss des Douglas erfolgen muss. Dies ist eine sehr aufwendige Leistung, die einen hohen Zeitaufwand bedarf. Sie sollte im Einzelfall zusätzlich begründet sein, macht zusätzlich auch eine Ureterdarstellung notwendig, da ansonsten die Gefahr der Ureterläsion gegeben ist.

\subsection{Vaginale sakrospinale Fixation nach Amreich-Richter}

Hierbei handelt es sich um eine der kompliziertesten vaginalen Operationstechniken in der Gynäkologie überhaupt. Sie kann in keiner Weise Nebenleistung bei 11.27 sein.

Im Rahmen der LAVH ist diese Leistung als spezieller Eingriff möglich.

Getrennte Operationstechniken sind schon deshalb angezeigt, da einerseits die Indikation (großer Bruchsack der hinteren Vaginalwand) nur beherrscht werden kann durch aufwendige vaginale Präparation und Fixationstechnik, andererseits dieser Eingriff vor allen Dingen bei großen Scheidenbruchsackbildungen im Zustand nach länger zurückliegender Hysterektomie mit Beckenboden- und Bindegewebsschwäche durchgeführt wird.

\subsection{Laparoskopische Lymphonodektomie}

\subsubsection{Pelvines Lymphknotensampling}

\subsubsection{Pelvine Lymphonodektomie}

\subsubsection{Paraaortale Lymphonodektomie}

\section{$2 \quad$ Hysteroskopie}

Der transzervikal-hysteroskopische Zugang zur Rekonstruktion des Uteruscavums ist zwischenzeitlich als etabliert anzusehen. Hier ist der Benefit für die Patientin offenkundig, da sowohl eine Laparotomie wie eine organinvasive Uterotomie umgangen werden kann. Allerdings sind die Komplikationsmöglichkeiten der operativen Hysteroskopie hinsichtlich des aufwendigen operativ-technischen Umfeldes und der Sicherheitsaspekte nicht zu unterschätzen.

Die Grenzen der Methode werden dann erreicht, wenn durch die intraabdominale Einschwemmung des Distensionsmediums bei zu ausgedehnten Geweberesektionen oder Operationszeiten über 50 min. eine kardiorespiratorische Gefährdung auftritt. 
Die Endometriumablation kann mittlerweile als „etablierte“ Indikation zur Behandlung therapieresistenter Menometrorrhagien bei Hochrisiko-Patientinnen angesehen werden.

\subsection{Myomresektion}

\subsection{Polypektomie}

\subsection{Probeexzision Endometrium bzw. Partielle Endometriumresektion}

\subsection{Septumdissektion}

\subsection{Synechiolyse}

\subsection{Transkavitäre Tubenkatheterisierung bzw. Falloposkopie}

\subsection{Salpingoskopie}

Mit dieser Methodik gelingt es, prognostisch signifikante Befunde zu klassifizieren und damit die Untersuchung zu einem wichtigen Zusatzkriterium für die Abwägung der Erfolgschancen tuben-rekonstruktiver Eingriffe zu machen. Methodisch bedingte Limitierung von Fimbrientrichter und Ampulle, während sich die isthmische und proximale Tube aufgrund ihrer geringen Lumenweite der Betrachtung durch das Salpingoskop entzieht.

Transvaginal-transuterine Katheterisierung zur Beurteilung der Tube zwischen isthmoampullärem Übergang und uterinem Tubenostium.

\section{Die Rolle der abdominalen Kolposuspension, insbesondere endoskopischer Verfahren (Laparoskopie und „Retziusskopie“) im Operationsspektrum der Stressinkontinenz}

Kolposuspensionsplastiken sind etablierte Operationsverfahren im Gesamtspektrum der weiblichen Inkontinenzchirurgie. Die Fülle der operativ-technischen Varianten und die unterschiedliche Nomenklatur stellen den nicht schwerpunktmäßig damit befassten Operateur vor große Verständnisschwierigkeiten hinsichtlich der Bewertung der verschiedenen Verfahren und der Indikationsabgrenzung. Noch komplexer wird die Problematik, wenn die Möglichkeit der Durchführung von Kolposuspensionsplastiken per Endoskopie in die Diskussion eingebracht wird.

Vor diesem Hintergrund soll im folgenden zum einen versucht werden, eine Charakteristik der abdominalen Kolposuspension unter Einbeziehung neuer endoskopischer Verfahren zu erarbeiten, zum anderen eigene Pilotergebnisse zur Bewertung neuer Techniken und Abrundung der Thematik darzustellen.

\section{- Operationstechnische Prinzipien}

Die abdominale Kolposuspension oder Zystourethropexie bewirkt durch die Suspension der paraurethralen Vaginalfaszie an bindegewebigen Strukturen des kleinen Beckens, durch Anhebung des Blasenhalses und durch die Annäherung an die Symphyse, eine Aufhebung der Hypermobilität des urethrovesikalen Überganges und eine Verbesserung der Drucktransmission auf die Urethra. Ein weiterer kontinenzfördernder Effekt resultiert aus der Wiederherstellung der funktionellen Urethralänge und Erhöhung des Urethraverschlussdruckes.
Indikation und Diagnostik

Die abdominale Kolposuspension ist aufgrund ihres Wirkprinzips eine ausschließliche Inkontinenz-Operation, die nicht zum Ausgleich eines anatomischen Defektes dienen kann. Hieraus ergibt sich eine klare Abgrenzung der Indikation auf mittelschwere und schwere Stressinkontinenzprobleme ohne Deszensus vaginae. Im Falle des gleichzeitigen Vorliegens eines Deszensus vaginae anterior muss ein anderes operatives Verfahren gewählt oder eine abdominale Kolposuspension mit einer rekonstruktiven Maßnahme des Beckenbodens kombiniert werden.

Bei niedrigem Urethraverschlussdruck ist die abdominale Kolposuspension allen anderen Operationsverfahren überlegen. Im Vergleich zu vaginalen Operationsverfahren kann durch die abdominale Kolposuspension eine signifikant bessere Verkleinerung des retrovesikalen Winkels erzielt werden.

Den guten Kontinenzraten stehen zwei Problembereiche gegenüber: Zum einen ergibt sich eine, meist passagere, MiktionsMorbidität, wie Pollakisurie, zum anderen ist die Rate des postoperativen Auftretens von Rectocelen begünstigt.

Die Wahl des operativen Verfahrens bei Vorliegen einer Harninkontinenz erfolgt nach Klinik, urodynamischen Daten und dem patho-anatomischen Befund der Blasenhalsregion.

Im Vordergrund steht die klinische Befunderhebung.

Mittels Perineal-Sonographie gelingt neben der Darstellung von Symphyse, Harnblase und Urethraverlauf die Visualisierung des Rektums, womit die Beckenbodenregion als Gesamtgefüge beurteilt werden kann. Nur in Ausnahmefällen einer Rezidiv-Harninkontinenz, bei der es zu ausgeprägten periurethralen Vernarbungen bereits gekommen ist, bietet das laterale Cystogramm die Möglichkeit einer übersichtlicheren Darstellung des Urethraverlaufes. Zusätzlich dazu liefert die Perineal-Sonographie Details der entsprechenden Narbenregionen, so dass in diesen Fällen beide Untersuchungsverfahren komplementär anzuwenden sind. Die wesentlichste Information des bildgebenden Verfahrens ist die Lage des Blasenhalses in Bezug zur Symphysen-Unterkante. Bei einem hochstehenden Blasenhals sollte der Zugangsweg von abdominal, also im günstigsten Falle ein Kolposuspensions-Verfahren zur Korrektur, gewählt werden. Bei tiefstehendem Blasenhals ist in Abhängigkeit der übrigen Kriterien ein abdominales Verfahren häufig wünschenswert, erweist sich aus technischen Gründen (low vaginal capacity) jedoch als nicht möglich. In diesen Fällen muss die Wahl auf ein vaginales Verfahren fallen, wobei berücksichtigt werden muss, dass bei RezidivStress-Inkontinenz eine alleinige Re-Kolporrhaphie keine eigentliche Inkontinenz-Operation darstellt. Ein ergänzendes Verfahren ist deshalb erforderlich, wobei sich Schlingenoperationen bzw. supportive Bandeinlagen als Modifikationen anbieten.

Die verschiedenen Untersuchungsabschnitte einer urodynamischen Untersuchung liefern mit ihren Messwerten eine wichtige Grundlage für die Auswahl des entsprechenden Operationsverfahrens. Dabei vermag die initial durchgeführte Zystometrie eine sensorische oder motorische Urge-Komponente $\mathrm{zu}$ erkennen. Mit der Aufzeichnung eines Urethra-Ruhedruckprofils kann ein flacher Kurvenverlauf von einem eher normalen bis steilem Kur- 
venverlauf unterschieden werden. Da eine Kolporrhaphie zu einer Abflachung der Ruhedruckkurve führt, ist zur Verbesserung einer entsprechenden vesiko-urethralen Drucktransmission bei flachem Urethra-Ruhedruckprofil ein abdominales Vorgehen im Sinne einer Kolposuspension zu bevorzugen. Bei Rezidiv-Stressinkontinenz und flachem Urethra-Ruhedruckprofil stellt sich das Problem der „hypotonen Urethra“. Es kann hier am ehesten von einer operativ bedingten Abflachung (im Zustand nach Kolporrhaphie) ausgegangen werden. Das Urethra-Stressdruckprofil bestätigt die Diagnose der Stress-Harninkontinenz und gibt gleichzeitig Hinweise für das Ausmaß einer insuffizienten vesiko-urethralen Drucktransmission. Die Uro-Flow-Messung gibt Hinweise, inwieweit eine Blasenentleerungsstörung besteht.

- Spektrum der technischen Varianten der abdominalen Kolposuspensionsplastiken

Eine Schematisierung der Modifikationen der abdominalen Kolposuspension (Marshall 1949) ergibt sich aus der Platzierung der Nähte im Bereich der Vaginalfaszie und der Fixationspunkte im Bindegewebebereich des kleinen Beckens.

Operativ-technische Unterschiede in Abhängigkeit vom jeweiligen Zugangsweg

\section{Endoskopische Modifikationen}

Im Rahmen der Ausweitung der „minimal access surgery“ stellt sich die Frage nach der endoskopischen Durchführung abdominaler Kolposuspensionsplastiken.

\subsection{Kolposuspension per laparoskopischem Zugang}

Beim laparoskopischen Zugang zur abdominalen Kolposuspension kann transperitoneal wie extraperitoneal vorgegangen werden. Wird mit dem Laparoskop transumbilikal eingegangen, kann entweder der Troikar bzw. das Laparoskop intraperitoneal platziert werden oder ein Spezial-Troikar mit Ballondilatationsvorrichtung präperitoneal nach prävesikal zum Cavum Retzii unter Umgehung des Intraperitonealraumes geführt werden, um so das Laparoskop nach Entfernen des Ballons in der präperitonealen Höhle platzieren zu können.

Hat der Operateur sich für den laparoskopisch transperitonealen Zugang entschieden, wie bei Fallkonstellation mit gleichzeitig anzugehender intraperitonealer Pathologie, wird nach Platzierung des Laparoskops und der Hilfseinstiche im suprasymphysären Bereich das Blasenperitoneum unmittelbar an der Überkreuzungsstelle von Symphysenbogen und Lig. umbilicale laterale infrasymphysär transversal eröffnet. Anschließend erfolgt die Präparation des Cavum Retzii, ebenfalls möglichst stumpf, mittels Aquadissektion oder endoskopischem Präpariertupfer.

Bei Benutzung eines Spezialtroikars (Einmaltroikar) mit Vorrichtung zur stumpfen Ballondilatation des Cavum Retzii muss entsprechend den Anweisungen des Herstellers vorgegangen werden. Es muss betont werden, dass dieses Vorgehen, in Abhängigkeit vom speziellen Equipment, besonders übungsbedürftig ist.

Ansonsten wird beim extraperitoneal-transperitonealen Zugang wie unter „Retziusskopie“ beschrieben vorgegangen.

\subsection{Kolposuspension per retziusskopischem Zugang}

Beim retziusskopischen Zugang, also beim Eingehen mit dem Trokar extraperitoneal, entweder transumbilikal oder suprasymphysär, kann entweder eine stumpfe Präparation mit dem Operationslaparoskop vorgenommen oder ebenfalls ein Spezialballontrokar zur stumpfen Dilatation des Cavums benutzt werden. Bei Verzicht auf einen Ballondilatationstrokar sollte der Trokar für die offene Laparoskopie benutzt werden. Die Präparation des Cavum Retzii erfolgt dann wie bei der offenen Laparoskopie in analogen Schritten, schichtweise. Durch Aufnaht von Peritoneum und Faszie am Trokar wird die Einstichpforte gasdicht verschlossen und mittels $\mathrm{CO}_{2}$-Insufflation ein „Pneumocavum“ angelegt. Unter Sicht kann die Entfaltung des Cavum Retzii beobachtet werden. Ohne weitere Manipulation lässt sich das Cavum Retzii zunächst durch den $\mathrm{CO}_{2}$-Gasdruck darstellen. Zur weiträumigeren Präparation von Paraurethralbereich und Symphysenhinterwand bzw. Obturatorius- und Vaginalfaszie wird ein Präpariertupfer über den Arbeitskanal des Endoskops eingeführt. Möglichst atraumatisch wird die Präparation durchgeführt und der retrosymphysäre Bereich sowie die Faszie der vorderen Vaginalwand sorgfältig von Fettgewebe befreit. Einzelne Gefäße werden koaguliert.

Unabhängig vom endoskopischen Zugangsweg wird die Kolpofixation durchgeführt.

\section{Zusammenfassende Wertung der endoskopischen \\ Operationstechniken \\ Indikationsstellung}

Gerade in einem Bereich wie der Inkontinenzchirurgie mit einem fast unüberschaubaren Spektrum operationstechnischer Varianten kommt der richtigen Indikationsstellung eine besondere Bedeutung zu. Die abdominalen Kolposuspensionsplastiken stellen etablierte Operationsverfahren dar, die mit zu den am meisten verbreiteten und am besten evaluierten Stressinkontinenzoperationsverfahren gehören. Die Indikationsstellung für die Kolposuspensionsplastiken prinzipiell bzw. den speziellen Vergleich untereinander, werden sehr variabel gehandhabt. Prinzipiell muss unterschieden werden, ob die Kolposuspension primär oder sekundär, d. h. bei einer primären oder Rezidivinkontinenz eingesetzt wird. Letztendlich sollte betont werden, dass die Indikationsstellung zur differenzierten Fragestellung, ob abdominal oder vaginal vorgegangen werden muss bzw. welche Technik bei dem jeweiligen Zugang gewählt wird, von klinischen und urodynamischen Kriterien strengstens abhängig gemacht werden muss (siehe Kapitel Indikationsstellung). Von daher ergeben sich nicht die geringsten Unterschiede in der Indikation im Vergleich zur konventionellen Kolposuspension. Gerade bei den technisch sehr aufwendigen und übungsbedürftigen endoskopischen Kolposuspensionsplastiken darf von dem Grundsatz nicht abgewichen werden, dass nicht der endoskopische Zugang, sondern die Heilungsaussicht der Patientin im Vordergrund der Indikationsstellung steht.

\section{$4 \quad$ Falloposkopie}

\subsection{Transhysteroskopische Falloposkopie}

\subsubsection{Transzervikale Folloposkopie}




\section{Kolposkopisch gesteuerte Eingriffe - Lasertherapie}

\subsection{Diagnostische Eingriffe}

\subsubsection{Portio-PE}

\subsubsection{Vulva-PE}

\subsubsection{Vulva-Exzision}

\subsubsection{CK-Kürettage}

Die zytologische Diagnostik hat bis vor kurzem in Deutschland das Vorgehen im Bereich des unteren Genitaltraktes prädominiert. Längst ist aber bekannt, dass klinische Entscheidungen, die alleine auf der Zytodiagnostik beruhen, sehr häufig zu Fehlentscheidungen wie Unter- und Übertherapie führen.

Die kolposkopische Diagnostik, kombiniert mit der kolposkopisch gesteuerten Biopsie, ist heute Grundlage für das Management von präkanzerösen Veränderungen im unteren Genitaltrakt. Nur eine sorgfältige, histologisch gesicherte Diagnostik ermöglicht ein für die Patientin sicheres exspektatives Vorgehen als Alternative zu sofortigen Konisationen bei Dysplasien. Kontrolluntersuchungen sind gerade bei leichtgradigen Dysplasien sinnvoll, weil es bei einem Großteil der Patientinnen mit leichtgradigen Dysplasien zu einer Spontanremission kommt. Konisationen können so vermieden werden. Dies ist von besonderer Bedeutung, da das Durchschnittsalter von Patientinnen mit Dysplasien bei 30 Jahren liegt. Betroffen sind häufig Frauen mit noch nicht abgeschlossener Familienplanung. Konisationen führen aber nachgewiesenermaßen zu einer erheblichen Zunahme der Frühgeburtlichkeit bei späteren Graviditäten.

Auch bei der Therapie von vulvären Läsionen ist die kolposkopisch gesteuerte Biopsie heute Grundlage für Therapieoptionen. Insbesondere pruriginöse Läsionen werden mitunter häufig bei mangelhafter Diagnostik in ihrer Bedeutung richtig erkannt (Morbus Paget, VIN).

\subsection{Therapeutische Eingriffe an der Portio}

\subsubsection{Laservaporisation}

\subsubsection{Laserkonisation}

6.2.3 Messerkonisation

1. kolposkopisch gesteuert

2. diagnostisch

\subsubsection{Kombinierte Messerkonisation/Laservaporisation}

\subsubsection{LEETZ (Large Loop of the Transformation Z.)}

Während die diagnostischen Eingriffe - kolposkopisch gesteuerte Biopsien - durch Diagnoseoptimierung ein minimalinvasives Management ermöglichen und einen Beitrag zur Vermeidung unnötiger Eingriffe leisten, wird durch innovative Operationstechnik der Eingriff selbst als minimalinvasive Operation möglich.
Die Laservaporisation ist an der Universitäts-Frauenklinik mittlerweile ein Standardverfahren geworden. Vorteile dieses Verfahrens im Vergleich zu herkömmlichen Konisationen liegen auf der Hand: Der Eingriff kann praktisch immer ambulant durchgeführt werden. Bei 95\% aller Patienten kann dies auch ohne Lokalanästhesie erfolgen. Die Komplikationsraten nach Laservaporisation sind extrem niedrig. Schwere postoperative Blutungen wurden in den letzten 10 Jahren nicht beobachtet. Leichte Blutungen kommen in einer Frequenz von unter 1\% vor und lassen sich durch erneute Laserkoagulation oder Tamponade problemlos beherrschen. Komplikationen bei nachfolgenden Schwangerschaften sind nicht bekannt. Die Heilungs- bzw. Erfolgsrate liegt bei über $95 \%$ und damit im Bereich von Konisationen. Der Indikationsbereich reicht von virugenen persistierenden Läsionen bis hin zur CIN III bei ektozervikaler Lokalisation. Relativ seltene Indikationen sind Kontaktblutungen bei Zervizitis. Voraussetzungen für die erfolgreiche und für die Patientin sichere Anwendung von Laservaporisationen sind

1. eine kombinierte kolposkopisch-zytologisch-histologisch präoperative Sicherung des Befundes. Große klinische Erfahrung des Pathologen, des Zytologen und insbesondere des Kolposkopikers sind Voraussetzung für die einwandfreie Diagnostik.

2. Läsionen müssen komplett einsehbar sein.

3. Sorgfältige Nachkontrollen sind erforderlich. Eine entsprechende Compliance der Patientinnen ist eine weitere Voraussetzung.

Obwohl mittlerweile der größte Teil aller Patientinnen mit CIN mittels einer ambulanten Laservaporisation behandelt wird, ist die Durchführung einer Messerkonisation bzw. Laserkonisation in vielen Fällen unumgänglich. Indikationen sind insbesondere kolposkopisch nicht überschaubare endozervikale Läsionen, unklare persistierende zytologische Abstrichergebnisse, ausgedehnte ektozervikale Läsionen mit fraglich invasiver Komponente. Die manuell durchgeführte Laserkonisation hat sich gegenüber der Messerkonisation dabei nicht durchsetzen können, sie ist technisch schwierig. Ein neues Laserkonisationsverfahren mittels eines Handstückes wird noch erprobt. In besonderen Situationen, wie ausgedehnte endozervikale Läsionen und ins Scheidengewölbe reichende dysplastische Veränderungen, ist manchmal auch eine kombinierte Messerkonisation/Laservaporisation sinnvoll. Während die Messerkonisation die sichere Diagnostik intrazervikal sichert, ermöglicht die Laservaporisation ein moderates chirurgisches Vorgehen bei der Entfernung der häufig in das Scheidengewölbe ziehenden Veränderungen.

\subsection{Eingriffe an der Vulva}

\subsubsection{Kondylome}

\subsubsection{VIN III - bowenoide Papulose}

\subsubsection{Morbus Paget}

\subsubsection{Pruritus vulvae (HPV-bedingte Läsionen)}

Die Einführung der Lasertherapie im Bereich der Vulva hat die Vulvatherapie und -chirurgie von Präkanzerosen revolutioniert. Ausgedehnte chirurgische Eingriffe sind heute nur noch selten erforderlich. 
Die häufigste Indikation für ambulante Lasertherapien sind Vulvakondylome.

Die zur Verfügung stehenden medikamentösen Verfahren sind nach wie vor, auch nach der Einführung von Imiquimot, häufig langwierig und schmerzhaft. Insbesondere, wenn es sich um ausgedehnte Läsionen handelt und frustrane medikamentöse Therapieversuche vorausgegangen sind, wird von einer Mehrheit der Patientinnen die Lasertherapie präferiert. Vulvakondylome werden heute an der Universitäts-Frauenklinik Tübingen zu $98 \%$ ambulant und in Lokalanästhesie abgetragen. Inwiefern neue innovative immunologische Verfahren Verbesserung bringen, auch hinsichtlich der Rezidivraten, bleibt abzuwarten. Der Einsatz von Interferonen, Leukozytenultrafiltraten und therapeutischen Impfungen erfolgt in kontrollierten Studien.

Bei sehr ausgedehnten Läsionen, insbesondere bei hochgradigen Dysplasien - früher als bowenoide Papulose bekannt - muss in weniger als 5\% auch heute noch der Eingriff in Narkose erfolgen.

Der Lasereingriff ersetzt aber in solchen Situationen die für die meist jungen Patienten persönlich katastrophale Vulvektomie und ermöglicht so ein organerhaltendes Vorgehen. Auch bei semimalignen Tumoren, wie dem Morbus Paget, kommt die Laservaporisation als Alternative zur radikalen Chirurgie in Betracht.

\subsection{Eingriffe an der Vagina}

\subsubsection{Kondylome}

\subsubsection{VAIN I-III}

\subsubsection{VAIN I-III nach Hysterektomie}

Häufig finden sich HPV-verursachte Läsionen wie Kondylome oder Dysplasien nicht an einer Stelle des unteren Genitaltraktes, sondern sind multilokulär ausgedehnt und befallen auch die Vagina. Die Therapie der Wahl bei vaginalen Läsionen ist heute die Laservaporisation. Zu empfehlende alternative Verfahren existieren in diesem Bereich nicht.

Gerade die vaginal häufig schlecht zu erreichenden Läsionen lassen sich mittels Lasertherapie konkurrenzlos gut ohne oder mit Lokalanästhesie entfernen.

Ein immer häufiger werdendes und zunehmend zu beobachtendes Phänomen sind Dysplasien am Scheidenabschluss nach Hysterektomie. Betroffen sind häufig Patientinnen im fortgeschrittenen Alter bei Zustand nach Konisation und/oder Hysterektomie wegen vor Jahrzehnten festgestellten Dysplasien.

Vor Einführung der Laservaporisation kam in solchen Fällen nur die Kolpektomie als sehr umfangreiches und die Funktion der Scheide häufig zerstörendes Verfahren in Betracht. Der damit verbundene wochenlange stationäre Aufenthalt kann heute in praktisch allen Fällen durch eine ambulante Laservaporisation ersetzt werden.

Auch wenn es aus unbekannter Ursache bei diesen Patienten über die Jahre zu Rezidiven kommt, so kann doch durch Einsatz der Lasertherapie die Situation gut kontrolliert und immer wieder entfernt werden (Tab.13).

\section{$7 \quad$ Axilloskopie}

\section{Sentinel Lymphnode-Verfahren über TRACER}

\section{Myomoperationen}

Uteruserhaltende Myomoperationen stellen ein komplettes Spektrum subtiler organerhaltender Operationsverfahren dar, die je nach anatomischem Sitz des Myoms, Größe der Myome bzw. Anzahl der Myome (solitäres Myom oder multiple Myome), ggf. auch mit verschiedensten anatomischen Positionen, unterschieden werden.

Dabei sind hinsichtlich der minimalinvasiven Technik entweder der laparoskopische Zugang, der hysteroskopische Zugang oder der kombiniert laparoskopisch und hysteroskopische Zugang zur kompletten Myomexstirpation notwendig.

Die gesamte uteruserhaltende Myomchirurgie unterliegt einem strukturellen Wandel durch die Einführung laparoskopischer bzw. hysteroskopischer Operationstechniken.

Durch die Perfektionierung der Präparationsinstrumente und die Möglichkeit der Morcellierung mittels elektronischer Morcellatoren ist die Größe des Myoms bei der laparoskopischen Myomenukleation kein Problem mehr. Vielmehr muss hier die Problematik gesehen werden, dass bei noch nicht erfülltem bzw. prospektivem Kinderwunsch die Rekonstruktion im Vordergrund steht. Gerade hier liegen die Grenzen der Methode. Bei zu tief intramural gelegenen Myomen ist oftmals eine suffiziente Rekonstruktion der Uterotomie nur schwer möglich. Hier können im Extremfall Uterusrupturen bei nachfolgenden Schwangerschaften auftreten.

Die hysteroskopische Resektion submuköser Myome stellt die „minimalst“ invasive uteruserhaltende Myomoperation dar, wobei ausgedehnte hysteroskopische Operationen einen hohen Ausbildungsstand und eine akribische Berücksichtigung der Sicherheitsaspekte erfordern.

Je nach Schwierigkeitsgrad sollten die folgenden Myomoperationen als eigenständige Eingriffe unabhängig voneinander abrechenbar sein.

\subsection{Subseröse bzw. gestielte Myome ohne Notwendigkeit der Uteruswandrekonstruktion als solitäre Myome}

9.2 Mehrere Myome (3-4 Myome)

\subsection{Multiple Myome}

\subsection{Solitäre oder multiple intramurale Myome mit der Notwendigkeit der Uteruswandrekonstruktion nach Enukleation}

\subsection{Intraligamentäre Myome}


Kolposkopisch gesteuerte Eingriffe

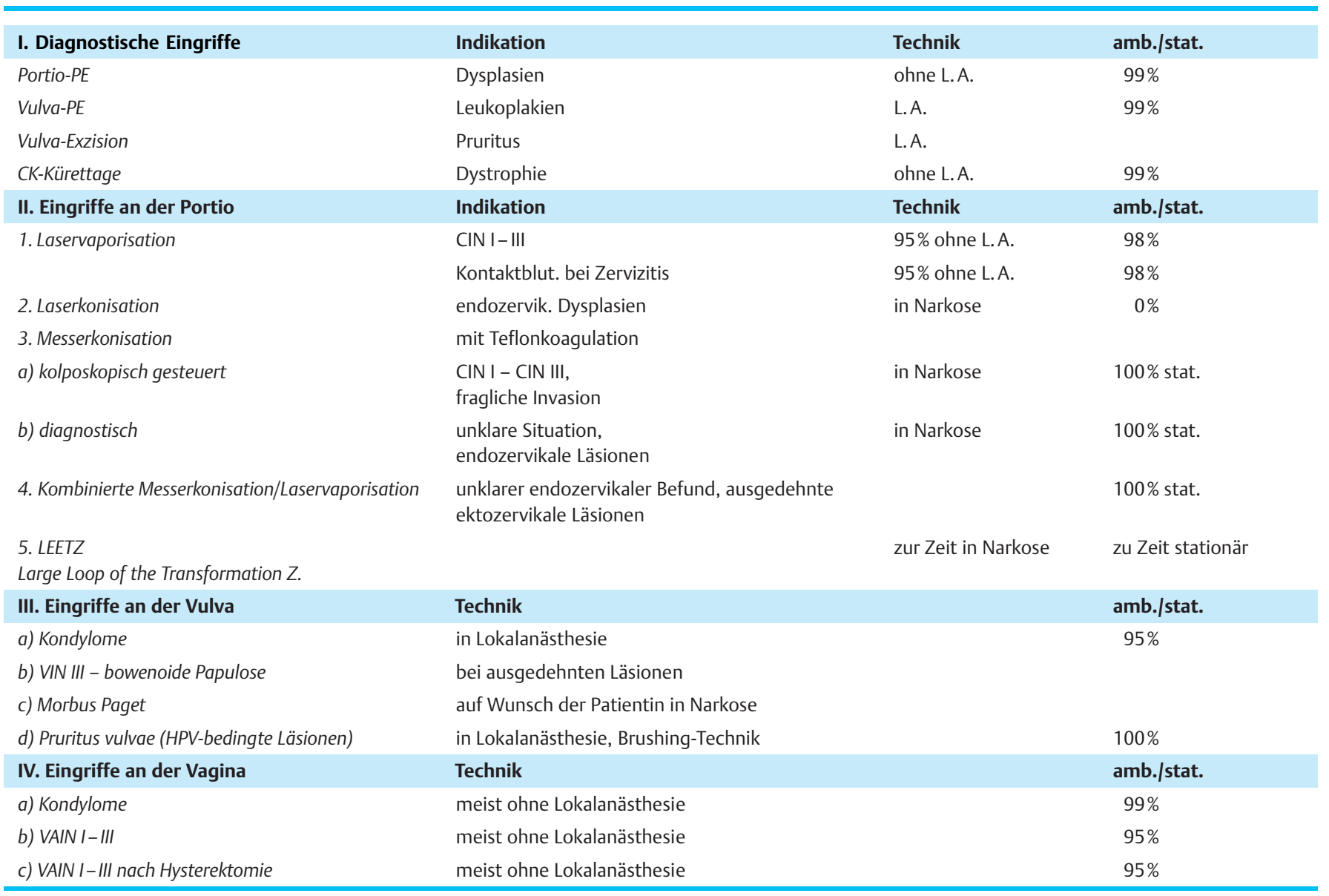

\subsection{Zervikale Myome, die nach intraperitoneal oder retroperitoneal entwickelt sind}

\subsection{Solitäre oder multiple Myome mit kompliziertem Situs}

wie z.B. in der Nähe der Uterinagefäßbündel oder des Tubenabgangs bei Patienten mit prospektivem Kinderwunsch oder Sterilitätsproblematik

\subsection{Sehr große intramurale Myome}

die die komplette Wandung des Uterus durchsetzen und einen erheblichen Uteruswanddefekt darstellen.

\subsection{Intrakavitäre submuköse gestielte Myome}

\subsection{Intrakavitäre submukös/Intramurale Myome mit unterschiedlichem Myomanteil intramural}

\subsection{Intravaginale Zervixmyome}

Aufgrund der unterschiedlichen operativen Vorgehensweisen und des unterschiedlichen anatomischen Sitzes sind die in den vorausgegangenen Ziffern benannten Myomoperationen jeweils separat abrechenbar.
- Myomexstirpation mit POR-8-Applikation

Die Verabreichung von Vasokonstriktiva ist nur bei starker Vaskularisierung notwendig und speziell bei individueller Reduktion des Blutverlustes indiziert. Die Resektionen von mehreren Myomen oder bei sehr großen oder bei kompliziertem Sitz der Myome sind teilweise nur dann möglich, wenn durch die Anwendung von vaso constructiva substanziell der Blutverlust bei der Enukleation der einzelnen Myome auf ein Minimum reduziert werden kann.

\section{Uteruswandrekonstruktion}

Im Falle tief intramural liegender Myome (in der Wandung des Uterus liegender Myome) ist bei prospektivem oder akutem Kinderwunsch die Rekonstruktion der Uteruswandung inkl. aller Wandschichtungen der schwierigste Eingriff bei der Myomenukleation. Hier sind aufwendige rekonstruktive Nahttechniken inkl. der Vereinigung aller durchtrennten Gewebeschichten des Uterus notwendig. Lediglich bei gestielten Myomen ist eine Uteruswandrekonstruktion nicht erforderlich. Hier sollte differenziert werden, und gerade bei der intramuralen Myomexstirpation ist die aufwendige Rekonstruktion schwieriger und subtiler als die Myomenukleation an sich. 


\section{Darmadhäsiolyse bei operativ-endoskopischen} Eingriffen

Hierbei handelt es sich um eine völlig selbständige Leistung, die in der Mehrzahl einen größeren Aufwand mit höherer Risikokonstellation hinsichtlich sekundärer Darmverletzungen, Peritonitis usw. mit sich bringt, als die Operation der EUG an sich.

90\% aller schwerwiegenden Komplikationen, die eine Re-Laparotomie, die Nachoperation einer Tubargravidität, ausmachen, sind Darmläsionen im Rahmen der Adhäsiolyse, um das Operationsgebiet hinsichtlich der Extrauteringravidität überhaupt zugänglich zu machen.

\section{Intraperitoneale Lavage}

Die intensive intraperitoneale Lavage als selbstständige Leistung ist nur dann notwendig, wenn durch akute, teils zurückliegende, teils frische Blutungen das gesamte kleine Becken so mit Blutkoageln ausgefüllt ist, dass als Ultima ratio zur Vermeidung einer Laparotomie zum Zugänglichmachen des Operationsgebietes und Vermeidung späterer Darmverwachsungen ein Herausspülen der Blutmassen, insbesondere der verhärteten Blutkoagel, notwendig ist. Im Falle der laparoskopischen Operation einer kleinen Tubargravidität ohne alte oder frische Blutkoagel ist sie ggf. nicht als separate Leistung notwendig.

\section{Videoüberwachung: Endoskopie über Videokette}

Die Videoüberwachung sollte eigentlich Videoskopie genannt werden, da das Operieren nicht mehr mit einem Auge von einem einzigen Mann ohne räumliches Sehen über ein Laparoskop oder ein Hysteroskop erfolgt, sondern durch videoskopische Übertragung auf einen großen Monitor erst kompliziertere laparoskopische wie hysteroskopische minimal invasive Operations- und Therapieverfahren möglich geworden sind. Bei einfachen Eingriffen, wie beispielsweise diagnostische Laparoskopie oder laparoskopische Sterilisationen ohne weitere intraperitoneale Pathologie oder Adhäsionen, ist im Gegensatz zu sonstigen videoskopischen Operationen kein räumliches Sehen über beide Augen und kein gleichzeitiges Operieren von einem ganzen Operationsteam unbedingt notwendig. Der Eingriff wird aber dadurch viel sicherer als beim Blick mit einem Auge durch das Endoskop selbst.

Von daher ist die Videoskopie originärer Bestandteil der modernsten innovativsten Entwicklung der minimalinvasiven Chirurgie. Ca. 90\% der endoskopischen Operationsverfahren sind so erst in den letzten Jahren zur Vermeidung eines Bauchschnittes mit allen gesundheitlichen und ökonomischen Konsequenzen möglich geworden, die verbleibenden 10\% der laparoskopischen Eingriffe, die nicht unbedingt auf Videoskopie angewiesen sind, werden aber durch die Videoüberwachung durch Videoskopie nicht nur wesentlich sicherer, sondern auch wesentlich präziser ermöglicht.

\section{- Zusammenfassende Wertung}

Da die meisten Operateure die entsprechende Fachkenntnis und das entsprechende technische Equipment nicht zur Verfügung haben, sollte bei immer komplexer werdenden minimal invasiven Operationsverfahren ganz speziell auf die jeweilige Indikation geachtet werden. Hiermit wird vermieden, dass eine zu häufige Vermischung von Haupt- und Nebenleistung resultiert und damit auch einer entsprechenden gezielten Falschabrechnung Vorbeuge geleistet wird. Gerade bei den hier angesprochenen Differenzierungen der Einzelleistungen muss in jedem Falle eine Indikation bestehen, da bei schematischer Abrechnung der verschiedenen Leistungen, wie z.B. Peritoneallavage, Ureterfreilegung, auch der Eindruck einer Kettenabrechnung entstehen könnte. Hier sollte im Sinne der gezielten Indikationsstellung und sachgerechten Abrechnung eine möglichst lückenlose Differenzierung erfolgen.

Ein spezielles Beispiel kann die Ureterolyse sein, da die laparoskopische Ureterolyse gerade bei Endometriose und Beckenwandfibrosierung für normale Operationszentren eine der schwierigsten und komplikationsreichsten Operationen überhaupt darstellt. Daher kann es nicht sein, dass dieser Eingriff immer oder häufig notwendig ist. Die Mehrzahl der Zentren ist hierzu gar nicht in der Lage. Die jeweilige Indikation hierzu muss gerechtfertigt sein, der Operateur muss über Ausbildung und technisches Equipment verfügen.

\section{- Thermische und athermische Operationstechniken}

Bisher wurden in der operativen Laparoskopie überwiegend elektrochirurgische Techniken, so die Hochfrequenztechnik und die Endokoagulationstechnik eingesetzt.

Um die präparativen Verfahren in der endoskopischen gynäkologischen Chirurgie zu modifizieren oder zu ergänzen, wurden vielfältige Versuche unternommen (Tab. 14).

\section{Tab. 14}

\section{Konventionelle präparative Verfahren}

\begin{tabular}{|c|c|}
\hline I. Thermisch & Unipolare HF-Elektrode \\
\hline a) Direkt-präparative Verfahren & $\begin{array}{l}\text { - } \text { unipolare } \\
\text { HF-Koagulation }\end{array}$ \\
\hline \multirow{2}{*}{$\begin{array}{l}\text { b) Indirekt-präparative Verfahren } \\
\text { (elektrische Hämostase, scharfe Präparation) }\end{array}$} & - Endokoagulation \\
\hline & - Bipolarkoagulation \\
\hline \multicolumn{2}{|l|}{ II. Nicht thermisch } \\
\hline \multicolumn{2}{|l|}{$\begin{array}{l}\text { Indirekt-präparative Verfahren: } \\
\text { scharfe Präparation nach: }\end{array}$} \\
\hline \multicolumn{2}{|l|}{ a) Endokoagulation } \\
\hline b) vasokonstriktiven Substanzen (Vasopression) & \\
\hline
\end{tabular}

\section{Die Nadelektrode}

Die monopolare und bipolare Präparationselektrode hat sich nicht nur für konventionelle sondern auch laparoskopische Techniken weitgehend durchgesetzt.

\section{- Die Elektrokoagulationstechnik}

Diese Technik ist ungefährlich für die Patientin, da der Stromfluss in einem separaten Stromkreis innerhalb des Instrumentes erfolgt. Die Gewebedestruktion ist jedoch erheblich, da der Punktkoagulator schon einen Durchmesser von $5 \mathrm{~mm}$ aufweist und dadurch per se schon eine Gewebeschädigung von $5 \mathrm{~mm}$ entsteht. Statt der Endokoagulationstechnik wird zur Verkleine- 
rung der Zone einer thermischen Gewebedestruktion zur Erzeugung einer lokalen Ischämie die endoskopische Vasopressin-Applikation eingesetzt. Die Gabe dieser Substanz erfordert aber eine anästhesiologische Überwachung, da kardiovaskuläre Risiken befürchtet werden können.

- Die $\mathrm{CO}_{2}$-Laser-Laparoskopie

Nachdem der $\mathrm{CO}_{2}$-Laser bereits für mehrere Indikationen, z. B. zur Adhäsiolyse am offenen Abdomen, in der gynäkologischen Reproduktionschirurgie eingeführt worden war, hatte erstmals Bruhat den $\mathrm{CO}_{2}$-Laser via Arbeitskanal laparoskopisch (SinglePuncture-Laparoskop) eingesetzt. 1981 führte Tadir ein SecondPuncture-Laparoskop zur laparoskpischen $\mathrm{CO}_{2}$-Laser-Präparation ein. Der $\mathrm{CO}_{2}$-Laser hat sich in den letzten Jahren zum Laser der 1. Wahl für die operative Laparoskopie entwickelt.

\section{GOÄ-Kommentierung}

Standard: Diagnostische Laparoskopie, Videoüberwachung (Tab. 15)

Tab. 15

\begin{tabular}{|lllc}
\hline Ziffer & Leistungstext & Punkte & EURO \\
\hline 0007 & vaginale Untersuchung & 0160 & 9,33 \\
\hline 0321 & $\begin{array}{l}\text { Sondierung/Katheterisierung } \\
\text { Gänge/Fistel }\end{array}$ & 0050 & 2,91 \\
\hline 1087 & Anlegen eines Portioadapters & 0055 & 3,21 \\
\hline 1096 & $\begin{array}{l}\text { Erweiterung des Gebärmutterhalses } \\
\text { durch Dehnung }\end{array}$ & 0148 & 8,63 \\
\hline $0700^{*}$ & Laparoskopie & 0800 & 46,63 \\
\hline 5295 & analog für Videoüberwachung, §6 & 0240 & 13,99 \\
\hline
\end{tabular}

* Ziffer 700 nie neben Ziffer 701

\section{Schwierigkeiten bei der Erstattung} Ziffer 5295 A GOÄ analog für Videoüberwachung

Die Berechnung der Ziffer 5295 A für die Videoüberwachung wird von einzelnen Krankenversicherungen bemängelt.

\section{Kommentar}

Die Videoüberwachung sollte eigentlich Videoskopie genannt werden, da das Operieren nicht mehr mit einem Auge von einer einzigen Person ohne die Möglichkeit des räumlichen Sehens über ein Laparoskop oder ein Hysteroskop erfolgt. Erst durch die videoskopische Übertragung auf einen großen Monitor sind komplizierte laparoskopische wie hysteroskopische minimalinvasive Operations- und Therapieverfahren möglich geworden. Bei einfachen Eingriffen, wie beispielsweise einer diagnostischen oder laparoskopischen Sterilisation ohne bestehende weitere intraperitoneale Pathologie oder Adhäsionen, ist im Gegensatz zu sonstigen videoskopischen Operationen kein räumliches Sehen über beide Augen und kein gleichzeitiges Operieren von einem ganzen Operationsteam unbedingt notwendig. Aber dadurch, dass mittels Videoskopie wie am offenen Abdomen operiert werden kann, wird ein noch so kleiner endoskopischer Eingriff viel sicherer als beim Betrachten mit einem Auge durch das Endo- skop. Von daher ist die Videoskopie ein dringender zusätzlicher Bestandteil der modernsten innovativen Entwicklung der minimalinvasiven Chirurgie.

Die Voraussetzung für die Abrechnung ist die entsprechende technische Ausstattung und die Erfahrung des Operateurs in der Interpretation der erhobenen Befunde.

Aus diesem Grund ist die Ziffer 5295 analog für die Videoüberwachung als selbständige Leistung neben der Ziffer 700 GOÄ berechnungsfähig.

\section{Endometrioseoperationen}

Leichtere Endometriosefälle profitieren von der endoskopischen Diagnostik, da sie so einer konsequenten Therapie zugeführt werden können. Die Endometriose nach dem AFS-Score I und II ist in den meisten Fällen laparoskopisch angehbar.

Sehr leicht wird auch, vergleichbar zur laparoskopischen Adhäsiolyse, die Grenze des endoskopisch Sinnvollen überschritten und eine komplexe Endometriose, z.B. mit Befall der Ovarien oder des Septum rectovaginale, endoskopisch nur insuffizient therapiert und damit die Rezidivrate noch erhöht. Häufig ist die Methode bei fortgeschrittener Endometriose überfordert. Darüber hinaus führt die Gefahr der Verletzung der Nachbarorgane, insbesondere des Ureters, zu einer inakzeptablen Komplikationsdichte.

\section{Elektrochirurgische Laser-Endometrioseresektionen bzw. Koagulationen}

Im Rahmen des $\S 6$ GOÄ sind selbständige ärztliche Leistungen, die in das Gebührenverzeichnis nicht aufgenommen sind, entsprechend einer nach Art, Kosten- und Zeitaufwand gleichwertigen Leistung des Gebührenverzeichnisses zu berechnen.

Um die Fertilität der Patientin erhalten zu können, ist eine möglichst radikale Entfernung der Endometriose erforderlich, und daher sind folgende Ziffern gemäß §6 analog in Ansatz zu bringen (Tab. 16).

\section{Tab. 16}

\begin{tabular}{|c|c|c|c|}
\hline Ziffer & Leistungstext & Punkte & EURO \\
\hline 0706 & $\begin{array}{l}\text { Analog: Elektrochirurgische Laser- } \\
\text { Endometrioseresektionen bzw. } \\
\text { Koagulationen entspricht Licht- oder } \\
\text { Laserkoagulation(en) zur Beseitigung } \\
\text { von Stenosen oder zur Blutstillung } \\
\text { bei endoskopischen Eingriffen, } \\
\text { je Sitzung, § } 6\end{array}$ & 0600 & 34,97 \\
\hline 2404 & $\begin{array}{l}\text { Analog: Endometrioseresektion im Be- } \\
\text { reich inneres Genital und Peritoneum } \\
\text { entspricht Exzision einer größeren } \\
\text { Geschwulst, § } 6\end{array}$ & 0554 & 32,29 \\
\hline
\end{tabular}


Operation bei Extrauteringravidität

- Operation bei Tubarabort

- Einfache Segmentresektion

- Segmentkoagulation

- Segmentresektion mit einzeitiger End/End-Anastomosierung

- Lineare Salpingotomie

- transampulläre Expression der Tubargravidität („milk-out“)

- Partielle Salpingektomie

- Totale Salpingektomie

- Operation bei Ovarialgravidität

Die mit der Tubargravidität assoziierte Sterilitätsproblematik rückt mehr und mehr in den Vordergrund. Der überwiegende Teil der Patientinnen kann aufgrund frühester Diagnostik der Extrauteringravidität einer geplanten und somit nicht notfallmäßigen Operation zugeführt werden. In etwa einem Drittel aller Fälle handelt es sich sogar um eine stehende Tubargravidität, bei der es nicht zur Tubenwandruptur oder zum Austritt von Blut in die freie Bauchhöhle gekommen ist (Tab. 17).

Tab. 17

\begin{tabular}{|c|c|c|c|}
\hline Ziffer & Leistungstext & Punkte & EURO \\
\hline 0007 & Vaginale Untersuchung & 0160 & 9,33 \\
\hline 0321 & $\begin{array}{l}\text { Sondierung/Katheterisierung } \\
\text { Gänge/Fistel }\end{array}$ & 0050 & 2,91 \\
\hline 0700 & Laparoskopie & 0800 & 46,63 \\
\hline 5295 & analog für Videoüberwachung & 0240 & 13,99 \\
\hline 1096 & $\begin{array}{l}\text { Erweiterung des Gebärmutterhalses } \\
\text { durch Dehnung }\end{array}$ & 0148 & 8,63 \\
\hline 1087 & Anlegen eines Portioadapters & 0055 & 3,21 \\
\hline 3172 & $\begin{array}{l}\text { Operative Darmmobilisation bei } \\
\text { Verwachsungen, als selbständige } \\
\text { Leistung (hier Adhäsiolyse der Becken- } \\
\text { wand, des Douglas-Raumes, des Uterus, } \\
\text { des Sigmas und des Netzes) - z. B. als } \\
\text { lleusprophylaxe }\end{array}$ & 1600 & 93,26 \\
\hline 1048 & Operation einer Extrauteringravidität & 2310 & 134,64 \\
\hline 3120 & $\begin{array}{l}\text { Diagnostische Peritonealspülung, als } \\
\text { selbständige Leistung } \\
\text { (hier: kontinuierliche Spülung zur } \\
\text { Ausräumung der Koagel) }\end{array}$ & 0300 & 17,49 \\
\hline
\end{tabular}

\section{Schwierigkeiten bei der Erstattung}

\section{Ziffer 3172 GOÄ - Darmadhäsiolyse}

Die Selbständigkeit der Leistung nach Ziffer 3172 GOÄ wird bemängelt. Die Krankenversicherungen behaupten, dass diese Leistung in der Hauptleistung (Operation einer Extrauteringravidität) als Zugangsleistung enthalten ist.

\section{Kommentar}

Die Darmadhäsiolyse bei operativ-endoskopischen Eingriffen kann eine selbständige Leistung darstellen, wenn sie nicht als Zugangsweg zur Extrauteringravidität dient, sondern z.B. als Ileusprophylaxe.
In diesem Fall ist die Darmadhäsiolyse zusätzlich neben der Ziffer 1048 GOÄ mit der Ziffer 3172 GOÄ berechnungsfähig und hat einen selbständigen Charakter neben dem Haupteingriff.

\section{Ziffer 3120 GOÄ - diagnostische Peritonealspïlung, als selbständige Leistung}

Die Selbständigkeit der Leistung nach Ziffer 3120 GOÄ wird bemängelt. Die Krankenversicherungen behaupten, dass diese Leistung in der Hauptleistung (Operation einer Extrauteringravidität) enthalten ist.

\section{Kommentar}

Die intensive intraperitoneale Lavage als selbständige Leistung ist nur dann notwendig und auch berechnungsfähig, wenn durch akute, teils zurückliegende, teils frische Blutungen das gesamte kleine Becken so mit Blutkoageln ausgefüllt ist, weil so eine ReLaparotomie vermieden werden kann, zum Zugänglichmachen des Operationsgebietes und zur Vermeidung späterer Darmverwachsungen, ein Herausspülen der Blutmassen, insbesondere der Blutkoageln notwendig ist. Im Falle der laparoskopischen Operation einer kleinen Tubargravidität ohne alte oder frische Blutkoagel ist diese Leistung nicht zusätzlich berechnungsfähig.

\section{Operationstyp Salpingotomie}

Hierunter ist die lineare Salpingotomie, das Aufschneiden der Tubenwand und das Herauspräparieren der Tubargravidität zu verstehen. Dies ist eines der aufwendigsten Verfahren, da zusätzlich zur Exstirpation der Tubargravidität gleichzeitig eine plastische Rekonstruktion der Tubenwand notwendig ist. Diese wiederum ist oft wesentlich schwieriger und muss hinsichtlich einer späteren Schwangerschaft subtiler durchgeführt werden als die Salpingotomie selbst.

Aus diesem Grund ist beim Operationstyp Salpingotomie zusätzlich zur Ziffer 1048 GOÄ (Operation einer Extrauteringravidität) die Ziffer 1145 (Operative Entfernung/Behandlung von Eierstock/Eileiter, einseitig) mit 1660 Punkten berechnungsfähig, weil dies dem Leistungsumfang entspricht.

\section{Operationstyp Segmentresektion mit einzeitiger End-zu-End-Anastomosierung}

Das einzeitige Vorgehen zur primären End-zu-End-Anastomosierung nach Segmentresektion ist den Fällen vorbehalten, bei denen die kontralaterale Tube fehlt oder einen schweren Tubenschaden aufweist. Da jedoch die Gewebsverhältnisse in den meisten Fällen für eine Anastomose sehr ungünstig sind, wird heute allgemein das zweizeitige Vorgehen befürwortet.

Für dieses aufwendige Verfahren der Operation einer Extrauteringravidität mit Erhalt der Fertilität muss zusätzlich zur Ziffer 1048 GOÄ (Operation einer Extrauteringravidität) die Ziffer 1148 bzw. 1149 GOÄ (Plastische Operation bei Tubensterilität einseitig bzw. beidseitig) mit 2500 bzw. 3500 Punkten in Ansatz gebracht werden.

\section{Operationen bei Infertilität}

- Salpingolyse- und Ovariolyse

- Fimbriolyse 
Tuboplastische Operationen bei distaler und proximaler Tubenpathologie bzw. Refertilisierungen

Obwohl die Rekonstruktion distaler Tubenpathologie als weit verbreitete Indikation zum laparoskopisch-rekonstruktiven Eingriff gilt, ist gerade diese Indikation sehr kritisch zu sehen. Hier sollte sich der Operateur bewusst sein, dass ein tubenrekonstruktiver Eingriff nur einmal vorgenommen werden kann, da eine nicht optimal durchgeführte Fimbriolyse, Fimbrioplastik oder Salpingotomie einen irreparablen Tubenschaden darstellt.

Hier muss kritisch unterschieden werden, ob der Eingriff nicht eher mikrochirurgisch erfolgen sollte bzw. eine Tubenrekonstruktion, beispielsweise bei dickwandiger oder gekammerter Sactosalpinx, eine unnötige Belastung für die Patientin darstellt und eine In-vitro-Fertilisation a priori bessere Erfolgsaussichten hätte (Tab. 18).

Tab. 18

\begin{tabular}{|c|c|c|c|}
\hline Ziffer & Leistungstext & Punkte & EURO \\
\hline 0007 & Vaginale Untersuchung & 0160 & 9,33 \\
\hline 0321 & $\begin{array}{l}\text { Sondierung/Katheterisierung } \\
\text { Gänge/Fistel }\end{array}$ & 0050 & 2,91 \\
\hline 1096 & $\begin{array}{l}\text { Erweiterung des Gebärmutterhalses } \\
\text { durch Dehnung }\end{array}$ & 0148 & 8,63 \\
\hline 1087 & Anlegen eines Portioadapters & 0055 & 3,21 \\
\hline 0700 & Laparoskopie & 0800 & 46,63 \\
\hline 5295 & analog für Videoüberwachung & 0240 & 13,99 \\
\hline 1112 & $\begin{array}{l}\times 2 \text {, Tubendurchblasung (Chromoper- } \\
\text { tubation) }\end{array}$ & $\begin{array}{l}0296 \\
\times 2\end{array}$ & $\begin{array}{l}17,25 \\
\times 2\end{array}$ \\
\hline 3172 & $\begin{array}{l}\text { Operative Darmmobilisation bei Ver- } \\
\text { wachsungen, als selbständige Leistung - } \\
\text { als selbständige Leistung, z. B. als } \\
\text { lleusprophylaxe }\end{array}$ & 1600 & 93,26 \\
\hline 1146 & $\begin{array}{l}\text { Operative Entfernung/Behandlung von } \\
\text { Eierstock/Eileiter, beidseitig (hier Sal- } \\
\text { pingo- und Ovarialyse bzw. Fimbriolyse) }\end{array}$ & 2220 & 129,40 \\
\hline 1829 & $\begin{array}{l}\times 2 \text {, Harnleiterfreilegung (Ureterolyse } \\
\text { bei retroperitonealer Fibrose und ggf. } \\
\text { intraperitonealen Verwachsungen des } \\
\text { Harnleiters. (hier bei endometriose- } \\
\text { oder verwachsungsbedingter retroperi- } \\
\text { tonealer Fibrose) }\end{array}$ & $\begin{array}{l}2590 \\
\times 2\end{array}$ & $\begin{array}{l}150,96 \\
\times 2\end{array}$ \\
\hline
\end{tabular}

\section{Schwierigkeiten bei der Erstattung \\ Ziffer 3172 GOÄ - Darmadhäsiolyse}

Die Selbständigkeit der Leistung nach Ziffer 3172 GOÄ wird bemängelt. Die Krankenversicherungen behaupten, dass diese Leistung in der Hauptleistung als Zugangsleistung enthalten ist.

\section{Kommentar}

Die Darmadhäsiolyse bei operativ-endoskopischen Eingriffen kann eine selbständige Leistung darstellen, wenn sie nicht als Zugangsweg zur Salpingolyse- und Ovariolyse oder Fimbriolyse dient, sondern z. B. als Ileusprophylaxe.
In diesem Fall ist die Darmadhäsiolyse zusätzlich neben der Ziffer 1048 GOÄ mit der Ziffer 3172 GOÄ berechnungsfähig und hat einen selbständigen Charakter neben dem Haupteingriff.

\section{Ziffer 1829 GOÄ - Ureterolyse bei retroperitonealer Fibrose}

Die Selbständigkeit der Leistung nach Ziffer 1829 GOÄ wird bemängelt. Die Krankenversicherungen behaupten, dass diese Leistung in der Hauptleistung enthalten ist.

\section{Kommentar}

Die Ziffer 1829 GOÄ ist nur dann als selbständige Leistung berechnungsfähig, wenn eine Freilegung der Beckenwand mit Ureterolyse bei starken Beckenwandverwachsungen, insbesondere bei endometriose- oder adhäsionsbedingter retroperitonealer Fibrose notwendig ist, da die endoskopische Freilegung der Beckenwände im Bereich der großen Gefäße zur Lösung des Ureters im Vergleich zum Eingriff an Tube, Ovar oder ganzer Adnexe in den meisten Fällen den schwierigeren Eingriff mit höherer Komplikationsrate darstellt.

- Fimbrioplastik

- Salpingostomie

- Salpingoneostomie

- Salpingotomie

- Salpingektomie

- Reanastomose (Tab. 19)

Tab. 19

\begin{tabular}{|c|c|c|c|}
\hline Ziffer & Leistungstext & Punkte & EURO \\
\hline 0007 & Vaginale Untersuchung & 0160 & 9,33 \\
\hline 0321 & $\begin{array}{l}\text { Sondierung/Katheterisierung } \\
\text { Gänge/Fistel }\end{array}$ & 0050 & 2,91 \\
\hline 1087 & Anlegen eines Portioadapters & 0055 & 3,21 \\
\hline 1096 & $\begin{array}{l}\text { Erweiterung des Gebärmutterhalses } \\
\text { durch Dehnung }\end{array}$ & 0148 & 8,63 \\
\hline 0700 & Laparoskopie & 0800 & 46,63 \\
\hline 5295 & analog für Videoüberwachung & 0240 & 13,99 \\
\hline 1112 & $\times 2$, Tubendurchblasung & $\begin{array}{l}0296 \\
\times 2\end{array}$ & $\begin{array}{l}17,25 \\
\times 2\end{array}$ \\
\hline 3172 & $\begin{array}{l}\text { Operative Darmmobilisation bei Ver- } \\
\text { wachsungen, als selbständige Leistung, } \\
\text { z. B. als lleusprophylaxe }\end{array}$ & 1600 & 93,26 \\
\hline 1149 & $\begin{array}{l}\text { Plastische Operation bei Tubensterilität, } \\
\text { beidseitig }\end{array}$ & 3500 & 204,01 \\
\hline 1829 & $\begin{array}{l}\times 2 \text { Harnleiterfreilegung (Ureterolyse bei } \\
\text { retroperitonealer Fibrose und ggf. intra- } \\
\text { peritonealen Verwachsungen des Harn- } \\
\text { leiters. (hier bei endometriose- oder ver- } \\
\text { wachsungsbedingter retroperitonealer } \\
\text { Fibrose). }\end{array}$ & $\begin{array}{l}2590 \\
\times 2\end{array}$ & $\begin{array}{l}150,96 \\
\times 2\end{array}$ \\
\hline
\end{tabular}

1. Die Ziffer 1148 bzw. 1149 GOÄ kann nicht für eine pertubare ovarielle Adhäsiolyse, sondern nur bei organrekonstruktiven Eingriffen an der/den Tube(n) in Ansatz gebracht werden.

2. Die Ziffer 1146 GOÄ (Operative Entfernung/Behandlung von Eierstock/Eileiter, beidseitig) für die Ovariolyse und Salpingolyse ist nicht neben der Ziffer 1149 GOÄ berechnungsfähig. 


\section{Schwierigkeiten bei der Erstattung} Ziffer 3172 GOÄ - Darmadhäsiolyse

Die Selbständigkeit der Leistung nach Ziffer 3172 GOÄ wird bemängelt. Die Krankenversicherungen behaupten, dass diese Leistung in der Hauptleistung als Zugangsleistung enthalten ist.

\section{Kommentar}

Die Darmadhäsiolyse bei operativ-endoskopischen Eingriffen kann eine selbständige Leistung darstellen, wenn sie nicht als Zugangsweg zur Fimbrioplastik, Salpingostomie, Salpingoneostomie, Salpingotomie, Salpingoektomie oder Reanastomose dient, sondern z. B. als Ileusprophylaxe.

In diesem Fall ist die Darmadhäsiolyse zusätzlich neben der Ziffer 1048 GOÄ mit der Ziffer 3172 GOÄ berechnungsfähig und hat einen selbständigen Charakter neben dem Haupteingriff.

\section{Ziffer 1829 GOÄ - Ureterolyse bei retroperitonealer Fibrose}

Die Selbständigkeit der Leistung nach Ziffer 1829 GOÄ wird bemängelt. Die Krankenversicherungen behaupten, dass diese Leistung in der Hauptleistung enthalten ist.

\section{Kommentar}

Die Ziffer 1829 GOÄ ist nur dann als selbständige Leistung berechnungsfähig, wenn eine Freilegung der Beckenwand mit Ureterolyse bei starken Beckenwandverwachsungen, insbesondere bei endometriose- oder adhäsionsbedingter retroperitonealer Fibrose notwendig ist, da die endoskopische Freilegung der Beckenwände im Bereich der großen Gefäße zur Lösung des Ureters im Vergleich zum Eingriff an Tube, Ovar oder ganzer Adnexe, in den meisten Fällen den schwierigeren Eingriff mit höherer Komplikationsrate darstellt.

\section{Operationen bei Adnextumoren}

- Zystektomie, Zystenexstirpation, Zystenfenestration, Zystenpunktion

- Dermoidektomie

- Endometrioseresektion

- Ovarektomie/Adnexektomie

- Ovarialtumorexstirpation (Zystexstirpation, Zystektomie, Dermoidektomie, Endometrioseexstirpation usw.)

- partielle Ovarektomie

- Elektrochirurgische bzw. Laserstichelungen multipler Zysten bei PCO-Syndrom

- transperitoneale Bergung über Bergesack

\section{Vorbemerkung}

Vor dem Hintergrund der Komplexität der Pathologie zystischer Adnextumoren kann keine 100\%ig sichere Selektion hinsichtlich des endoskopischen Zuganges bei vermutet benignen Ovarialtumoren erfolgen. Daher sollte im Zweifelsfalle der endoskopische Zugang, trotz optimaler Selektion, nur bei der Möglichkeit der intraoperativen Schnellschnittdiagnostik und des jederzeitigen Umschaltens auf eine Staging-Laparotomie gewählt werden. Die suffiziente Aufklärung der Patientin ist selbstverständlich. Auch sollte in diesen Fällen die laparoskopische Entfernung der Tumoren bzw. Adnexen möglichst im Bergesack erfolgen. Daher darf der endoskopische Zugang nur bei optimaler Selektion und nur bei der Möglichkeit der intraoperativen Gewebeschnittdiagnostik und des jederzeitigen Umschaltens auf eine Staging-Lapa- rotomie gewählt werden. Die suffiziente Aufklärung der Patientin ist selbstverständlich. Bei postmenopausalen Patientinnen ist die ablative Therapie die Therapie der Wahl (Tab. 20).

Tab. 20

\begin{tabular}{|c|c|c|c|}
\hline Ziffer & Leistungstext & Punkte & EURO \\
\hline 0007 & Vaginale Untersuchung & 0160 & 9,33 \\
\hline 0321 & $\begin{array}{l}\text { Sondierung/Katheterisierung } \\
\text { Gänge/Fistel }\end{array}$ & 0050 & 2,91 \\
\hline 1087 & Anlegen eines Portioadapters & 0055 & 3,21 \\
\hline 1096 & $\begin{array}{l}\text { Erweiterung des Gebärmutterhalses } \\
\text { durch Dehnung }\end{array}$ & 0148 & 8,63 \\
\hline 0700 & Laparoskopie & 0800 & 46,63 \\
\hline 0317 & Punktion eines Adnextumors & 0350 & 20,40 \\
\hline 3172 & $\begin{array}{l}\text { Operative Darmmobilisation bei Ver- } \\
\text { wachsungen, als selbständige Leistung } \\
\text { (hier Adhäsiolyse des Sigmas und des } \\
\text { Netzes) }\end{array}$ & 1600 & 93,26 \\
\hline 1829 & $\begin{array}{l}\text { Harnleiterfreilegung (Ureterolyse bei } \\
\text { retroperitonealer Fibrose und ggf. intra- } \\
\text { peritonealen Verwachsungen des Harn- } \\
\text { leiters (hier bei endometriose- oder ver- } \\
\text { wachsungsbedingter retroperitonealer } \\
\text { Fibrose). }\end{array}$ & 2590 & 150,96 \\
\hline 1145 & $\begin{array}{l}\text { Operative Entfernung/Behandlung von } \\
\text { Eierstock/Eileiter, einseitig }\end{array}$ & 1660 & 96,76 \\
\hline
\end{tabular}

\section{Anmerkung \\ Ovarialzystenstichelung bei PCO-Syndrom mittels Elektrode oder Laser}

Die zusätzlich Ovarialzystenstichelung bei PCO-Syndrom mittels Elektrode oder Laser ist separat mit der Ziffer 706 analog in Ansatz zu bringen.

\section{Schwierigkeiten bei der Erstattung Ziffer 3172 GOÄ - Darmadhäsiolyse}

Die Selbständigkeit der Leistung nach Ziffer 3172 GOÄ wird bemängelt. Die Krankenversicherungen behaupten, dass diese Leistung in der Hauptleistung als Zugangsleistung enthalten ist.

\section{Kommentar}

Die Darmadhäsiolyse bei operativ-endoskopischen Eingriffen kann eine selbständige Leistung darstellen, wenn sie nicht als Zugangsweg zur Operation von Adnextumoren dient, sondern z.B. als Ileusprophylaxe.

In diesem Fall ist die Darmadhäsiolyse zusätzlich neben der Ziffer 1048 GOÄ mit der Ziffer 3172 GOÄ berechnungsfähig und besitzt einen selbständigen Charakter neben dem Haupteingriff.

\section{Ziffer 1829 GOÄ - Ureterolyse bei retroperitonealer Fibrose}

Die Selbständigkeit der Leistung nach Ziffer 1829 GOÄ wird bemängelt. Die Krankenversicherungen behaupten, dass diese Leistung in der Hauptleistung enthalten ist. 
Kommentar

Die Ziffer 1829 GOÄ ist nur dann als selbständige Leistung berechnungsfähig, wenn eine Freilegung der Beckenwand mit Ureterolyse bei starken Beckenwandverwachsungen, insbesondere bei endometriose- oder adhäsionsbedingter retroperitonealer Fibrose notwendig ist, da die endoskopische Freilegung der Beckenwände im Bereich der großen Gefäße zur Lösung des Ureters im Vergleich zum Eingriff an Tube, Ovar oder ganzer Adnexe in den meisten Fällen den schwierigeren Eingriff mit höherer Komplikationsrate darstellt.

Die Ureterolyse stellt nur dann eine separate Leistung dar, wenn eine komplette Freilegung der Beckenwand und Ureterolyse bei starken Beckenwandverwachsungen, insbesondere bei Endometriose, Adhäsionen bzw. retroperitonealer Fibrosierung erforderlich ist; dann ist eine übermäßig komplizierte Operation notwendig, da die endoskopische Freilegung der Beckenwände im Bereich der großen Gefäße zur Lösung des Ureters im Vergleich zum Eingriff an Tube, Ovar oder ganzer Adnexe in den meisten Fällen den schwierigeren Eingriff mit höherer Komplikationsrate darstellt. In diesem Fall handelt es sich um einen separaten und eigenständig berechenbaren Eingriff. Falls die genannten Bedingungen nicht erfüllt werden, liegt auch keine separate Leistung vor.

\section{Operationen bei Myomen}

- Operation bei gestieltem Myom

- Operation bei subserösem Myom

- Operation bei intra- und transmuralem Myom

- Operation bei submukösem und intrakavitärem Myom

- Operation bei zervikalem Myom

- Operation bei intraligamentärem Myom
Tab. 21

\begin{tabular}{|c|c|c|c|}
\hline Ziffer & Leistungstext & Punkte & EURO \\
\hline 0007 & Vaginale Untersuchung & 0160 & 9,33 \\
\hline 0321 & $\begin{array}{l}\text { Sondierung/Katheterisierung } \\
\text { Gänge/Fistel }\end{array}$ & 0050 & 2,91 \\
\hline 1096 & $\begin{array}{l}\text { Erweiterung des Gebärmutterhalses } \\
\text { durch Dehnung }\end{array}$ & 0148 & 8,63 \\
\hline 1087 & Anlegen eines Portioadapters & 0055 & 3,21 \\
\hline 0700 & Laparoskopie & 0800 & 46,63 \\
\hline 1800 & $\begin{array}{l}\text { analog für manuelle elektronische Mor- } \\
\text { cellierung entspricht Zertrümmerung } \\
\text { und Entfernung von Blasensteinen unter } \\
\text { endoskopischer Kontrolle, je Sitzung, § } 6\end{array}$ & 1480 & 86,27 \\
\hline.$/ .700$ & abzüglich: diagnostische Laparoskopie & -800 & $-46,63$ \\
\hline 0264 & analog für vaso constriktion, $\S 6$ & 0120 & 7,00 \\
\hline 1162 & $\begin{array}{l}\text { Abdominale Myomenukleation - } \\
\text { subseröses Myom }\end{array}$ & 1850 & 107,83 \\
\hline 1162 & $\begin{array}{l}\text { Abdominale Myomenukleation - } \\
\text { intraligamentäres Myom }\end{array}$ & 1850 & 107,83 \\
\hline 1829 & $\begin{array}{l}\times 2 \text { Harnleiterfreilegung (Ureterolyse bei } \\
\text { retroperitonealer Fibrose und ggf. intra- } \\
\text { peritonealen Verwachsungen des Harn- } \\
\text { leiters. (hier bei druckbedingter retrope- } \\
\text { ritonealer Fibrose) - bei intraligamentä- } \\
\text { rem oder laterozervikalem Myomsitz - }\end{array}$ & $\begin{array}{l}2590 \\
\times 2\end{array}$ & $\begin{array}{l}150,96 \\
\times 2\end{array}$ \\
\hline 1160 & analog für Uterusrekonstruktion & 2770 & 161,46 \\
\hline
\end{tabular}

\section{Kommentar}

Die Ziffer 1829 GOÄ ist nur dann als selbständige Leistung berechnungsfähig, wenn eine Freilegung der Beckenwand mit Ureterolyse bei starken Beckenwandverwachsungen, insbesondere bei endometriose- oder adhäsionsbedingter retroperitonealer Fibrose, notwendig ist, da die endoskopische Freilegung der Beckenwände im Bereich der großen Gefäße zur Lösung des Ureters im Vergleich zum Eingriff an Tube, Ovar oder ganzer Adnexe in den meisten Fällen den schwierigeren Eingriff mit höherer Komplikationsrate darstellt. Diese Ziffer kann nur bei intraligamentärem oder laterozervikalem Sitz des Myoms berechnet werden.

Die Ureterolyse stellt nur dann eine separate Leistung dar, wenn eine komplette Freilegung der Beckenwand und Ureterolyse bei starken Beckenwandverwachsungen, insbesondere bei Endometriose, Adhäsionen bzw. retroperitonealer Fibrosierung, notwendig wird; dann ist eine übermäßig komplizierte Operation notwendig, da die endoskopische Freilegung der Beckenwände im Bereich der großen Gefäße zur Lösung des Ureters im Vergleich zum Eingriff an Tube, Ovar oder ganzer Adnexe in den meisten Fällen den schwierigeren mit höherer Komplikationsrate darstellt. In diesem Fall handelt es sich um einen separaten und eigenständig berechenbaren Eingriff. Falls die genannten Bedingungen nicht erfüllt werden, liegt auch keine separate Leistung vor.

\section{Ziffer 264 analog für Vasokonstriktion}

Die Krankenversicherungen lehnt die Erstattung ab, da es sich nicht um eine selbständige Leistung im Sinne des § 6 Analogbewertung handele. Sie behaupten, diese Leistung sei eine flankie- 
rende Maßnahme, die in der Zielleistung 1162 (abdominale Myomenukleation) enthalten ist.

\section{Kommentar}

Die Verabreichung von vaso constrictiva ist nur bei starker Vaskularisierung notwendig und speziell bei individueller Reduktion des Blutverlustes indiziert. Die Resektionen von mehreren Myomen oder bei sehr großen Myomen oder bei kompliziertem Sitz der Myome sind teilweise nur dann möglich, wenn durch die Anwendung von vaso construktiva substanziell der Blutverlust bei der Enukleation der einzelnen Myome auf ein Minimum reduziert werden kann.

Daher stellt die Verabreichung von vaso constrictiva eine selbständige ärztliche Leistung dar und ist mit der Ziffer 264 analog in Ansatz zu bringen.

\section{Ziffer 1160 analog für Uterusrekonstruktion}

Die Krankenversicherungen behaupten, dass diese zusätzliche aufwendige und schwierige Leistung in der Zielleistung abdominale Myomenukleation enthalten und nicht als selbständige Leistung im Sinne des $\S 6$ analog zu berechnen ist.

\section{Kommentar}

Im Falle tief intramural liegender Myome (in der Wandung des Uterus liegender Myome) ist bei prospektivem oder akutem Kinderwunsch die Rekonstruktion der Uteruswandung inklusive aller Wandschichten der schwierigste Eingriff bei der Myomenukleation. Hier sind aufwendige rekonstruktive Nahttechniken inklusive der Vereinigung aller durchtrennten Gewebeschichten des Uterus notwendig. Lediglich bei gestielten Myomen ist eine Uteruswandresektion nicht erforderlich. Hier sollte differenziert werden, und gerade bei der intramuralen Myomenukleation ist die aufwendige Rekonstruktion schwieriger und subtiler, als die Myomenukleation selbst.

Deshalb muss festgehalten werden, dass bei der intramuralen Myomenukleation die Uteruswandrekonstruktion eine zusätzliche selbständige Leistung ist und mit der Ziffer 1160 analog gemäß § 6 GOÄ in Ansatz zu bringen ist.

\section{Ziffer 1162 GOÄ - Myomenukleation}

Die Krankenversicherungen behaupten, dass die Ziffer 1162 nur einmal abzurechnen ist, obwohl der Leistungstext dieser Ziffer die Einzahl definiert.

\section{Kommentar}

Der Leistungstext der Ziffer 1162 in der aktuellen GOÄ (abdominale Myomenukleation) schreibt nur in der Einzahl.

Je nach Schwierigkeitsgrad sind die folgenden Myomoperationen als eigenständige Eingriffe jeweils mit der Ziffer 1162 GOÄ bzw. 1137 GOÄ unabhängig voneinander und unabhängig von der Anzahl bei gleichem Myomsitz abrechnungsfähig.

- subseröse bzw. gestielte Myome ohne Notwendigkeit der Uteruswandrekonstruktion als solitäre Myome

- solitäre oder multiple intramurale Myome mit der Notwendigkeit der zusätzlichen Uteruswandrekonstruktion nach Enukleation

- intraligamentäre Myome
- zervikale Myome, die nach intraperitoneal oder retroperitoneal entwickelt sind

- solitäre oder multiple Myome mit kompliziertem Sitz wie z.B. in der Nähe der Uterinagefäßbündel oder des Tubenabganges bei Patienten mit prospektivem Kinderwunsch oder Sterilitätsproblematik

- sehr große intramurale Myome, die die komplette Wandung des Uterus durchsetzen und einen erheblichen Uteruswanddefekt darstellen

- intrakavitäre submukös gestielte Myome

- intrakavitäre submukös/intramurale Myome mit unterschiedlichem Myomanteil intramural

- intravaginale Zervixmyome

Diese Abrechnungsweise erfordert eine äußerst diffizile Dokumentation im Operationsbericht.

\section{Kommentar zur Ziffer 1800 analog für die manuelle elektronische Morcellierung}

Die Grundlage für die Möglichkeit der Enukleation von Myomen auf endoskopischem Wege und deren anschließenden Bergung aus der Bauchhöhle ist nur mit der Durchführung einer Morcellierung möglich. Bei der endoskopischen Morcellierung ist eine Laparotomie nicht notwendig. Da in der Ziffer 1800 die Leistung nach Ziffer 700 enthalten ist, muss bei der endoskopischen manuellen elektronischen Morcellierung die Ziffer 700 in Abzug gebracht werden.

\section{Operationen zur Uterusentfernung}

- laparoskopisch assistierte vaginale Hysterektomie ohne Adnexe

- laparoskopisch assistierte vaginale Hysterektomie mit Adnexe (Tab. 22)

\section{Schwierigkeiten bei der Erstattung}

\section{Ziffer 3172 GOÄ - Darmadhäsiolyse}

Die Selbständigkeit der Leistung nach Ziffer 3172 GOÄ wird bemängelt. Die Krankenversicherungen behaupten, dass diese Leistung in der Hauptleistung als Zugangsleistung enthalten ist.

Kommentar

Die Darmadhäsiolyse bei operativ-endoskopischen Eingriffen kann eine selbständige Leistung darstellen, wenn sie nicht als Zugangsweg zur LAVH dient, sondern z. B. als Ileusprophylaxe.

Aus diesem Grund ist die Darmadhäsiolyse zusätzlich mit der Ziffer 3172 GOÄ berechnungsfähig und besitzt einen selbständigen Charakter neben dem Haupteingriff.

\section{Ziffer 1829 GOÄ - Ureterolyse bei retroperitonealer Fibrose}

Die Selbständigkeit der Leistung nach Ziffer 1829 GOÄ wird bemängelt. Die Krankenversicherungen behaupten, dass diese Leistung in der Hauptleistung enthalten ist.

\section{Kommentar}

Die Ziffer 1829 GOÄ ist nur dann als selbständige Leistung berechnungsfähig, wenn eine Freilegung der Beckenwand mit Ureterolyse bei starken Beckenwandverwachsungen, insbesondere bei endometriose- oder adhäsionsbedingter retroperitonealer 
Tab. 22

\begin{tabular}{|c|c|c|c|}
\hline Ziffer & Leistungstext & Punkte & EURO \\
\hline 0007 & Vaginale Untersuchung & 0160 & 9,33 \\
\hline 0321 & $\begin{array}{l}\text { Sondierung/Katheterisierung } \\
\text { Gänge/Fistel }\end{array}$ & 0050 & 2,91 \\
\hline 1096 & $\begin{array}{l}\text { Erweiterung des Gebärmutterhalses } \\
\text { durch Dehnung }\end{array}$ & 0148 & 8,63 \\
\hline 1087 & Anlegen eines Portioadapters & 0055 & 3,21 \\
\hline 0700 & Laparoskopie & 0800 & 46,63 \\
\hline 1146 & $\begin{array}{l}\text { Operative Entfernung/Behandlung } \\
\text { von Eierstock/Eileiter, beidseitig } \\
\text { (hier: Ovariolyse) }\end{array}$ & 2220 & 129,40 \\
\hline 3172 & $\begin{array}{l}\text { Operative Darmmobilisation bei Ver- } \\
\text { wachsungen, als selbständige Leistung } \\
\text { (hier Adhäsiolyse des Sigmas und des } \\
\text { Netzes) }\end{array}$ & 1600 & 93,26 \\
\hline 1829 & $\begin{array}{l}\times 2 \text {, Harnleiterfreilegung (Ureterolyse } \\
\text { bei retroperitonealer Fibrose und ggf. } \\
\text { intraperitonealen Verwachsungen des } \\
\text { Harnleiters. (hier bei druck- oder ver- } \\
\text { wachsungsbedingter retroperitonealer } \\
\text { Fibrose) }\end{array}$ & $\begin{array}{l}2590 \\
\times 2\end{array}$ & $\begin{array}{l}150,96 \\
\times 2\end{array}$ \\
\hline 1138 & $\begin{array}{l}\text { Vaginale/abdominale Totalexstirpation } \\
\text { des Uterus, ohne Adenexentfernung } \\
\text { oder }\end{array}$ & 2770 & 161,46 \\
\hline 1139 & $\begin{array}{l}\text { Vaginale/abdominale Totalexstirpation } \\
\text { des Uterus, mit Adnexentferung }\end{array}$ & 3330 & 194,10 \\
\hline
\end{tabular}

Fibrose notwendig ist, da die endoskopische Freilegung der Beckenwände im Bereich der großen Gefäße zur Lösung des Ureters im Vergleich zum Eingriff an Tube, Ovar oder ganzer Adnexe in den meisten Fällen den schwierigen Eingriff mit höherer Komplikationsrate darstellt.

Die Ureterolyse stellt nur dann eine separate Leistung dar, wenn eine komplette Freilegung der Beckenwand und Ureterolyse bei starken Beckenwandverwachsungen, insbesondere bei Endometriose, Adhäsionen bzw. retroperitonealer Fibrosierung, notwendig wird; dann ist eine übermäßig komplizierte Operation notwendig, da die endoskopische Freilegung der Beckenwände im Bereich der großen Gefäße zur Lösung des Ureters im Vergleich zum Eingriff an Tube, Ovar oder ganzer Adnexe, in den meisten Fällen den schwierigeren Eingriff und mit höherer Komplikationsrate darstellt. In diesem Fall handelt es sich um einen separaten und eigenständig berechenbaren Eingriff. Falls die genannten Bedingungen nicht erfüllt werden, liegt auch keine separate Leistung vor.

\section{Operationen bei Deszensus und Harninkontinenz}

- Kolposuspensionsplastik

- vordere und hintere Kolporrhaphie mit Beckenbodenplastik

- Douglas-Verödung

- vaginale Sacropexie

- Abdominale Vaginae-Fixatio z. B. nach McCall

- paravaginale Kolpopexie als zusätzliche Suspension der deszendierenden vorderen apikalen Vaginalwand als zusätzliche Leistung zur einfachen Kolposuspension
Diese Eingriffe werden im Abschnitt der offenen Verfahren behandelt.

\section{Operative Therapie gynäkologischer Malignome}

\section{Zervixcarcinom Vorbemerkung}

Die typische Operation des Zervixcarcinom ist die abdominale Radikaloperation nach Wertheim-Meigs. Die vaginale Radikaloperation nach Schauta-Amreich hat in den letzten Jahren eine Renaissance erfahren. In der GOÄ werden beide Verfahren gleich bewertet gegenübergestellt. Zum Zeitpunkt des Inkrafttretens der GOÄ wurde überwiegend nur das Stadium I b operiert. Höhere Tumorstadien wurden fast ausschließlich bestrahlt. Die pelvine Lymphonodektomie wird zusätzlich zur radikalen Hysterektomie im Rahmen des abdominalen Eingriffs durchgeführt, bei der vaginalen Radikaloperation unterbleibt die pelvine Lymphonodektomie oder wird in einem separaten Eingriff ggf. endoskopisch vorgenommen. Die paraaortale Lymphonodektomie wurde zum Zeitpunkt des Inkrafttretens der GOÄ beim Kollumkarzinom praktisch nicht durchgeführt, in Einzelfällen höchstens als Lymphknotensampling, um daraus weitere Therapiekonsequenzen abzuleiten (z. B. Bestrahlung) (Tab. 23).

\section{Tab. 23}

\begin{tabular}{|c|c|c|c|}
\hline Ziff. & Leistungstext & Punkte & EURO \\
\hline 1166 & $\begin{array}{l}\text { Radikaloperation des Zervixkrebses, } \\
\text { vaginal oder abdominal, mit Entfernung } \\
\text { der regionären Lymphknoten }\end{array}$ & 4620 & 269,29 \\
\hline 1146 & $\begin{array}{l}\text { Ovarektomie, Ovariotomie, Salpingekto- } \\
\text { mie, Salpingotomie, Salpingolyse und/ } \\
\text { oder Neostomie durch vaginale oder } \\
\text { abdominale Eröffnung der Bauchhöhle, } \\
\text { beidseitig }\end{array}$ & 2220 & 129,40 \\
\hline 1829 & $\begin{array}{l}\text { Harnleiterfreilegung (Ureterolyse bei } \\
\text { retroperitonealer Fibrose und ggf. intra- } \\
\text { peritonealen Verwachsungen des Harn- } \\
\text { leiters) (hier bei tumor- und druckbe- } \\
\text { dingter retroperitonealer Fibrose) }\end{array}$ & 2590 & 150,96 \\
\hline 2580 & $\begin{array}{l}\text { analog für die komplette Freilegung und } \\
\text { Schonung des Nervus obturatorius ent- } \\
\text { spricht Freilegung und Durchtrennung } \\
\text { oder Exhairese eines Nerven, § } 6\end{array}$ & 0554 & 32,29 \\
\hline 2802 & $\begin{array}{l}\text { Freilegung und/oder Unterbindung eines } \\
\text { Blutgefäßes in der Brust- oder Bauch- } \\
\text { höhle, als selbständige Leistung }\end{array}$ & 2220 & 129,40 \\
\hline 2032 & $\begin{array}{l}\text { Anlage einer proximal gelegenen Spül- } \\
\text { und/oder Saugdrainage } \\
\text { (hier Anlage einer Easy-flow-Drainage) }\end{array}$ & 0250 & 14,57 \\
\hline 2015 & $\begin{array}{l}\text { Anlegen einer oder mehrerer Redondrai- } \\
\text { nagen (hier subcutane Redondrainage) }\end{array}$ & 0060 & 3,50 \\
\hline 1795 & $\begin{array}{l}\text { Anlegung einer perkutanen Harnblasen- } \\
\text { fistel durch Punktion einschließlich } \\
\text { Kathetereinlegung }\end{array}$ & 0273 & 15,91 \\
\hline
\end{tabular}


Schwierigkeiten bei der Erstattung

Ziffern 2802, 2580 analog, 1829, 1809 GOÄ neben 1166 GOÄ

Die Selbständigkeit der Leistungen nach den Ziffern 2802, 2580 analog, 1829 und 1809 GOÄ neben der Leistung nach Ziffer 1166 GOÄ wird bemängelt. Die Krankenversicherungen behaupten, dass diese Leistungen in der Hauptleistung (Radikaloperation des Zervixkrebses, vaginal oder abdominal, mit Entfernung der regionären Lymphknoten) enthalten ist.

\section{Kommentar zur Ziffer 1829 GOÄ}

In seltenen Fällen ist beim Zervixcarcinom der Ureter ein- oder beidseitig schon kranial der Parametrien in Tumorgewebe eingebettet, wodurch eine Harnleiterfreilegung bei tumor- und druckbedingter Fibrose erforderlich wird, die nicht bereits in der Hauptleistung (radikalen Hysterektomie) enthalten ist. Diese operative Leistung geht nämlich davon aus, dass der Ureter aus dem Parametrium freipräpariert werden muss, das in der Regel nicht tumorinfiltriert ist.

\section{Kommentar zur Ziffer 2580 analog}

Bei einer ausgedehnten Tumorausbreitung in den Lymphknoten der Fossa obturatoria wird von einigen Operateuren der Eingriff abgebrochen und die Region nachbestrahlt. Andernfalls muss der N. obturatorius in seinem gesamten Verlauf in besonderer Tiefe vom Plexus pelvicus bis zum Foramen obturatum aus dem Tumor herausgelöst werden. In diesen Fällen stellt dieser Teil der Operation einen selbständigen Operationsschritt dar und ist mit der Ziffer 2580 analog in Ansatz zu bringen.

\section{Kommentar zur Ziffer 2802 GOÄ}

Bei einer ausgedehnten Tumorausbreitung zur Beckenwand hin wird von einigen Operateuren der Eingriff abgebrochen und die Region nachbestrahlt. Wird eine radikale Tumorektomie auch unter Entfernung von freien Tumorkonglomeraten im kleinen Becken durchgeführt, müssen ggf. Blutgefäße im Stromgebiet der A. und V. iliaca externa, interna oder communis bis zum Hauptstamm (Aorta) freipräpariert oder ligiert werden. In diesen Fällen stellt dieser Teil der Operation einen selbständigen Operationsschritt dar. Selbstverständlich kann diese Ziffer nicht für die Unterbindung der A. und V. uterina angesetzt werden, da diese Leistung integraler Bestandteil der radikalen Hysterektomie ist (Tab. 24).

\section{Schwierigkeiten bei der Erstattung Ziffer 1809 neben der Ziffer 1167}

Die Selbständigkeit dieser Leistung nach Ziffer 1809 GOÄ wird bemängelt. Die Krankenversicherungen behaupten, dass diese Leistung in der Hauptleistung (Radikaloperation des Zervixkrebses, vaginal oder abdominal, mit Entfernung der Lymphstromgebiete, auch paraaortal) enthalten ist.

\section{Kommentar zur Ziffer 1809 neben Ziffer 1167 GOÄ}

Die Ziffer 1167 GOÄ ist gegenüber der Ziffer 1166 GOÄ mit 280 Punkten höher bewertet. Die Ziffer sieht ausschließlich die abdominale Vorgehensweise vor. Diese Ziffer umfasst als Zielleistung die typische radikale Hysterektomie mit Ureterpräparation, Präparation der Parametrien mit den darin enthaltenen Lymphknoten und Lymphbahnen und die Resektion der Scheidenmanschette. Zusätzlich ist die Entfernung paraaortaler Lymphknoten Teil der Zielleistung. Dabei kann es sich jedoch nur um ein
Tab. 24

\begin{tabular}{|c|c|c|c|}
\hline Ziff. & Leistungstext & Punkte & EURO \\
\hline 1167 & $\begin{array}{l}\text { Radikaloperation des Zervixkrebses, } \\
\text { abdominal, mit Entfernung der Lymph- } \\
\text { stromgebiete, auch paraaortal }\end{array}$ & 4900 & 285,61 \\
\hline 1809 & $\begin{array}{l}\text { totale retroperitoneale Lymphaden- } \\
\text { ektomie }\end{array}$ & 4610 & 268,71 \\
\hline 1146 & $\begin{array}{l}\text { Ovarektomie, Ovariotomie, Salpingekto- } \\
\text { mie, Salpingotomie, Salpingolyse und/ } \\
\text { oder Neostomie durch vaginale oder } \\
\text { abdominale Eröffnung der Bauchhöhle, } \\
\text { beidseitig }\end{array}$ & 2220 & 129,40 \\
\hline 1829 & $\begin{array}{l}\text { Harnleiterfreilegung (Ureterolyse bei } \\
\text { retroperitonealer Fibrose und ggf. intra- } \\
\text { peritonealen Verwachsungen des Harn- } \\
\text { leiters) (hier bei tumor- und druckbe- } \\
\text { dingter retroperitonealer Fibrose) }\end{array}$ & 2590 & 150,96 \\
\hline 2580 & $\begin{array}{l}\text { analog für die komplette Freilegung und } \\
\text { Schonung des Nervus obturatorius ent- } \\
\text { spricht Freilegung und Durchtrennung } \\
\text { oder Exhairese eines Nerven, § } 6\end{array}$ & 0554 & 32,29 \\
\hline 2802 & $\begin{array}{l}\text { Freilegung und/oder Unterbindung eines } \\
\text { Blutgefäßes in der Brust- oder Bauch- } \\
\text { höhle, als selbständige Leistung }\end{array}$ & 2220 & 129,40 \\
\hline 2032 & $\begin{array}{l}\text { Anlage einer proximal gelegenen Spül- } \\
\text { und/oder Saugdrainage (hier Anlage } \\
\text { einer Easy-flow-Drainage) }\end{array}$ & 0250 & 14,57 \\
\hline 2015 & $\begin{array}{l}\text { Anlegen einer oder mehrerer Redondrai- } \\
\text { nagen (hier subcutane Redondrainage) }\end{array}$ & 0060 & 3,50 \\
\hline 1795 & $\begin{array}{l}\text { Anlegung einer perkutanen Harnblasen- } \\
\text { fistel durch Punktion einschließlich } \\
\text { Kathetereinlegung }\end{array}$ & 0273 & 15,91 \\
\hline
\end{tabular}

Lymphknotensampling handeln. Dies ergibt sich zwingend aus einem Vergleich mit der Ziffer 1809 GOÄ (totale retroperitoneale Lymphadenektomie), die mit 4610 Punkten bewertet ist. Wäre diese Leistung in der Ziffer 1167 enthalten, entfielen auf die radikale Hysterektomie mit Ureterpräparation etc. nur 290 Punkte.

Die Ziffer 1167 GOÄ kann also beim Zervixcarcinom dann eingesetzt werden, wenn neben der radikalen Hysterektomie lediglich ein zusätzliches paraaortales Lymphknotensampling erfolgt.

\section{Korpuscarcinom Vorbemerkung}

Für das Korpuscarcinom sieht der Verordnungsgeber in der GOÄ keine eigenständige Leistungsposition vor. Zum Zeitpunkt des Inkrafttretens der GOÄ war die Standardoperation beim Korpuscarcinom die abdominale Hysterektomie mit beidseitiger Adnexektomie. In den letzten Jahren haben jedoch Techniken der radikalen Hysterektomie beim Korpuscarcinom zunehmende Bedeutung erlangt. Daneben hat auch die pelvine und teilweise die paraaortale Lymphonodektomie Eingang in das Behandlungskonzept gefunden. Gemäß diesen Ausführungen kommen je nach tatsächlich durchgeführter Operation folgende Abrechnungsmodalitäten in Betracht (Tab. 25). 


\begin{tabular}{|c|c|c|c|}
\hline Ziff. & Leistungstext & Punkte & EURO \\
\hline 1138 & $\begin{array}{l}\text { Vaginale/abdominale Totalexstirpation des Uterus ohne Adnexentfernung } \\
\text { oder }\end{array}$ & 2770 & 161,46 \\
\hline 1139 & $\begin{array}{l}\text { Vaginale/abdominale Totalexstirpation des Uterus mit Adnexentferung } \\
\text { oder }\end{array}$ & 3330 & 194,10 \\
\hline \multirow[t]{2}{*}{$\begin{array}{l}1166 \\
{ }^{*} \mathbf{1}\end{array}$} & $\begin{array}{l}\text { analog Radikaloperation des Korpuscarcinom, vaginal oder abdominal, mit Entfernung der regionären Lymph- } \\
\text { knoten entspricht Radikaloperation des Zervixcarcinom, vaginal oder abdominal, mit Entfernung der regionären } \\
\text { Lymphknoten, § } 6\end{array}$ & 4620 & 269,29 \\
\hline & oder & & \\
\hline $\begin{array}{l}1167 \\
* 2\end{array}$ & $\begin{array}{l}\text { analog Radikaloperation des Korpuscarcinom, vaginal oder abdominal, mit Entfernung der regionären Lymph- } \\
\text { knoten entspricht Radikaloperation des Zervixcarcinom, vaginal oder abdominal, mit Entfernung der Lymph- } \\
\text { stromgebiete, auch paraaortal, § } 6\end{array}$ & 4900 & 285,61 \\
\hline $\begin{array}{l}1145 \\
{ }^{*} 1,{ }^{*} 2\end{array}$ & $\begin{array}{l}\text { Ovarektomie, Ovariotomie, Salpingektomie Salpingotomie, Salpingolyse und/oder Neostomie durch vaginale } \\
\text { oder } \\
\text { abdominale Eröffnung der Bauchhöhle, einseitig }\end{array}$ & 1660 & 96,76 \\
\hline $\begin{array}{l}1146 \\
{ }^{*} 1,{ }^{*} 2\end{array}$ & $\begin{array}{l}\text { Ovarektomie, Ovariotomie, Salpingektomie Salpingotomie, Salpingolyse und/oder Neostomie durch vaginale } \\
\text { oder } \\
\text { abdominale Eröffnung der Bauchhöhle, beidseitig }\end{array}$ & 2220 & 129,40 \\
\hline $\begin{array}{l}1809 \\
* 2\end{array}$ & totale retroperitoneale Lymphadenektomie & 4610 & 268,71 \\
\hline 2580 & $\begin{array}{l}\text { analog für die komplette Freilegung und Schonung des Nervus obturatorius entspricht Freilegung und Durch- } \\
\text { trennung oder Exhairese eines Nerven, } § 6\end{array}$ & 0554 & 32,29 \\
\hline 2802 & Freilegung und/oder Unterbindung eines Blutgefäßes in der Brust- oder Bauchhöhle, als selbständige Leistung & 2220 & 129,40 \\
\hline 2032 & Anlage einer proximal gelegenen Spül- und/oder Saugdrainage (hier Anlage einer Easy-flow-Drainage) & 0250 & 14,57 \\
\hline 2015 & Anlegen einer oder mehrerer Redondrainagen (hier subcutane Redondrainage) & 0060 & 3,50 \\
\hline 1795 & Anlegung einer perkutanen Harnblasenfistel durch Punktion einschließlich Kathetereinlegung & 0273 & 15,91 \\
\hline
\end{tabular}

\section{Schwierigkeiten bei der Erstattung}

Ziffer 1809 neben Ziffer 1167 GOÄ

Ziffer 2580 analog und Ziffer 2802 neben Ziffer 1166 bzw. 1167 GOÄ Die Selbständigkeit der Leistungen nach den Ziffern 2802, 1809 und 2580 analog neben der Leistung nach Ziffer 1166 bzw. 1167 GOÄ wird bemängelt. Die Krankenversicherungen behaupten, dass diese Leistungen in der Hauptleistung enthalten ist.

\section{Kommentar zur Ziffer 1166 analog GOÄ *1}

Erfolgt die Hysterektomie als radikale Hysterektomie mit pelviner Lymphonodektomie, so ist dies die entsprechende Abrechnungsziffer, falls nicht gleichzeitig ein paraaortales Lymphknotensampling vorgenommen wird (dann GOÄ 1167). Neben dieser Ziffer ist dann bei durchgeführter Adnexektomie die Ziffern 1145 oder 1146 GOÄ abrechenbar.

\section{Kommentar zur Ziffer 1167 analog GOÄ *2}

Erfolgt die Hysterektomie als radikale Hysterektomie mit pelviner Lymphonodektomie und paraaortalem Lymphknotensampling, ist dies die entsprechende Abrechnungsziffer. Neben dieser Ziffer ist dann die Adnxetomie nach Ziffer 1145 oder 1146 GOÄ abrechenbar.

Kommentar zur Ziffer 2580 analog GOÄ *1, *2

Bei einer ausgedehnten Tumorausbreitung in den Lymphknoten der Fossa obturatoria wird von einigen Operateuren der Eingriff abgebrochen und die Region nachbestrahlt. Andernfalls muss der N. obturatorius in seinem gesamten Verlauf in besonderer Tiefe vom Plexus pelvicus bis zum Foramen obturatum aus dem Tumor herausgelöst werden. In diesen Fällen stellt dieser Teil der Operation einen selbständigen Operationsschritt dar und ist mit der Ziffer 2580 analog in Ansatz zu bringen.

Kommentar zur Ziffer 2802 analog GOÄ *1, *2

Bei einer ausgedehnten Tumorausbreitung zur Beckenwand hin wird von einigen Operateuren der Eingriff abgebrochen und die Region nachbestrahlt. Wird eine radikale Tumorektomie auch unter Entfernung von freien Tumorkonglomeraten im kleinen Becken durchgeführt, müssen ggf. Blutgefäße im Stromgebiet der A. und V. iliaca externa, interna oder communis bis zum Hauptstamm (Aorta) freipräpariert oder ligiert werden. In diesen Fällen stellt dieser Teil der Operation einen selbständigen Operationsschritt dar. Selbstverständlich kann diese Ziffer nicht für die Unterbindung der A. und V. uterina angesetzt werden, da diese Leistung integraler Bestandteil der radikalen Hysterektomie ist.

\section{Kommentar zur Ziffer 1809 analog GOÄ *2}

Die Ziffer 1167 GOÄ ist gegenüber der Ziffer 1166 GOÄ mit 280 Punkten höher bewertet. Die Ziffer sieht ausschließlich die abdominale Vorgehensweise vor. Die Ziffer umfasst die Zielleistung als typische radikale Hysterektomie mit Ureterpräparation, Präparation der Parametrien mit den darin enthaltenen Lymphknoten und Lymphbahnen und die Resektion der Scheidenmanschette. Zusätzlich ist die Entfernung paraaortaler Lymphknoten 


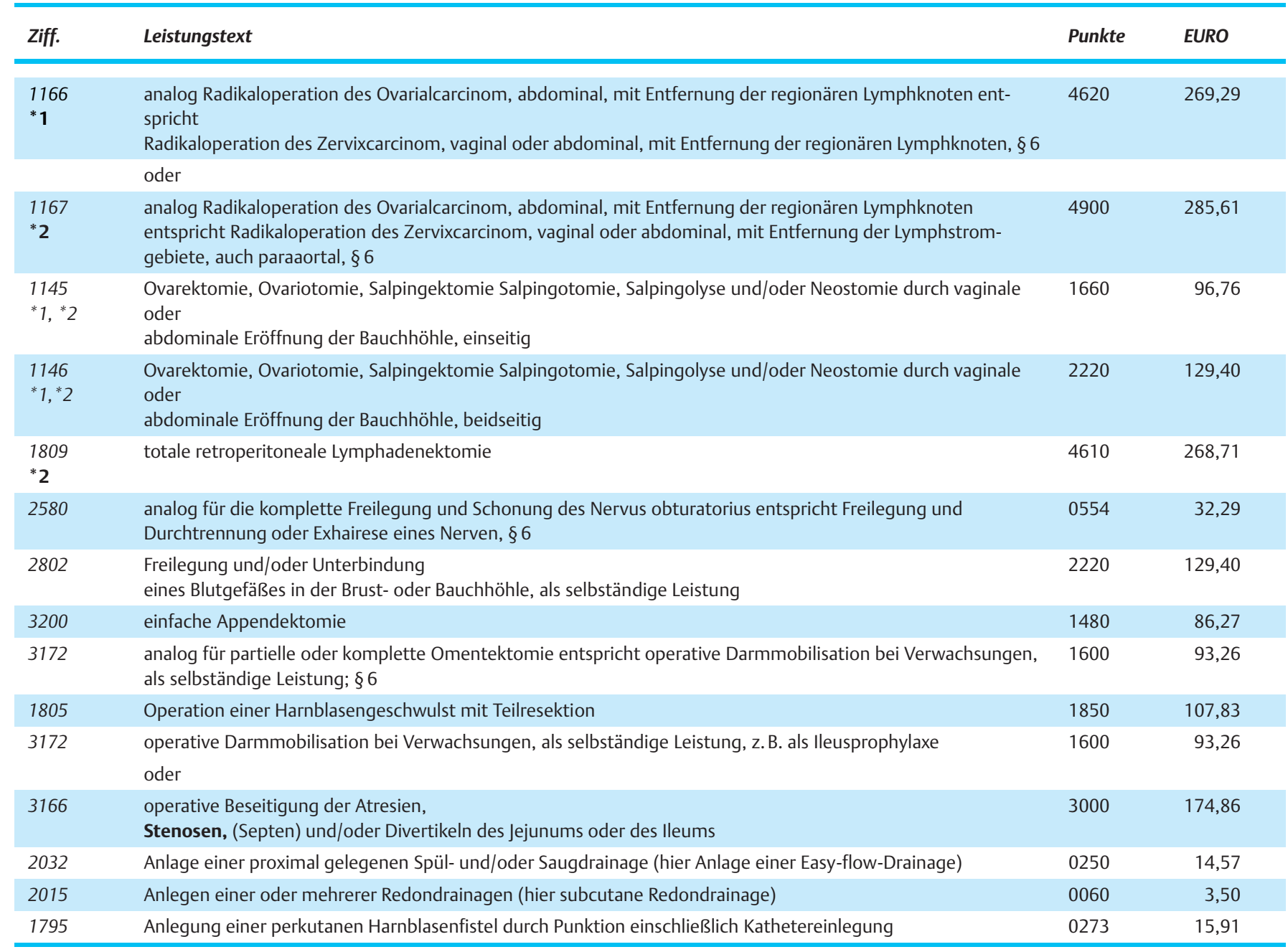

Teil der Zielleistung. Dabei kann es sich jedoch nur um ein Lymphknotensampling handeln. Dies ergibt sich zwingend aus einem Vergleich mit der Ziffer 1809 GOÄ (totale retroperitoneale Lymphadenektomie), die mit 4610 Punkten bewertet ist. Wäre diese Leistung in der Ziffer 1167 enthalten, entfielen auf die radikale Hysterektomie mit Ureterpräparation etc. nur 290 Punkte.

Die Ziffer 1167 GOÄ kann also beim Korpuscarcinom dann eingesetzt werden, wenn neben der radikalen Hysterektomie lediglich ein zusätzliches paraaortales Lymphknotensampling erfolgt.

\section{Ovarialcarcinom Vorbemerkung}

Auch für die operative Behandlung des Ovarialcarcinoms gibt es keine Ziffern, die dessen Therapie als Zielleistung enthalten. Die Abrechnung der operativen Maßnahmen muss daher ganz individuell an der tatsächlich erbrachten Leistung ausgerichtet werden. Gerade beim Ovarialcarcinom ist das Spektrum der zu erbringenden Leistungen sehr unterschiedlich: Im Stadium I + II kann in Einzelfällen bei noch bestehendem Kinderwunsch sogar organerhaltend operiert werden, während im Stadium III (ca. $60 \%$ aller Fälle bei der Primärdiagnose! ) mit diffuser Ausbreitung in der Peritonealhöhle eine möglichst maximale Tumorreduktion oder sogar Tumorfreiheit nur durch umfassende operative Teilschritte erreicht werden kann. Zum Zeitpunkt des Inkrafttretens der GOÄ wurde die operative Therapie in diesen Fällen häufig wegen sog. Inoperabilität abgebrochen, während heute in Zentren mit entsprechender Ausstattung und operativem Training in ca. $2 / 3$ der Fälle eine völlige Tumorfreiheit oder eine Tumorreduktion mit Resten unter $2 \mathrm{~cm}$ Durchmesser erreicht werden kann (Tab. 26).

\section{Schwierigkeiten bei der Erstattung Ziffer 1809 neben Ziffer 1167 GOÄ Ziffern 2580 analog, Ziffer 3172, Ziffer 1805, Ziffer 1809 und Ziffer 2802 neben Ziffer 1166 bzw. 1167 GOÄ}

Die Selbständigkeit der Leistungen nach den Ziffern 2802, 1809, 3172, 1805 und 2580 analog neben der Leistung nach Ziffer 1166 bzw. 1167 GOÄ wird bemängelt. Die Krankenversicherungen behaupten, dass diese Leistungen in der Hauptleistung enthalten sind. 
Kommentar zur Ziffer 1809 analog GOÄ *2

Die Ziffer 1167 GOÄ ist gegenüber der Ziffer 1166 GOÄ mit 280

Punkten höher bewertet. Die Ziffer sieht ausschließlich die abdominale Vorgehensweise vor. Diese Ziffer umfasst als Zielleistung die typische radikale Hysterektomie mit Ureterpräparation, Präparation der Parametrien mit den darin enthaltenen Lymphknoten und Lymphbahnen und die Resektion der Scheidenmanschette. Zusätzlich ist die Entfernung paraaortaler Lymphknoten Teil der Zielleistung. Dabei kann es sich jedoch nur um ein Lymphknotensampling handeln. Dies ergibt sich zwingend aus einem Vergleich mit der Ziffer 1809 GOÄ (totale retroperitoneale Lymphadenektomie), die mit 4610 Punkten bewertet ist. Wäre diese Leistung in der Ziffer 1167 enthalten, entfielen auf die radikale Hysterektomie mit Ureterpräparation etc. nur 290 Punkte.

Die Ziffer 1167 GOÄ kann also beim Ovarialcarcinom analog dann angesetzt werden, wenn neben der radikalen Hysterektomie lediglich ein zusätzliches paraaortales Lymphknotensampling erfolgt.

\section{Kommentar zur Ziffer 2580 analog}

Bei einer ausgedehnten Tumorausbreitung in den Lymphknoten der Fossa obturatoria wird von einigen Operateuren der Eingriff abgebrochen und die Region nachbestrahlt. Andernfalls muss der N. obturatorius in seinem gesamten Verlauf in besonderer Tiefe vom Plexus pelvicus bis zum Foramen obturatum aus dem Tumor herausgelöst werden. In diesen Fällen stellt dieser Teil der Operation einen selbständigen Operationsschritt dar und ist mit der Ziffer 2580 analog in Ansatz zu bringen.

\section{Kommentar zur Ziffer 2802 GOÄ}

Bei einer ausgedehnten Tumorausbreitung zur Beckenwand hin wird von einigen Operateuren der Eingriff abgebrochen und die Region nachbestrahlt. Wird eine radikale Tumorektomie auch unter Entfernung von freien Tumorkonglomeraten im kleinen Becken durchgeführt, müssen ggf. Blutgefäße im Stromgebiet der A. und V. iliaca externa, interna oder communis bis zum Hauptstamm (Aorta) freipräpariert oder ligiert werden. In diesen Fällen stellt dieser Teil der Operation einen selbständigen Operationsschritt dar. Selbstverständlich kann diese Ziffer nicht für die Unterbindung der A. und V. uterina angesetzt werden, da diese Leistung integraler Bestandteil der radikalen Hysterektomie ist.

\section{Kommentar zur Ziffer 3172 analog}

Die Netzresektion bis zum Querkolon bzw. bis zur Magenkurvatur muss heute Bestandteil der operativen Therapie des Ovarialcarcinoms sein. Für diese operative Maßnahme, die in dieser Form praktisch nur im Rahmen der Primärtherapie des Ovarialcarcinoms durchgeführt wird, gibt es in der GOÄ keine abgreifbare Ziffer. Andererseits ist dieser operative Schritt in keiner anderen operativen Leistung enthalten. Es ist daher zwingend geboten, die Netzresektion über die Analogziffer 3172 analog abzurechnen. Dieser Sachverhalt wird auch durch den Kommentar zur GOÄ für Ärzte von Dr. med. Brück, Dr. med. L. Krimmel, Dr. jur. R. Hess, Dr. med. B. Kleinken u. Dr. med. H.-J. Warlo bestätigt.
Kommentar zur Ziffer 1805 GÖ̈

Beim Ovarialcarcinom ist häufig bereits das Blasenperitoneum tumorinfiltriert, so dass eine vollständige Entfernung des Blasenperitoneum erforderlich wird. Diese Ausdehnung des Eingriffes stellt eine zusätzliche ärztliche Leistung dar, die nicht in der Hauptleistung enthalten und daher zusätzlich mit der Ziffer 1805 GOÄ in Ansatz zu bringen ist.

\section{Kommentar zur Ziffer 3172 oder 3166 GOÄ}

Beim Ovarialcarcinom ist das gesamte Darmkonvolut häufig tumorbedingt zu einem Konglomerat verwachsen, und es besteht eine Subileus-, Ileus- oder Stenosesymptomatik. In diesen Fällen muss eine systematische, häufig mehrere Stunden dauernde, Adhäsiolyse erfolgen, damit sodann entschieden werden kann, ob ggf. Darmsegmente zusätzlich reseziert werden müssen. Diese Leistung stellt einen eigenständigen Teilschritt der Operation dar und ist mit der Ziffer 3172 bzw. 3166 zusätzlich in Ansatz zu bringen. Sie ist nicht in anderen Leistungsziffern im Rahmen der operativen Therapie des Ovarialcarcinoms enthalten.

Die Ziffer kann nicht in Ansatz gebracht werden, wenn es sich lediglich um einzelne Verwachsungsstränge handelt, in die z.B. Uterus und Adnexe einbezogen sind. Die Adhäsiolyse ist hier Bestandteil der Zielleistung und kann ggf. über den Steigerungsfaktor berücksichtigt werden.

\section{Vulvacarcinom \\ Vorbemerkung}

Das Vulvacarcinom wird heute zunehmend individuell angepasst therapiert. Dies gilt einerseits für junge Patientinnen, bei denen funktionserhaltende Aspekte eine große Bedeutung haben, andererseits für hochbetagte Patientinnen, bei denen eingeschränkte Verfahren zur Verminderung der operativ bedingten Morbidität und Mortalität bedeutsam sind. Die GOÄ bietet differenzierte Abrechnungsmöglichkeiten gemäß den tatsächlich durchgeführten Maßnahmen (Tab. 27).

\section{Tab. 27}

\begin{tabular}{|llcc}
\hline Ziff. & Leistungstext & Punkte & EURO \\
\hline 1159 & $\begin{array}{l}\text { Abtragung großer Geschwulste } \\
\text { der äußeren Geschlechtsteile - } \\
\text { auch Vulvektomie - }\end{array}$ & 1660 & 96,76 \\
\hline $\begin{array}{l}\text { oder } \\
1165\end{array}$ & $\begin{array}{l}\text { Radikaloperation des Scheiden- und } \\
\text { Vulvakrebses }\end{array}$ & 3140 & 183,02 \\
\hline 1762 & $\begin{array}{l}\text { Inguinale Lymphknotenausräumung, } \\
\text { als selbständige Leistung }\end{array}$ & 1200 & 69,95 \\
\hline 1783 & $\begin{array}{l}\text { Pelvine Lymphknotenausräumung, } \\
\text { als selbständige Leistung }\end{array}$ & 1850 & 107,83 \\
\hline 1127 & $\begin{array}{l}\text { Vordere und hintere Scheidenplastik } \\
\text { mit Beckenbodenplastik } \\
\text { oder }\end{array}$ & 1660 & 96,76 \\
\hline 1126 & $\begin{array}{l}\text { Hintere Scheidenplastik mit } \\
\text { Beckenbodenplastik }\end{array}$ & 1290 & 75,19 \\
\hline 1125 & $\begin{array}{l}\text { oder } \\
\text { Vordere Scheidenplastik }\end{array}$ & & \\
\hline
\end{tabular}


Schwierigkeiten bei der Erstattung

Ziffern 1762 und 1783 neben Ziffer 1165 GOÄ

Die Selbständigkeit der Leistungen nach den Ziffern 1762 und 1783 neben der Leistung nach Ziffer 1166 bzw. 1167 GOÄ wird bemängelt. Die Krankenversicherungen behaupten, dass diese Leistungen in der Hauptleistung enthalten sind.

\section{Kommentar zur Ziffer 1159 GOÄ}

Bei eingeschränkten operativen Maßnahmen im Vulvabereich (z.B. Hemivulvektomie etc.) kann diese Ziffer in Ansatz gebracht werden.

\section{Kommentar zur Ziffer 1165 GOÄ}

Typische Leistungsziffer für die radikale Vulvektomie, bei der der Tumor weit im Gesunden unter Mitnahme der Labia minora und majora reseziert wird.

\section{Kommentar zur Ziffer 1762 GOÄ}

Die inguinale Lymphonodektomie ist eine eigenständige Leistung, die weder in der eingeschränkten noch in der radikalen Vulvektomie enthalten ist. Die inguinale Lymphonodektomie muss auch nicht zwingend im Rahmen der Vulvektomie vorgenommen werden, sondern stellt einen Eingriff mit differenzierter Indikationsstellung dar.

\section{Kommentar zur Ziffer 1783 GOÄ}

Bei Befall der pelvinen Lymphknoten kann es indiziert sein, über einen extraperitonealen Zugang die pelvinen Lymphknoten zusätzlich zu entfernen.

\section{Kommentar zur Ziffer 1127 oder 1126 oder 1125 GOÄ}

Bei der Vulvektomie wird der Scheideneingang gelegentlich stark verändert, so dass es in der Folge zu einem Scheidenprolaps kommen kann. Bei bereits bestehendem Prolaps des inneren Genitale kann es daher sinnvoll sein, während der primären Operation eine hintere Scheidenplastik und/oder Beckenbodenplastik durchzuführen. Diese Leistung stellt eine eigenständige Teilleistung dar, die nicht in der Leistung der Vulvektomie enthalten ist. Sie kann daher zweifelsfrei neben der entsprechenden Ziffer für die Vulvektomie abgerechnet werden. Entgegenstehende Ausführungen in einigen Kommentaren entbehren jeder medizinischen Grundlage. Sie beziehen sich lediglich auf die Nichtabrechenbarkeit dieser Leistung, wenn die Ziffer 1165 GOÄ für die Operation eines Scheidenkrebses mit Kolpektomie angesetzt wird.

\section{Vorbemerkung}

Die Aufstellung der GOÄ-Leistungsziffern stammt aus einer Zeit, seit der ein grundlegender Wandel in den Therapieverfahren stattgefunden hat. Daher treffen die Abrechnungs-Positionen in großen Teilen nicht mehr die heutigen standardmäßigen Operationsabläufe, die als zeitgemäß anzusehen sind. Dies gilt in besonders charakteristischer Weise für die heute üblichen Eingriffe an der Mamma. Während zum Zeitpunkt der Entwicklung der derzeit gültigen GOÄ bei Mammatumor-Operationen als Standard anzusehen war, dass zunächst eine Tumorexstirpation zur Malignitätssicherung vorgenommen wurde, die bei Tumornachweis zur modifiziert radikalen Mastektomie mit Ausräumung der ipsilateralen Axilla führte, wird nach den heutigen Maßstäben nach minimalinvasiver Diagnosesicherung durchweg die so genannte brusterhaltende Mammakarzinom-Therapie bevorzugt durchgeführt. Bei diesem Vorgehen ist das notwendige Therapiekonzept, das sowohl Heilungssicherheit wie ästhetische Ansprüche erfüllt, wesentlich schwieriger als die standardmäßige ablative Therapie früherer Zeiten.

\section{Segmentresektion}

- Segmentresektion ohne Pectoralismuskulatur

- Mastopexienähte (Tab. 28)

\section{Tab. 28}

\begin{tabular}{|llrr}
\hline Ziff. & Leistungstext & Punkte & EURO \\
\hline 2411 A & $\begin{array}{l}\text { Segmentresektion, entspricht Absetzen } \\
\text { einer Brustdrüse, §6 }\end{array}$ & 924 & 53,86 \\
\hline 2381 & $\begin{array}{l}\text { Einfache Hautlappenplastik } \\
\text { Anlegen einer oder mehrerer Redon- }\end{array}$ & 370 & 21,57 \\
\hline 0204 & Kompressionsverband & 80 & 8,04 \\
\hline 0206 & Tape-Verband & 70 & 5,54 \\
\hline
\end{tabular}

- Segmentresektion mit Pectoralismuskulatur

- Glanduläre Lappenplastik - lateral, medial, kranial und kaudal

- Mastopexienähte

- Brusterhaltende Therapie

- Axilladissektion Level I, II und III

- Sentinelverfahren

- Aufwendige Angleichung kontralaterale Mamma (Tab. 29) 
Tab. 29

\begin{tabular}{|c|c|c|c|}
\hline Ziff. & Leistungstext & Punkte & EURO \\
\hline \multicolumn{4}{|c|}{ Beispiel Mamma rechts: } \\
\hline $\begin{array}{l}2394 \\
4 \times\end{array}$ & $\begin{array}{l}\text { Implantation eines Rundstiellappens, einschließlich Modellierung am Ort (kranial, lateral, medial, kaudal) } \\
\text { - von innen - }\end{array}$ & 2200 & 128,23 \\
\hline 2415 & Rekonstruktion der Restbrust - aus den glandulären Rundstiellappen - & 2000 & 116,57 \\
\hline $2415 \mathrm{~A}$ & Remodellierung der Restbrust, entspricht Rekonstruktion der Restbrust, § 6 & 2000 & 116,57 \\
\hline $2417 \mathrm{~A}$ & $\begin{array}{l}\text { Rezentrierung der Mamille, entspricht operative Entnahme einer Mamille und intermistische Implantation } \\
\text { an anderer Körperstelle, § } 6\end{array}$ & 800 & 46,63 \\
\hline 2015 & Anlegen ein oder mehrerer Redondrainagen & 60 & 3,50 \\
\hline 5430 & Tumorszintigraphie einer Region & 1200 & 69,94 \\
\hline 2408 & Ausräumung des Lymphstromgebiets einer Axilla & 1100 & 64,12 \\
\hline 2583 & Neurolyse des Nervus thoracico dorsalis & 924 & 53,86 \\
\hline 2583 & Neurolyse des Nervus thoracicus longus & 924 & 53,86 \\
\hline 2583 & Neurolyse des Nervus intercostobrachialis & 924 & 53,86 \\
\hline 2015 & Anlegen einer oder mehrerer Redondrainagen & 60 & 3,50 \\
\hline 0204 & Kompressionsverband & 95 & 3,79 \\
\hline \multicolumn{4}{|c|}{ Kontralaterale Mamma: } \\
\hline 2414 & Reduktionsplastik der Mama & 2800 & 163,20 \\
\hline $2415 A$ & Remodellierung der Restbrust, entspricht Rekonstruktion der Restbrust, § 6 & 2000 & 116,57 \\
\hline $2417 \mathrm{~A}$ & $\begin{array}{l}\text { Rezentrierung der Mamille, entspricht operative Entnahme einer Mamille und Implantation an anderer } \\
\text { Körperstelle, § } 6\end{array}$ & 800 & 46,63 \\
\hline 2015 & Anlegen einer oder mehrerer Redondrainagen & 60 & 3,50 \\
\hline 0204 & Kompressionsverband & 95 & 3,79 \\
\hline
\end{tabular}


Tab. $\mathbf{3 0}$

\begin{tabular}{|c|c|c|c|}
\hline Ziff. & Leistungstext & Punkte & EURO \\
\hline $2412 A$ & $\begin{array}{l}\text { Segmentresektion mit Pectoralismusku- } \\
\text { latur, entspricht Absetzen einer Brust- } \\
\text { drüse, einschließlich Brustmuskulatur }\end{array}$ & 1400 & 81,60 \\
\hline $\begin{array}{l}2394 \\
4 \times\end{array}$ & $\begin{array}{l}\text { Implantation eines Rundstiellappens, } \\
\text { einschließlich Modellierung am Ort } \\
\text { (kranial, lateral, medial, kaudal) } \\
\text { - von innen - }\end{array}$ & 2200 & 128,23 \\
\hline $\begin{array}{l}2382 \\
4 \times\end{array}$ & $\begin{array}{l}\text { Schwierige Hautlappenplastik oder } \\
\text { Spalthauttransplantation (kranial, } \\
\text { lateral, medial, kaudal) - von außen - }\end{array}$ & 739 & 43,07 \\
\hline 2415 & $\begin{array}{l}\text { Rekonstruktion der Restbrust } \\
\text { - aus den glandulären Rundstiellappen - }\end{array}$ & 2000 & 116,57 \\
\hline $2415 A$ & $\begin{array}{l}\text { Remodellierung der Restbrust, entspricht } \\
\text { Rekonstruktion der Restbrust, § } 6\end{array}$ & 2000 & 116,57 \\
\hline $2417 A$ & $\begin{array}{l}\text { Rezentrierung der Mamille, entspricht } \\
\text { operative Entnahme einer Mamille und } \\
\text { Implantation an anderer Körperstelle, § } 6\end{array}$ & 800 & 46,63 \\
\hline 2015 & $\begin{array}{l}\text { Anlegen einer oder mehrerer } \\
\text { Redondrainagen }\end{array}$ & 60 & 3,50 \\
\hline 0204 & Kompressionsverband & 95 & 3,79 \\
\hline 0206 & Tape-Verband & 70 & 4,08 \\
\hline
\end{tabular}

Reduktionsmastektomie bei Makromastie Angleichung kontralaterale Seite (Tab. 31)

Tab. $\mathbf{3 1}$

\begin{tabular}{|c|c|c|c|}
\hline Ziffer & Leistungstext & Punkte & EURO \\
\hline \multicolumn{4}{|c|}{ Beispiel: Mamma rechts } \\
\hline 2414 & Reduktionsplastik der Mamma & 2800 & 163,20 \\
\hline $2415 \mathrm{~A}$ & $\begin{array}{l}\text { Reformierung der Restbrust aus den } \\
\text { glandulären Rundstiellappen, entspricht } \\
\text { Aufbauplastik der Mamma, einschließ- } \\
\text { lich Verschiebeplastik, §6 }\end{array}$ & 2000 & 116,57 \\
\hline $2415 A$ & $\begin{array}{l}\text { Remodellierung der Restbrust, ent- } \\
\text { spricht Rekonstruktion der Restbrust, } \S 6\end{array}$ & 2000 & 116,57 \\
\hline $2414 \mathrm{~A}$ & $\begin{array}{l}\text { Lifting des Hautmantels, entspricht } \\
\text { Reduktionsplastik Mamma, § } 6\end{array}$ & 2800 & 163,20 \\
\hline $\begin{array}{l}2382 \\
\times 4\end{array}$ & $\begin{array}{l}\text { Schwierige Hautlappenplastik (kranial, } \\
\text { lateral, medial, kaudal) - von außen - }\end{array}$ & 739 & 43,07 \\
\hline $\begin{array}{l}2394 \\
\times 4\end{array}$ & $\begin{array}{l}\text { Implantation eines Rundstiellappens, } \\
\text { einschließlich Modellierung am Ort } \\
\text { kranial, lateral, medial, kaudal) } \\
\text { - von innen - }\end{array}$ & 2200 & 128,23 \\
\hline $2417 A$ & $\begin{array}{l}\text { Rezentrierung der Mamille, entspricht } \\
\text { operative Entnahme einer Mamille und } \\
\text { intermistische Implantation an anderer } \\
\text { Körperstelle, § } 6\end{array}$ & 800 & 46,63 \\
\hline 2015 & $\begin{array}{l}\text { Anlegen einer oder mehrerer Redon- } \\
\text { drainagen }\end{array}$ & 60 & 3,50 \\
\hline 0204 & Kompressionsverband & 95 & 3,79 \\
\hline 0206 & Tape-Verband & 70 & 4,08 \\
\hline \multicolumn{4}{|c|}{ Kontralaterale Mamma: } \\
\hline 2414 & Reduktionsplastik der Mama & 2800 & 163,20 \\
\hline $2415 A$ & $\begin{array}{l}\text { Remodellierung der Restbrust, ent- } \\
\text { spricht Rekonstruktion der Restbrust, } \S 6\end{array}$ & 2000 & 116,57 \\
\hline $2414 A$ & $\begin{array}{l}\text { Lifting des Hautmantels, entspricht } \\
\text { Reduktionsplastik Mamma, Par. } 6\end{array}$ & 2800 & 163,20 \\
\hline $\begin{array}{l}2382 \\
4 \times\end{array}$ & $\begin{array}{l}\text { Schwierige Hautlappenplastik oder } \\
\text { Spalthauttransplantation (kranial, } \\
\text { lateral, medial, kaudal) }\end{array}$ & 739 & 43,07 \\
\hline $\begin{array}{l}2394 \\
4 \times\end{array}$ & $\begin{array}{l}\text { Implantation eines Rundstiellappens, } \\
\text { einschließlich Modellierung am Ort } \\
\text { (kranial, lateral, medial, kaudal) }\end{array}$ & 2200 & 128,23 \\
\hline $\begin{array}{l}2381 \\
4 \times\end{array}$ & $\begin{array}{l}\text { Einfache Hautlappenplastik (kranial, } \\
\text { lateral, medial, kaudal) }\end{array}$ & 370 & 18,07 \\
\hline $2417 \mathrm{~A}$ & $\begin{array}{l}\text { Rezentrierung der Mamille, entspricht } \\
\text { operative Entnahme einer Mamille und } \\
\text { intermistische Implantation an anderer } \\
\text { Körperstelle, §6 }\end{array}$ & 800 & 46,63 \\
\hline 2015 & $\begin{array}{l}\text { Anlegen einer oder mehrerer Redon- } \\
\text { drainagen }\end{array}$ & 60 & 3,50 \\
\hline 0204 & Kompressionsverband & 95 & 3,79 \\
\hline
\end{tabular}




\section{Hautreduktion und Lifting bei Ptosis (Tab. 32)}

Tab. 32

\begin{tabular}{|c|c|c|c|}
\hline Ziff. & Leistungstext & Punkte & EURO \\
\hline 2414 & Reduktionsplastik der Mamma & 2800 & 163,20 \\
\hline 2415 & $\begin{array}{l}\text { Aufbauplastik der Mamma, einschließ- } \\
\text { lich Verschiebeplastik }\end{array}$ & 2000 & 116,57 \\
\hline $2414 \mathrm{~A}$ & $\begin{array}{l}\text { Lifting des Hautmantels, entspricht } \\
\text { Reduktionsplastik Mamma, } \S 6\end{array}$ & 2800 & 163,20 \\
\hline $\begin{array}{l}2382 \\
4 \times\end{array}$ & $\begin{array}{l}\text { Schwierige Hautlappenplastik oder } \\
\text { Spalthauttransplantation }\end{array}$ & 2000 & 116,57 \\
\hline $\begin{array}{l}2394 \\
4 \times\end{array}$ & $\begin{array}{l}\text { Implantation eines Rundstiellappens, } \\
\text { einschließlich Modellierung am Ort }\end{array}$ & 2200 & 128,23 \\
\hline $\begin{array}{l}2381 \\
4 \times\end{array}$ & Einfache Hautlappenplastik & 370 & 21,57 \\
\hline 2417 & $\begin{array}{l}\text { Operative Entnahme einer Mamille und } \\
\text { intermistische Implantation an anderer } \\
\text { Körperstelle }\end{array}$ & 800 & 46,63 \\
\hline 2015 & $\begin{array}{l}\text { Anlegen einer oder mehrerer Redon- } \\
\text { drainagen }\end{array}$ & 60 & 3,50 \\
\hline 0204 & Kompressionsverband & 95 & 3,79 \\
\hline 0206 & Tape-Verband & 70 & 4,08 \\
\hline \multicolumn{4}{|c|}{ Kontralaterale Mamma: } \\
\hline 2414 & Reduktionsplastik der Mama & 2800 & 163,20 \\
\hline $2415 A$ & $\begin{array}{l}\text { Remodellierung der Restbrust, ent- } \\
\text { spricht Rekonstruktion der Restbrust, } § 6\end{array}$ & 2000 & 116,57 \\
\hline $2414 A$ & $\begin{array}{l}\text { Lifting des Hautmantels, entspricht } \\
\text { Reduktionsplastik Mamma, } \S 6\end{array}$ & 2800 & 163,20 \\
\hline $\begin{array}{l}2382 \\
4 \times\end{array}$ & $\begin{array}{l}\text { Schwierige Hautlappenplastik oder } \\
\text { Spalthauttransplantation (kranial, } \\
\text { lateral, medial, kaudal) }\end{array}$ & 739 & 43,07 \\
\hline $\begin{array}{l}2394 \\
4 \times\end{array}$ & $\begin{array}{l}\text { Implantation eines Rundstiellappens, } \\
\text { einschließlich Modellierung am Ort } \\
\text { (kranial, lateral, medial, kaudal) }\end{array}$ & 2200 & 128,23 \\
\hline $\begin{array}{l}2381 \\
4 \times\end{array}$ & $\begin{array}{l}\text { Einfache Hautlappenplastik (kranial, } \\
\text { lateral, medial, kaudal) }\end{array}$ & 370 & 18,07 \\
\hline $2417 A$ & $\begin{array}{l}\text { Rezentrierung der Mamille, entspricht } \\
\text { operative Entnahme einer Mamille und } \\
\text { intermistische Implantation an anderer } \\
\text { Körperstelle, § } 6\end{array}$ & 800 & 46,63 \\
\hline 2015 & $\begin{array}{l}\text { Anlegen einer oder mehrerer Redon- } \\
\text { drainagen }\end{array}$ & 60 & 3,50 \\
\hline 0204 & Kompressionsverband & 95 & 3,79 \\
\hline
\end{tabular}

Radikale Ablatio mamma

- Schwierige Verschiebeplastiken - lateral, medial, kranial, kaudal (Tab. 33)

Tab. 33

\begin{tabular}{|llcc}
\hline Ziff. & Leistungstext & Punkte & EURO \\
\hline 2413 & $\begin{array}{l}\text { Absetzen einer Brustdrüse mit Aus- } \\
\text { räumung der regionären Lymphstrom- } \\
\text { gebiete }\end{array}$ & 2310 & 134,64 \\
\hline 2583 & $\begin{array}{l}\text { Neurolyse des Nervus thoracicus dorsalis } \\
\text { N583 }\end{array}$ & 924 & 53,86 \\
\hline 2583 & $\begin{array}{l}\text { Neurolyse des Nervus thoracicus longus des Nervus intercosto- } \\
\text { brachialis }\end{array}$ & 924 & 53,86 \\
\hline 2382 & $\begin{array}{l}\text { Schwierige Hautlappenplastik oder } \\
\text { Spalthauttransplantation } \\
\text { (kranial, lateral, medial, kaudal) }\end{array}$ & 739,86 \\
\hline 2015 & $\begin{array}{l}\text { Anlegen einer oder mehrerer Redon- } \\
\text { drainagen }\end{array}$ & 43,07 \\
\hline 0204 & $\begin{array}{l}\text { Kompressionsverband } \\
0206\end{array}$ & Tape-Verband & 3,50 \\
\hline & & 70 & 3,79 \\
\hline
\end{tabular}

- Rekonstruktion mit Latissimus dorsi-Flap (Tab. 34)

Tab. 34

\begin{tabular}{|c|c|c|c|}
\hline Ziff. & Leistungstext & Punkte & EURO \\
\hline 2413 & $\begin{array}{l}\text { Absetzen einer Brustdrüse mit Aus- } \\
\text { räumung der regionären Lymphstrom- } \\
\text { gebiete }\end{array}$ & 2310 & 134,64 \\
\hline 2583 & Neurolyse des Nervus thoracicus dorsalis & 924 & 53,86 \\
\hline 2583 & Neurolyse des Nervus thoracicus longus & 924 & 53,86 \\
\hline 2583 & $\begin{array}{l}\text { Neurolyse des Nervus intercosto- } \\
\text { brachialis }\end{array}$ & 924 & 53,86 \\
\hline 2416 & Aufbauplastik nach Mammaamputation & 3000 & 174,86 \\
\hline $\begin{array}{l}2382 \\
4 \times\end{array}$ & $\begin{array}{l}\text { Schwierige Hautlappenplastik oder } \\
\text { Spalthauttransplantation } \\
\text { (kaudal, medial, lateral, kranial) }\end{array}$ & 739 & 43,07 \\
\hline 2070 & Muskelkanalbildung(en) & 1660 & 96,76 \\
\hline 2015 & $\begin{array}{l}\text { Anlegen einer oder mehrerer Redon- } \\
\text { drainagen }\end{array}$ & 60 & 3,50 \\
\hline 0204 & Kompressionsverband & 95 & 3,79 \\
\hline 0206 & Tape-Verband & 70 & 4,08 \\
\hline \multicolumn{4}{|c|}{ Entnahmestelle am Rücken: } \\
\hline 2083 & Freie Sehnentransplantation & 1650 & 96,17 \\
\hline 2386 & $\begin{array}{l}\text { Schleimhauttransplantation, einschließ- } \\
\text { lich operativer Unterminierung der Ent- } \\
\text { nahmestelle und plastischer Deckung }\end{array}$ & 688 & 40,10 \\
\hline $\begin{array}{l}2382 \\
4 \times\end{array}$ & $\begin{array}{l}\text { Schwierige Hautlappenplastik oder } \\
\text { Spalthauttransplantation } \\
\text { (lateral, medial, kaudal, kranial) }\end{array}$ & 739 & 43,07 \\
\hline 2015 & $\begin{array}{l}\text { Anlegen einer oder mehrerer Redon- } \\
\text { drainagen }\end{array}$ & 60 & 3,50 \\
\hline 0204 & Kompressionsverband & 95 & 3,79 \\
\hline 0206 & Tape-Verband & 70 & 4,08 \\
\hline
\end{tabular}


Tab. 35

\begin{tabular}{|c|c|c|c|}
\hline Ziff. & Leistungstext & Punkte & EURO \\
\hline 2413 & $\begin{array}{l}\text { Absetzen einer Brustdrüse mit Aus- } \\
\text { räumung der regionären Lymphstrom- } \\
\text { gebiete }\end{array}$ & 2310 & 134,64 \\
\hline 2583 & Neurolyse des Nervus thoracicus dorsalis & 924 & 53,86 \\
\hline 2583 & Neurolyse des Nervus thoracicus longus & 924 & 53,86 \\
\hline 2583 & $\begin{array}{l}\text { Neurolyse des Nervus intercosto- } \\
\text { brachialis }\end{array}$ & 924 & 53,86 \\
\hline 2416 & Aufbauplastik nach Mammaamputation & 3000 & 174,86 \\
\hline $\begin{array}{l}2382 \\
4 \times\end{array}$ & $\begin{array}{l}\text { Schwierige Hautlappenplastik oder } \\
\text { Spalthauttransplantation } \\
\text { (kranial, medial, lateral, kaudal) }\end{array}$ & 739 & 43,07 \\
\hline 2070 & Muskelkanalbildung(en) & 1660 & 96,76 \\
\hline 2015 & $\begin{array}{l}\text { Anlegen einer oder mehrerer Redon- } \\
\text { drainagen }\end{array}$ & 60 & 3,50 \\
\hline 0204 & Kompressionsverband & 95 & 3,79 \\
\hline 0206 & Tape-Verband & 70 & 4,08 \\
\hline \multicolumn{4}{|c|}{ Entnahmestelle am Rücken: } \\
\hline 0700 & Laparoskopie & 800 & 46,63 \\
\hline $5030 \mathrm{~A}$ & $\begin{array}{l}\text { Videodokumentation und Videoüber- } \\
\text { wachung, entspricht Röntgenaufnahme } \\
\text { in zwei Ebenen, §6 }\end{array}$ & 100 & 5,83 \\
\hline 2083 & Freie Sehnentransplantation & 1650 & 96,17 \\
\hline 2386 & $\begin{array}{l}\text { Schleimhauttransplantation, einschließ- } \\
\text { lich operativer Unterminierung der Ent- } \\
\text { nahmestelle und plastischer Deckung }\end{array}$ & 688 & 40,10 \\
\hline $\begin{array}{l}2382 \\
4 \times\end{array}$ & $\begin{array}{l}\text { Schwierige Hautlappenplastik oder } \\
\text { Spalthauttransplantation } \\
\text { (kranial, kaudal, lateral, medial) }\end{array}$ & 739 & 43,07 \\
\hline 2015 & $\begin{array}{l}\text { Anlegen einer oder mehrerer Redon- } \\
\text { drainagen }\end{array}$ & 60 & 3,50 \\
\hline 0204 & Kompressionsverband & 95 & 3,79 \\
\hline 0206 & Tape-Verband & 70 & 4,08 \\
\hline
\end{tabular}

Tab. 36

\begin{tabular}{|c|c|c|c|}
\hline Ziff. & Leistungstext & Punkte & EURO \\
\hline 2413 & $\begin{array}{l}\text { Absetzen einer Brustdrüse mit } \\
\text { Ausräumung der regionären Lymph- } \\
\text { stromgebiete }\end{array}$ & 2310 & 134,64 \\
\hline 2583 & Neurolyse des Nervus thoracodorsalis & 924 & 53,86 \\
\hline 2583 & Neurolyse des Nervus thoracicus longus & 924 & 53,86 \\
\hline 2583 & $\begin{array}{l}\text { Neurolyse des Nervus intercosto- } \\
\text { brachialis }\end{array}$ & 924 & 53,86 \\
\hline 2416 & Aufbauplastik nach Mammaamputation & 3000 & 174,86 \\
\hline $\begin{array}{l}2382 \\
4 \times\end{array}$ & $\begin{array}{l}\text { Schwierige Hautlappenplastik oder } \\
\text { Spalthauttransplantation } \\
\text { (kranial, lateral, medial, kaudal) }\end{array}$ & 739 & 43,07 \\
\hline $2837 \mathrm{~A}$ & $\begin{array}{l}\text { Arterielle Anastomose Arteria mamma- } \\
\text { ria interna bzw. Arteria thoracodorsalis, } \\
\text { entspricht Rekonstruktive Operation an } \\
\text { einem Viszeralgefäß, § } 6\end{array}$ & 5000 & 291,44 \\
\hline 3135 & $\begin{array}{l}\text { Eröffnung der Bauchhöhle zu } \\
\text { diagnostischen Zwecken }\end{array}$ & -1110 & $-64,70$ \\
\hline 2891 & $\begin{array}{l}\text { Rekonstruktive Operation an den } \\
\text { Körpervenen }\end{array}$ & 3000 & 174,86 \\
\hline 2015 & $\begin{array}{l}\text { Anlegen einer oder mehrerer Redon- } \\
\text { drainagen }\end{array}$ & 60 & 3,50 \\
\hline 0204 & Kompressionsverband & 95 & 3,79 \\
\hline 0206 & Tape-Verband & 70 & 4,08 \\
\hline \multicolumn{4}{|c|}{ Entnahmestelle am Bauch und Gesäß: } \\
\hline 2083 & Freie Sehnentransplantation & 1650 & 96,17 \\
\hline 2070 & Muskelkanalbildung(en) & 1660 & 96,76 \\
\hline 2386 & $\begin{array}{l}\text { Schleimhauttransplantation, einschließ- } \\
\text { lich operativer Unterminierung der Ent- } \\
\text { nahmestelle und plastischer Deckung }\end{array}$ & 688 & 40,10 \\
\hline 2808 & $\begin{array}{l}\text { Operative Entnahme einer Vene zum } \\
\text { Gefäßersatz }\end{array}$ & 400 & 23,31 \\
\hline 2807 & $\begin{array}{l}\text { Operative Entnahme einer Arterie zum } \\
\text { Gefäßersatz }\end{array}$ & 739 & 43,07 \\
\hline $3284 \mathrm{~A}$ & $\begin{array}{l}\text { Durchführung einer Abdominoplastik, } \\
\text { entspricht Operation eines Nabel- oder } \\
\text { Mittellinien- oder Bauchnarbenbruches, } \\
\text { Par. } 6\end{array}$ & 2500 & 145,72 \\
\hline $\begin{array}{l}2382 \\
4 \times\end{array}$ & $\begin{array}{l}\text { Schwierige Hautlappenplastik oder } \\
\text { Spalthauttransplantation } \\
\text { (kranial, kaudal, medial, lateral) }\end{array}$ & 739 & 43,07 \\
\hline $\begin{array}{l}2381 \\
4 \times\end{array}$ & $\begin{array}{l}\text { Einfache Hautlappenplastik } \\
\text { (kranial, kaudal, medial, lateral) }\end{array}$ & 370 & 21,57 \\
\hline 2015 & $\begin{array}{l}\text { Anlegen einer oder mehrerer Redon- } \\
\text { drainagen }\end{array}$ & 60 & 3,50 \\
\hline 0204 & Kompressionsverband & 95 & 3,79 \\
\hline 0206 & Tape-Verband & 70 & 4,08 \\
\hline
\end{tabular}


Hautsparende radikale Mastektomie

- unter aufwändigem Erhalt des Hautmantels und Latissimusdorsi-Flap (Tab. 37)

Tab. 37

\begin{tabular}{|c|c|c|c|}
\hline Ziff. & Leistungstext & Punkte & EURO \\
\hline 2413 & $\begin{array}{l}\text { Absetzen einer Brustdrüse mit Aus- } \\
\text { räumung der regionären Lymphstrom- } \\
\text { gebiete }\end{array}$ & 2310 & 134,64 \\
\hline 2583 & Neurolyse des Nervus thoracico dorsalis & 924 & 53,86 \\
\hline 2583 & Neurolyse des Nervus thoracicus longus & 924 & 53,86 \\
\hline 2583 & $\begin{array}{l}\text { Neurolyse des Nervus intercosto- } \\
\text { brachialis }\end{array}$ & 924 & 53,86 \\
\hline 2416 & Aufbauplastik nach Mammaamputation & 3000 & 174,86 \\
\hline $\begin{array}{l}2382 \\
4 \times\end{array}$ & $\begin{array}{l}\text { Schwierige Hautlappenplastik oder } \\
\text { Spalthauttransplantation } \\
\text { (kranial, lateral, medial, kaudal) }\end{array}$ & 739 & 43,07 \\
\hline $2837 \mathrm{~A}$ & $\begin{array}{l}\text { Arterielle Anastomose Arteria mamma- } \\
\text { ria interna bzw. Arteria thoracica dorsa- } \\
\text { lis, entspricht Rekonstruktiver Operation } \\
\text { an einem Viszeralgefäß, } § 6\end{array}$ & 5000 & 291,44 \\
\hline 3135 & $\begin{array}{l}\text { Eröffnung der Bauchhöhle zu } \\
\text { diagnostischen Zwecken }\end{array}$ & -1110 & $-64,70$ \\
\hline 2891 & $\begin{array}{l}\text { Rekonstruktive Operation an den } \\
\text { Körpervenen }\end{array}$ & 3000 & 174,86 \\
\hline 2015 & $\begin{array}{l}\text { Anlegen einer oder mehrerer Redon- } \\
\text { drainagen }\end{array}$ & 60 & 3,50 \\
\hline 0204 & Kompressionsverband & 95 & 3,79 \\
\hline 0206 & Tape-Verband & 70 & 4,08 \\
\hline \multicolumn{4}{|c|}{ Entnahmestelle am Bauch oder Gesäß: } \\
\hline 2083 & Freie Sehnentransplantation & 1650 & 96,17 \\
\hline 2070 & Muskelkanalbildung(en) & 1660 & 96,76 \\
\hline 2386 & $\begin{array}{l}\text { Schleimhauttransplantation, einschließ- } \\
\text { lich operativer Unterminierung der Ent- } \\
\text { nahmestelle und plastischer Deckung }\end{array}$ & 688 & 40,10 \\
\hline 2808 & $\begin{array}{l}\text { Operative Entnahme einer Vene zum } \\
\text { Gefäßersatz }\end{array}$ & 400 & 23,31 \\
\hline 2807 & $\begin{array}{l}\text { Operative Entnahme einer Arterie zum } \\
\text { Gefäßersatz }\end{array}$ & 739 & 43,07 \\
\hline $3284 \mathrm{~A}$ & $\begin{array}{l}\text { Durchführung einer Abdominoplastik, } \\
\text { entspricht Operation eines Nabel- oder } \\
\text { Mittellinien- oder Bauchnarbenbruches, } \\
\S 6\end{array}$ & 2500 & 145,72 \\
\hline $\begin{array}{l}2382 \\
4 \times\end{array}$ & $\begin{array}{l}\text { Schwierige Hautlappenplastik oder } \\
\text { Spalthauttransplantation } \\
\text { (kranial, lateral, medial, kaudal) }\end{array}$ & 739 & 43,07 \\
\hline $\begin{array}{l}2381 \\
4 \times\end{array}$ & $\begin{array}{l}\text { Einfache Hautlappenplastik } \\
\text { (kranial, lateral, medial, kaudal) }\end{array}$ & 370 & 21,57 \\
\hline 2015 & $\begin{array}{l}\text { Anlegen einer oder mehrerer Redon- } \\
\text { drainagen }\end{array}$ & 60 & 3,50 \\
\hline 0204 & Kompressionsverband & 95 & 3,79 \\
\hline 0206 & Tape-Verband & 70 & 4,08 \\
\hline
\end{tabular}

Komplexe onkoplastische Operationsverfahren beim Mammakarzinom

Klassifikation onkologischer, rekonstruktiver und onkoplastischer Operationsverfahren beim Mammakarzinom nach Komplexitätsgraden $1-6$

\section{Brusterhaltende Operationsverfahren}

\section{Einfache brusterhaltende Mammakarzinomoperationen}

(histologisch kontrollierte Tumorexzision als wide excision bis zur Quadrantektomie mit Defektdeckung über alleinige, direkte Adaptation, ohne Mobilisation von glandulärem Gewebe oder Mobilisation von Hautlappen)

\section{Komplexe brusterhaltende Mammakarzinom- operationen}

(zusätzliche intramammäre Rekonstruktion des Drüsenkörpers durch Mobilisation glandulärer Lappen subkutan oder epifaszial und ggf. Mobilisation des Hautmantels zur Defektdeckung)

\section{Onkoplastische brusterhaltende Mammakarzinom- operationen}

(Tumorexzision über komplexere Umschneidungsfiguren mit Hautresektion, einschließlich Rezentrierung des Mamillenareolakomplexes und intramammärer Defektdeckung über glanduläre Lappenplastiken)

\section{Komplexe onkoplastische brusterhaltende Mammakarzinomoperationen}

a) Tumoradaptierte Lifting-Operationen mit Desepithelialisierung und komplexen Umschneidungsfiguren (ohne weitere Resektion von Brustdrüsengewebe)

1. zentrale Stielung

2. zentral-inferiore Stielung

3. kraniale Stielung

4. free-nipple Transfer

Auswahl der Umschneidungsfigur in Abhängigkeit vom Hautüberschuss mit resultierendem Narbenbild rein periareolär, vertikal oder als inverted-T

b) Tumorresektion mit Defektdeckung über lokale Lappenplastiken mit oder ohne Hautersatz

1. Transpositionslappenplastiken (z.B. thorakoepigastrische Lappenplastik)

2. Rotationslappenplastiken

\section{Komplexe onkoplastische brusterhaltende} Mammakarzinomoperationen mit zusätzlicher Resektion (Reduktion) des Brustdrïsengewebes Tumoradaptierte Reduktionsplastik

1. zentrale Stielung

2. zentral-inferiore Stielung

3. kraniale Stielung

4. free-nipple Transfer

Auswahl der Umschneidungsfigur in Abhängigkeit vom Hautüberschuss mit resultierendem Narbenbild rein periareolär, vertikal oder als inverted-T 
6. Komplexe onkoplastische brusterhaltende Mammakarzinomoperationen mit Defektdeckung über Fernlappenplastiken

1. Tumorresektion mit partiellem Volumenersatz durch endoskopische Latissimus-Dorsi-Lappenplastik

2. Tumor- und Hautresektion mit partiellem Volumen - und Hautersatz über Latissimus-Dorsi-Lappenplastik mit Hautinsel

3. Tumor- und ggf. Hautresektion mit partiellem Volumen - und ggf. Hautersatz über gestielte TRAM-Lappenplastik

4. Tumor- und ggf. Hautresektion mit partiellem Volumen - und ggf. Hautersatz über freie Lappenplastiken mit mikrovaskulärem Gefäßanschluss (z. B. DIEP, SIEA, SGAP)

\section{Ablative Operationsverfahren}

1. Einfache ablative Mammakarzinomoperationen Modifiziert radikale Mastektomie (ohne Rekonstruktion)

\section{Komplexe ablative Mammakarzinomoperationen}

Radikale Mastektomie (mit Resektion der Pectoralismuskulatur, ohne Rekonstruktion)

\section{Onkoplastische ablative Mammakarzinomoperationen mit Prothesenrekonstruktion (oder Rekonstruktion nach Ablatio) \\ A}

1. Modifiziert radikale Mastektomie mit Implantation eines Expanders

2. Expanderexplantation und Implantation eines definitiven Implantates

3. Prothesenwechsel und Rekonfiguration bei Kapselfibrose

B

1. Hautsparende Mastektomie und Implantation eines Implantates

2. Nipple sparing Mastektomie und Implantation eines Implantates

\section{Komplexe onkoplastische ablative Mammakarzinom-} operationen mit Defektdeckung ïber lokale Lappenplastiken (auch bei ausgedehntem Rezidiv der Thoraxwand)

1. Transpositionslappenplastiken (z.B. Thorakoepigastrische Lappenplastik)

2. Rotationslappenplastiken

3. Axiale Verschiebelappenplastiken

5. Komplexe onkoplastische ablative Mammakarzinomoperationen mit Rekonstruktion oder Defektdeckung über gestielte Fernlappenplastiken

A. Latissimus-Dorsi-Lappenplastiken

1. Erweiterte Latissimus-Dorsi-Lappenplastik zur Primärrekonstruktion im Rahmen eines ablativen Operationsverfahrens (mod. Rad. Mastektomie HSM, NSM)

2. Erweiterte Latissimus-Dorsi-Lappenplastik zur Sekundärrekonstruktion nach ablativen Operationsverfahren oder zur Defektdeckung an der Thoraxwand

3. Erweiterte Latissimus-Dorsi-Lappenplastik zur autologen Konversion bei Prothesenkomplikationen
B. TRAM-Lappenplastiken

1. TRAM-Lappenplastik zur Primärrekonstruktion im Rahmen eines ablativen Operationsverfahrens (mod. Rad. Mastektomie, HSM, NSM)

2. TRAM-Lappenplastik zur Sekundärrekonstruktion nach ablativen Operationsverfahren oder zur Defektdeckung an der Thoraxwand

3. TRAM-Lappenplastik zur autologen Konversion bei Prothesenkomplikationen

6. Komplexe onkoplastische ablative Mammakarzinomoperationen mit Rekonstruktion oder Defektdeckung über freie Lappenplastiken mit mikrovaskulärem Gefäßanschluss (DIEP, SIEA, SGAP, free-TRAM, u. a.)

1. freie Lappenplastik zur Primärrekonstruktion im Rahmen eines ablativen Operationsverfahrens (mod. Rad. Mastektomie, HSM, NSM)

2. freie Lappenplastik zur Sekundärrekonstruktion nach ablativen Operationsverfahren oder zur Defektdeckung an der Thoraxwand

3. freie Lappenplastik zur autologen Konversion bei Prothesenkomplikationen

III. Detaillierte Darstellung der Operationsschritte bei komplexen onkoplastischen Mammakarzinomoperationen durch tumoradaptierte Reduktion mit Lifting bei Makromastie und Ptosis mit Segment-/Quadrantenresektion, einschließlich Nachresektaten - in der Besonderheit bei zentral inferiorer Stielung nach Hester-Bostwick

- Anzeichnen der Umschneidungsfigur für Mastopexie, Segment-/Quadrantenresektion, Reduktion, Restbrustrekonstruktion, Remodellierung und Lymphonodektomie an der stehenden wachenden Patientin mit anschließender Fotodokumentation sowohl des individuellen Situs wie der Umschneidungsfigur

- Desepithelisierung der Umschneidungsfigur

- Ablösungen des Subkutanmantels zum Bilden von 4 Verschiebelappen in allen Ebenen zur abschließenden Remodellierung des Hautmantels der Brust nach Mastopexie (kranial, kaudal, medial, lateral)

- Tumor- bzw. Segmentresektion bzw. Tumorexzisionen bei mehreren Herden und mehreren präoperativen Drahtmarkierungen mit Pectoralisfaszie und ggf. -muskulatur

- Schnellschnitte

- Nachresektate in allen Ebenen nach Maßgabe des Schnellschnittes der Aussage des Pathologen

- Bei tumoradaptierter Reduktionsmastektomie Reduktion des Drüsenfettgewebes

- Bei tumoradaptiertem Hautlifting Resektion der Hautareale zum Lifting

- Aufgrund größerer Defekte Bilden von in der Mehrzahl der Fälle 4 glandulären Rotationslappen zur glandulären Defektdeckung und Rekonstruktion der Restbrust

- Sorgfältige Hämostase und Einlage von Redondrainagen

- Rekonstruktion der Restbrust aus den 4 glandulären Rotationslappen mit entsprechenden Liftingmaßnahmen der rekonstruierten Restbrust (Liftingmaßnahmen und Liftingnähte bzw. Coriumstabilisierung) 
- Remodellierung der Brust durch die zuvor gebildeten kutanen Verschiebelappen zum spannungsfreien Hautverschluss und „trimmen“ überschüssiger Haut

- Rezentrierung der Mamille

- Kompressionsverband

- Tape-Verband

- Axilladissektion Level I- III inklusive Sentinel-Verfahren (mit Blau und Technetium)

- Angleichung kontralaterale Mamma

\section{Begründung der Komplexität am schwierigen Fallbeispiel, der tumoradaptierten Reduktionsmastektomie mit Lifting sowie axillärer Lymphonodektomie und kontralateraler Angleichung \\ Zielvorstellung der Operation}

Die o.g. Operation vereint drei verschiedene Indikationsbereiche bei besonders schwieriger Fallkonstellation unter Kombination von

1. die Ermöglichung durch die aufwändigen plastisch-operativen Verfahren der möglichst großvolumigen Resektion diffiziler Mammakarzinome, die einerseits aus invasiven Komponenten, andererseits aus sehr großen begleitenden Carcinoma in situ-Komponenten bestehen und unter Beachtung evtl. zusätzlich vorhandener ungünstiger Tumorlokalisation, die großzügige Resektion unter leitliniengerechter Erzielung von freien Resektionsrändern (free margins) unter Vermeidung einer sonst typischerweise iatrogen verursachten Defektbildung.

2. die radioonkologisch sinnvolle Verkleinerung des Fettdrüsengewebes bei Makromastie inkl. der Liftingoperation der extrem hängenden Brust zur Optimierung der postoperativen Bestrahlung unter Minimierung der Strahlenkomplikationen, zur Verminderung der Strahlendermatitis, der gewebespezifischen Begleitreaktionen inkl. Langzeitfolgen wie Fettgewebsnekrose, chronische Schmerzproblematik usw.;

3. insbesondere auch die Vermeidung von Asymmetrien mit konsekutiven Einschränkungen der Lebensqualität sowie statische und orthopädische Beschwerdekomplexe

4. der medizinisch notwendigen Verkleinerungsoperation bei Makromastie unter Reduktion des Fettdrüsengewebes in für die Lebensqualität der Patientin notwendiger Volumengröße

\section{Wichtigste Operationsschritte entsprechend der Komplexitätsgrade}

- Bilden der Hautfettlappen zwei nach kranial, zwei nach kaudal

- Großzügige Segmentresektion bzw. Quadrantenresektion des Tumors unter Umständen mit Hautspindel

- Nachresektate nach allen Ebenen sowie nach ventral und nach dorsal ggf. unter Mitnahme der angrenzenden Pektoralisfaszie bzw. der angrenzenden Pektoralismuskulatur

- Bildung von vier glandulären Rotationslappen, um bei ausgeprägter Defektbildung durch die vorherige großvolumige Tumorexzision unter Vermeidung von Hohlraumbildungen decken zu können und eine Rekonstruierung einer neuen Brust zu ermöglichen

- Reduktionsplastik der Mamma im Sinne der Resektion allen überschüssigen Drüsengewebes und Fettgewebes, um das angestrebte neue Restvolumen der Brust im onkologischen, kosmetischen und radioonkologischen Sinne erzielen zu können
- Resektion des überschüssigen Hautmantels zur Erzielung des Liftings, einerseits im Sinne des straffen Hautmantels zur Defektdeckung, andererseits, die Korrektur der Ptosis der Brust in o.g. Sinne zu ermöglichen

- Bildung des Stieles für den Mamillenareolakomplex zur Ermöglichung der optimalen Durchblutung von Mamille und Areola durch Bildung eines zentrokaudalen oder je nach Technik anderen Gefäßstiels für die Mamille (oder falls nötig durch freien Mamillentransfer)

- In Ergänzung der Stielung werden die vier glandulären Rotationslappen zur Reformierung der neuen Brust aneinander angepasst, ggf. neu geformt, fixiert, modelliert und eingepasst (3. Abrechnung nach 2415)

Dieser Schritt entspricht der Reformierung

- durch Aufhängung des Gefäßstieles aus Bündelung der Rotationslappen an der Thoraxwand folgt eine Remodellierung der neuen Brust im Hinblick auf Höhe, Symmetrie und Formgebung;

- die neu gebildete Drüsenkörperbrust wird nun über das Anpassen der Hautfettlappen mit einer neuen Hautfetthülle überzogen und durch das Modellieren des Hautmantels mit erneuter Hautresektion, Lifting und Fixierung zur oberflächlichen Formgebung und Fixierung rekonstruiert.

Erst nach Bildung des neuen Hautmantels mit Fixierung erfolgt dann die Einpassung des Mamillenareolakomplexes durch Einpassung in den neuen Hautmantel im Sinne der Neupositionierung und Zentrierung der Mamille bzw. des Mamillenareolakomplexes.

Bei dem genannten Operationsverfahren handelt es sich um ein auf hochspezialisierte Brustzentren beschränktes spezielles innovatives und einzigartiges Operationsverfahren. Neben den genannten Zielvorstellungen wird damit durch komplexe onkochirurgische Rekonstruktionsverfahren eine Ablatio vermieden, darüber hinaus eine Optimierung der Lebensqualität der Patientin erzielt und dies ohne zusätzlich erforderliche Fernlappenplastik, welche mit erheblich höherem Trauma für die Patientin einhergeht bzw. unter Vermeidung des Einlegens einer Prothese mit allen negativen Folgeerscheinungen für die Patientin.

Die Komplexität des geschilderten Eingriffes erklärt sich aus der Tatsache, dass vier verschiedene Operationsverfahren in einer Operation zusammengefasst sind.

Demgegenüber steht beim ablativen Operationsverfahren neben dem Trauma (Postmastektomie-Syndrom) für die Patientin die Notwendigkeit der Rekonstruktion in für gewöhnlich mehreren weiteren Operationen mit ungleich höherer Belastung und ungleich höherem Aufwand für die Patientin.

Bei der Fernlappenplastik ergibt sich neben dem ungleich schwerwiegenderen Trauma für die Patientin häufig die Notwendigkeit von zwei Operationen, wie der Delay-Operation vor Fernlappenplastik im Sinne eines TRAM-Flaps. Bei der einzeitigen oder zweizeitigen prothetischen Versorgung muss der Hautmantel nach Ablatio mammae erst durch eine Expanderprothese aufgedehnt werden und in einem weiteren Eingriff die definitive Prothese eingelegt werden mit allen Folgeerscheinungen, wie 
z.B. Kapselkontrakturen und notwendigen Korrektureingriffen im Laufe des postoperativen Verlaufs bzw. des Lebens der Patientin.

So werden bei Fernlappenplastiken häufig zwei weitere Eingriffe notwendig, die durch Einsatz o.g. Techniken vermieden werden können.

Bei der prothetischen Versorgung ebenfalls zwei zusätzliche Eingriffe, nicht eingerechnet die durch Kapselbildung notwendigen Sekundäreingriffe und Komplikationen.

Die Voraussetzungen für diese komplexen Operationsverfahren sind die Zentralisierung in großen interdisziplinären Zentren mit komplettem operativ-senologischem Know-how und der operativen Infrastruktur.

Zusätzlich erforderlich sind spezielle Weiterbildungen, wie z.B. Schwerpunkt operative Gynäkologie und Onkologie oder plastische Mammachirurgie, um den entsprechenden Anforderungen gerecht zu werden.

\section{Senologie}

Sonographische Markierung des Befundes (Tab. 38)

\section{Tab. $\mathbf{3 8}$}

\begin{tabular}{cllc}
\hline Ziffer & Leistungstext & Punkte & Euro \\
\hline 0418 & $\begin{array}{l}\text { Ultraschalluntersuchung einer } \\
\text { Brustdrüse }\end{array}$ & 0210 & 12,24 \\
\hline $1251 \mathrm{~A}$ & $\begin{array}{l}\text { Markierung des Befundes entspricht } \\
\text { Lokalisation einer Netzhautveränderung } \\
\text { als Voraussetzung für einen gezielten } \\
\text { intraokularen Eingriff, §6 }\end{array}$ & 0273 & 15,91 \\
\hline
\end{tabular}

Sonographische Drahtmarkierung des Befundes (Tab. 39)

$$
\text { Tab. } 39
$$

\begin{tabular}{|c|c|c|c|}
\hline Ziffer & Leistungstext & Punkte & Euro \\
\hline 0418 & $\begin{array}{l}\text { Ultraschalluntersuchung einer } \\
\text { Brustdrüse }\end{array}$ & 0210 & 12,24 \\
\hline 0490 & Infiltrationsanästhesie kleiner Bezirke & 0061 & 3,56 \\
\hline 0314 & Punktion der Mamma & 0120 & 6,99 \\
\hline $1251 \mathrm{~A}$ & $\begin{array}{l}\text { Markierung des Befundes entspricht } \\
\text { Lokalisation einer Netzhautveränderung } \\
\text { als Voraussetzung für einen gezielten } \\
\text { intraokularen Eingriff, § } 6\end{array}$ & 0273 & 15,91 \\
\hline
\end{tabular}

Sentinel - Markierung (Tab. 40)

Tab. 40

\begin{tabular}{|lllc}
\hline Ziffer & Leistungstext & Punkte & Euro \\
\hline 0321 & $\begin{array}{l}\text { Untersuchung von natülichen Gängen } \\
\text { oder Fisteln mittels Sonde }\end{array}$ & 0200 & 11,66 \\
\hline 0370 & $\begin{array}{l}\text { Einbringung des Kontrastmittels zur } \\
\text { Darstellung natürlicher, künstlicher oder } \\
\text { krankhaft entstandener Gänge, Gang- } \\
\text { systeme, Hohlräume oder Fisteln - } \\
\text { ggf. intraoperativ }\end{array}$ & \\
\hline 5430 & $\begin{array}{l}\text { Tumorszintigraphie mit radioaktiv mar- } \\
\text { kierten unspezifischen Tumormarkern - } \\
\text { einer Region }\end{array}$ & 11,66 \\
\hline
\end{tabular}

\section{Geburtshilfe}

\section{Pränataldiagnostik}

1. Ultraschall Nackenfalte beim Feten (Tab. 41)

Tab. 41

\begin{tabular}{lllc}
\hline Ziffer & Leistungstext & Punkte & Euro \\
\hline 0415 & $\begin{array}{l}\text { Ultraschalluntersuchungen im Rahmen } \\
\text { der Mutterschaftsvorsorge }\end{array}$ & 0300 & 17,49 \\
\hline
\end{tabular}

\section{- Ziffer 415 GOÄ zum 3,5fach Faktor}

Begründung: Erhöhter Schwierigkeitsgrad und Zeitaufwand bei frühem Gestationsalter und bei zusätzlich durchgeführter Messung der fetalen Nackentransparenz.

Durchführung möglich erst ab: 11/0 bis 13/6 SSW

Medizinische Indikation: Die fetale Nackentransparenzmessung dient zur Risikoerfassung von Karyotypanomalien und fetalen Fehlbildungen.

\section{Fehlbildungsultraschall (Tab. 42)}

- Voraussetzungen

- Degum II oder

- Spezielle Geburtshilfe und Perinatalmedizin oder

- Fachkunde Sonographie des Fetus in der Frauenheilkunde

- Gerätenachweis (zusätzlich!)

Durchführung möglich erst ab: 14/0 SSW

Medizinische Indikation: Zur gezielten Ausschlussdiagnostik einer fetalen Fehlbildung bis zu dreimal im gesamten Schwangerschaftsverlauf, im Positivfall einer fetalen Fehlbildung oder Erkrankung auch häufiger. Grundsätzlich sind alle sonographisch nachweisbaren fetalen Organsysteme zu erfassen. Gilt entsprechend Ic zu Abschnitt B. Nr. 4 der Mutterschafts-Richtlinien in der jeweils geltenden Fassung. 
Tab. 42

\begin{tabular}{|c|c|c|c|}
\hline Ziffer & Leistungstext & Punkte & Euro \\
\hline $1006 A$ & $\begin{array}{l}\text { Gezielte weiterführende differenzial- } \\
\text { diagnostische sonographische Abklä- } \\
\text { rung bei auf Grund einer Untersuchung } \\
\text { nach Nr. } 415 \text { erhobenem Verdacht auf } \\
\text { Schädigung eines Feten durch Fehlbil- } \\
\text { dung oder Erkrankung oder ausgewiese- } \\
\text { ner besonderer Risikosituation (Genetik, } \\
\text { Anamnese, exogene Noxe) unter Ver- } \\
\text { wendung eines Ultraschalluntersu- } \\
\text { chungsgerätes, dass mindestens über } 64 \\
\text { Kanäle im Sende- und Empfangsbereich, } \\
\text { eine variable Tiefenfokussierung mindes- } \\
\text { tens } 64 \text { Graustufen und eine aktive Ver- } \\
\text { größerungsmöglichkeit für Detailldar- } \\
\text { stellungen verfügt. Ggf. mehrfach - zur } \\
\text { gezielten Ausschlussdiagnostik bis zu } \\
\text { dreimal im gesamten SS-Verlauf im Po- } \\
\text { sitivfall einer fetalen Fehlbildung oder } \\
\text { Erkrankung auch häufiger, gilt entspre- } \\
\text { chend Anlage Ic zu Abschnitt B. Nr. } 4 \text { der } \\
\text { Mutterschaftsrichtlinien in der jeweils } \\
\text { geltenden Fassung } \\
\text { pro Fet berechnungsfähig } \\
\text { Analog Nr. } 5373\end{array}$ & 1900 & 110,75 \\
\hline
\end{tabular}

3. Fetale Echokardiographie beim Feten (Tab. 43)

- Voraussetzungen

- Degum II oder

- Spezielle Geburtshilfe und Perinatalmedizin oder

- Fachkunde Sonographie des Fetus in der Frauenheilkunde

- Gerätenachweis (zusätzlich!)

Durchführung möglich erst ab: 11/0 SSW

Tab. 43

\begin{tabular}{|c|c|c|c|}
\hline Ziffer & Leistungstext & Punkte & Euro \\
\hline \multirow[t]{4}{*}{$1007 \mathrm{~A}$} & $\begin{array}{l}\text { Dreidimensionale und/oder farbkodierte } \\
\text { Dopplerechokardiographische Untersu- } \\
\text { chung eines Feten einschließlich Bilddo- } \\
\text { kumentation, einschließlich Doppler- } \\
\text { echokardiographische Untersuchung } \\
\text { ggf. einschließlich Untersuchung mit } \\
\text { cw-Doppler und Fequenzspektrumana- } \\
\text { lyse ggf. einschließlich zweidimensiona- } \\
\text { ler echokardiographischer Untersuchung } \\
\text { mittels Time-Motion-Verfahren (M-Mo- } \\
\text { de), ggf. zusätzlich zur Leistung nach } \\
\text { Nr. A } 1006 \\
\text { gilt entsprechend Anlage } 1 \text { d zu Ab- } \\
\text { schnitt B Nr. } 4 \text { der Mutterschaftsricht- } \\
\text { linien in der jeweils geltenden Fassung }\end{array}$ & & \\
\hline & Analog Nr. 424, & 700 & 40,80 \\
\hline & Nr. 404, (Einfachsatz) & 250 & 14,57 \\
\hline & Nr. 406, (Einfachsatz) & 200 & 11,66 \\
\hline
\end{tabular}

Medizinische Indikation: Zur gezielten Ausschlussdiagnostik einer fetalen Erkrankung bzw. einer Anomalie des Herz-, Thoraxund Gefäßsystems bis zu dreimal im gesamten Schwangerschaftsverlauf, im Positivfall einer fetalen Anomalie oder Erkrankung auch häufiger. Gilt entsprechend Id zu Abschnitt B. Nr. 4 der Mutterschafts-Richtlinien in der jeweils geltenden Fassung.

\section{Doppler-Untersuchung des fetomaternalen Gefäßsystems (Tab. 44)}

Tab. $\mathbf{4 4}$

\begin{tabular}{|c|c|c|c|}
\hline Ziffer & Leistungstext & Punkte & Euro \\
\hline \multirow[t]{2}{*}{$1008 \mathrm{~A}$} & $\begin{array}{l}\text { Weiterführende differenzialdiagnosti- } \\
\text { sche sonographische Abklärung des fe- } \\
\text { tomaternalen Gefäßsystems mittels } \\
\text { Duplexverfahren, ggf. farbkodiert und/ } \\
\text { oder direktionale Dopplersonographi- } \\
\text { sche Untersuchung im fetomaternalen } \\
\text { Gefäßsystem, einschließlich Frequenz- } \\
\text { Spektrumanalyse } \\
\text { Ggfs. zusätzlich zu den Untersuchungen } \\
\text { nach den Nrn. } 415 \text { oder } 1006 \text { A } \\
\text { Gilt entsprechend Anlage } 1 \mathrm{~d} \text { zu Ab- } \\
\text { schnitt B Nr. } 4 \text { der Mutterschafts-Richt- } \\
\text { linien in der jeweils geltenden Fassung }\end{array}$ & & \\
\hline & Analog Nr. 649 & 0650 & 37,89 \\
\hline
\end{tabular}

- Voraussetzungen

- Degum II oder

- Spezielle Geburtshilfe und Perinatalmedizin oder

- Fachkunde Sonographie des Fetus in der Frauenheilkunde

- Gerätenachweis (zusätzlich!)

Durchführung möglich erst ab: 11/0 SSW

Medizinische Indikation für die 1. Untersuchung: EBM - Richtlinien allgemein

Zur Erfassung bzw. zum Ausschluss von gefäßrelevanten mütterlichen und fetalen Erkrankungen.

Medizinische Indikation der Häufigkeit der Untersuchung:

- zur Früherkennung von Erkrankung des mütterlichen und fetalen Gefäßsystems

- zur Früherkennung eines fetalen Herzfehlers bzw. fetalen Erkrankung

- bei Zustand nach IUGR, totes Kind

- bei SGA/IUGR

- bei Auffälligkeiten der fetalen Herzfrequenz

- bei Mehrlingen

- bei SIH, Präeklampsie

- bei Zustand nach SIH, Präeklampsie 


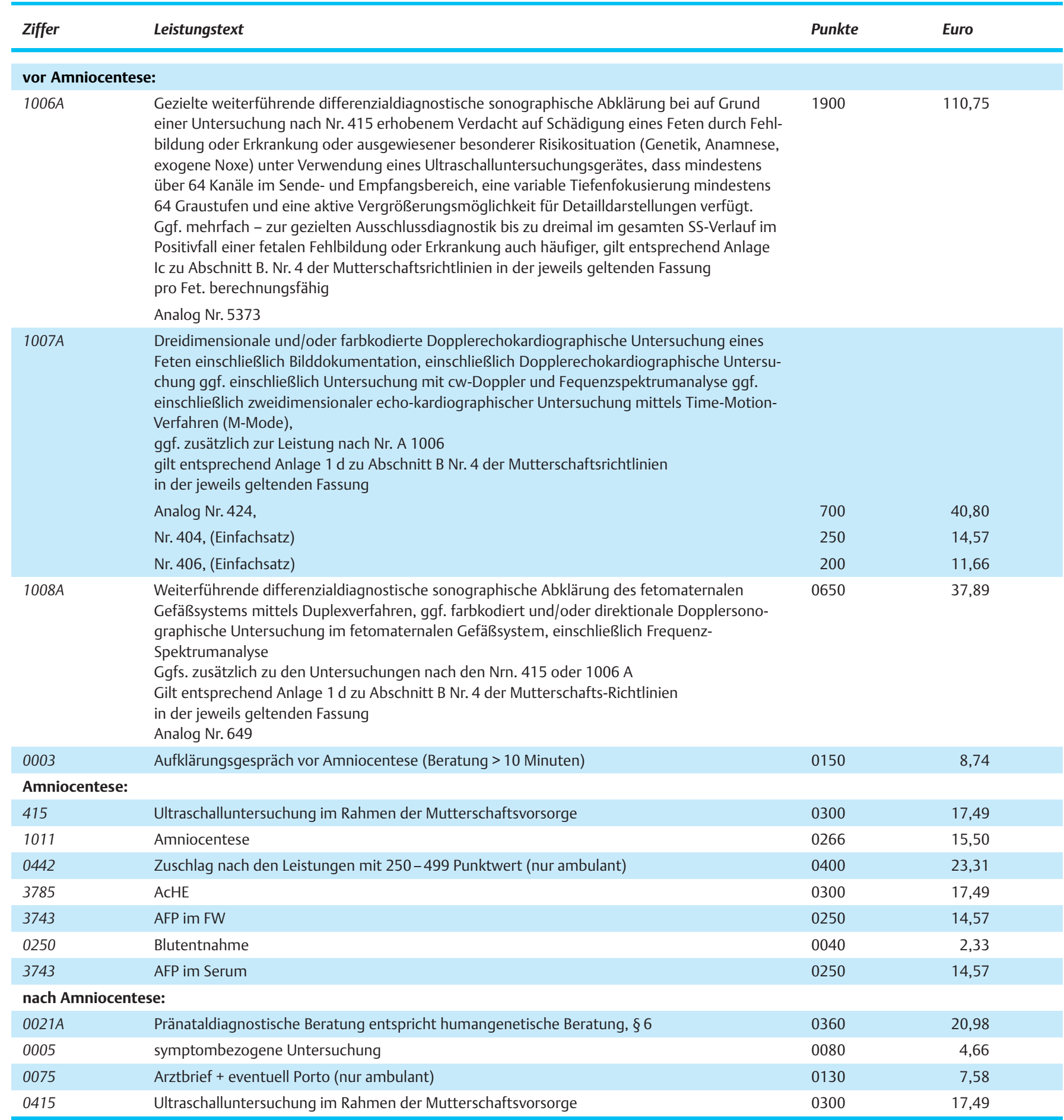

\author{
5. Invasive Diagnostik im Bereich der Pränataldiagnostik \\ - Amniocentese (Tab. 45) \\ - Voraussetzungen \\ - Degum II oder \\ - Spezielle Geburtshilfe und Perinatalmedizin oder \\ - Fachkunde Sonographie des Fetus in der Frauenheilkunde \\ - Gerätenachweis (zusätzlich!)
}

Durchführung möglich erst ab: 13/+0 SSW 
Tab. 46

\begin{tabular}{|c|c|c|c|}
\hline Ziffer & Leistungstext & Punkte & Euro \\
\hline \multicolumn{4}{|c|}{ vor Fetalblutentnahme: } \\
\hline $1006 \mathrm{~A}$ & $\begin{array}{l}\text { Gezielte weiterführende differenzialdiagnostische sonographische Abklärung bei auf Grund einer Untersuchung } \\
\text { nach Nr. } 415 \text { erhobenem Verdacht auf Schädigung eines Feten durch Fehlbildung oder Erkrankung oder aus- } \\
\text { gewiesener besonderer Risikosituation (Genetik, Anamnese, exogene Noxe) unter Verwendung eines Ultra- } \\
\text { schalluntersuchungsgerätes, dass mindestens über } 64 \text { Kanäle im Sende- und Empfangsbereich, eine variable } \\
\text { Tiefenfokusierung mindestens } \\
64 \text { Graustufen und eine aktive Vergrößerungsmöglichkeit für Detailldarstellungen verfügt. } \\
\text { Ggf. mehrfach - zur gezielten Ausschlussdiagnostik bis zu dreimal im gesamten SS-Verlauf im Positivfall } \\
\text { einer fetalen Fehlbildung oder Erkrankung auch häufiger, gilt entsprechend Anlage Ic zu Abschnitt B. Nr. } 4 \\
\text { der Mutterschaftsrichtlinien in der jeweils geltenden Fassung pro Fet berechnungsfähig } \\
\text { Analog Nr. } 5373\end{array}$ & 1900 & 110,75 \\
\hline \multirow[t]{4}{*}{$1007 A$} & $\begin{array}{l}\text { Dreidimensionale und/oder farbkodierte Dopplerechokardiographische Untersuchung eines Feten einschließlich } \\
\text { Bilddokumentation, einschließlich Doppler-echo-kardiographische Untersuchung ggf. einschließlich Unter- } \\
\text { suchung mit cw-Doppler und Fequenzspektrumanalyse ggf. einschließlich zweidimensionaler echo-kardio- } \\
\text { graphischer Untersuchung mittels Time-Motion-Verfahren (M-Mode), } \\
\text { ggf. zusätzlich zur Leistung nach Nr. A } 1006 \\
\text { gilt entsprechend Anlage } 1 \mathrm{~d} \text { zu Abschnitt B Nr. } 4 \text { der Mutterschaftsrichtlinien } \\
\text { in der jeweils geltenden Fassung }\end{array}$ & & \\
\hline & Analog Nr. 424, & 700 & 40,80 \\
\hline & Nr. 404, (Einfachsatz) & 250 & 14,57 \\
\hline & Nr. 406, (Einfachsatz) & 200 & 11,66 \\
\hline $1008 \mathrm{~A}$ & $\begin{array}{l}\text { Weiterführende differenzialdiagnostische sonographische Abklärung des fetomaternalen Gefäßsystems } \\
\text { mittels Duplexverfahren, ggf. farbkodiert und/oder direktionale Dopplersonographische Untersuchung im } \\
\text { fetomaternalen Gefäßsystem, einschließlich Frequenz-Spektrumanalyse } \\
\text { Ggfs. zusätzlich zu den Untersuchungen nach den Nrn. } 415 \text { oder } 1006 \text { A } \\
\text { Gilt entsprechend Anlage } 1 \mathrm{~d} \text { zu Abschnitt B Nr. } 4 \text { der Mutterschafts-Richtlinien } \\
\text { in der jeweils geltenden Fassung } \\
\text { Analog Nr. } 649\end{array}$ & 0650 & 37,89 \\
\hline 0003 & Aufklärungsgespräch vor FBS (Beratung > 10 Minuten) & 0150 & 8,74 \\
\hline \multicolumn{4}{|c|}{ Fetalblutentnahme } \\
\hline $1158 \mathrm{~A}$ & Analog: Fetalblutentnahme unter Ultraschallsicht entspricht Kuldoskopie, § 6 & 0739 & 43,07 \\
\hline 0443 & Zuschlag nach den Leistungen mit 500 - 799 Punktwert (nur ambulant) & 0750 & 23,31 \\
\hline 1011 & $\begin{array}{l}\text { Amniocentese } \\
*\end{array}$ & 0266 & 15,50 \\
\hline \multicolumn{4}{|c|}{ nach FBS } \\
\hline $0021 \mathrm{~A}$ & Pränataldiagnostische Beratung entspricht humangenetische Beratung, §6 & 0360 & 20,98 \\
\hline 0005 & symptombezogene Untersuchung & 0080 & 4,66 \\
\hline 0075 & Arztbrief + eventuell Porto (nur ambulant) & 0130 & 7,58 \\
\hline 0415 & Ultraschalluntersuchung im Rahmen der Mutterschaftsvorsorge & 0300 & 17,49 \\
\hline
\end{tabular}

* ggfs. zusätzliche Berechnung der Laborleistungen

- Fetalblutentnahme (Tab. 46)

- Voraussetzungen

- Degum II oder

- Spezielle Geburtshilfe und Perinatalmedizin oder

- Fachkunde Sonographie des Fetus in der Frauenheilkunde

- Gerätenachweis (zusätzlich!) 
Tab. $\mathbf{4 7}$

\begin{tabular}{|c|c|c|c|}
\hline Ziffer & Leistungstext & Punkte & Euro \\
\hline \multicolumn{4}{|c|}{ vor Chorionzottenbiopsie } \\
\hline $1006 \mathrm{~A}$ & $\begin{array}{l}\text { Gezielte weiterführende differenzialdiagnostische sono-graphische Abklärung bei auf Grund einer Untersuchung } \\
\text { nach Nr. } 415 \text { erhobenem Verdacht auf Schädigung eines Feten durch Fehlbildung oder Erkrankung oder ausge- } \\
\text { wiesener besonderer Risikosituation (Genetik, Anamnese, exogene Noxe) unter Verwendung eines Ultraschall- } \\
\text { untersuchungsgerätes, dass mindestens über } 64 \text { Kanäle im Sende- und Empfangsbereich, eine variable Tiefen- } \\
\text { fokussierung mindestens } 64 \text { Graustufen und eine aktive Vergrößerungsmöglichkeit für Detailldarstellungen ver- } \\
\text { fügt. Ggf. mehrfach - zur gezielten Ausschlussdiagnostik bis zu dreimal im gesamten SS-Verlauf im Positivfall } \\
\text { einer fetalen Fehlbildung oder Erkrankung auch häufiger, gilt entsprechend Anlage Ic zu Abschnitt B. Nr. } 4 \text { der } \\
\text { Mutterschaftsrichtlinien in der jeweils geltenden Fassung pro Fet berechnungsfähig } \\
\text { Analog Nr. } 5373\end{array}$ & 1900 & 110,75 \\
\hline \multirow[t]{4}{*}{$1007 A$} & $\begin{array}{l}\text { Dreidimensionale und/oder farbkodierte Dopplerechokardiographische Untersuchung eines Feten einschließlich } \\
\text { Bilddokumentation, einschließlich Dopplerechokardiographische Untersuchung ggf. einschließlich Untersu- } \\
\text { chung mit cw-Doppler und Fequenzspektrumanalyse ggf. einschließlich zweidimensionaler echokardiographi- } \\
\text { scher Untersuchung mittels Time-Motion-Verfahren (M-Mode), ggf. zusätzlich zur Leistung nach Nr. A } 1006 \\
\text { gilt entsprechend Anlage } 1 \mathrm{~d} \text { zu Abschnitt B Nr. } 4 \text { der Mutterschaftsrichtlinien } \\
\text { in der jeweils geltenden Fassung }\end{array}$ & & \\
\hline & Analog Nr. 424, & 700 & 40,80 \\
\hline & Nr. 404, (Einfachsatz) & 250 & 14,57 \\
\hline & Nr. 406, (Einfachsatz) & 200 & 11,66 \\
\hline $1008 \mathrm{~A}$ & $\begin{array}{l}\text { Weiterführende differenzialdiagnostische sonographische Abklärung des fetomaternalen Gefäßsystems } \\
\text { mittels Duplexverfahren, ggf. farbkodiert und/oder direktionale Dopplersonographische Untersuchung im } \\
\text { fetomaternalen Gefäßsystem, einschließlich Frequenz-Spektrumanalyse } \\
\text { Ggfs. zusätzlich zu den Untersuchungen nach den Nrn. } 415 \text { oder } 1006 \text { A } \\
\text { Gilt entsprechend Anlage } 1 \mathrm{~d} \text { zu Abschnitt B Nr. } 4 \text { der Mutterschafts-Richtlinien in der jeweils geltenden Fassung } \\
\text { Analog Nr. } 649\end{array}$ & 0650 & 37,89 \\
\hline 0003 & Aufklärungsgespräch vor CVS (Beratung > 10 Minuten) & 0150 & 8,74 \\
\hline \multicolumn{4}{|c|}{ Chorionzottenbiopsie } \\
\hline 1157 & $\begin{array}{l}\text { Chorionzottenbiopsie, transvaginal oder transabdominal unter Ultraschallsicht Analog } \\
\text { nach Nr. } 1158\end{array}$ & 0739 & 43,07 \\
\hline $4711^{\circ}$ & $\begin{array}{l}\text { Analog: Quantitative und Qualitative Differenzierung der Zottenprobe entspricht lichtmikroskopische } \\
\text { Untersuchung zum Nachweis von Pilzen im Nativmaterial nach Präparation, § } 6\end{array}$ & 0120 & 6,99 \\
\hline \multicolumn{4}{|c|}{ nach CVS } \\
\hline $0021 A$ & Pränataldiagnostische Beratung entspricht humangenetische Beratung, § 6 & 0360 & 20,98 \\
\hline 0005 & symptombezogene Untersuchung & 0080 & 4,66 \\
\hline 0075 & Arztbrief + eventuell Porto (nur ambulant) & 0130 & 7,58 \\
\hline 0415 & Ultraschalluntersuchung im Rahmen der Mutterschaftsvorsorge & 0300 & 17,49 \\
\hline
\end{tabular}

- Chorionzottenbiopsie (Tab. 47)

- Voraussetzungen

- Degum II oder

- Spezielle Geburtshilfe und Perinatalmedizin oder

- Fachkunde Sonographie des Fetus in der Frauenheilkunde

- Gerätenachweis (zusätzlich!)

Durchführung möglich erst ab: 9/+0 SSW 


\begin{tabular}{|c|c|c|c|}
\hline Ziffer & Leistungstext & Punkte & Euro \\
\hline \multicolumn{4}{|c|}{ vor intrauterinem Eingriff } \\
\hline $1006 \mathrm{~A}$ & $\begin{array}{l}\text { Gezielte weiterführende differenzialdiagnostische sonographische Abklärung bei auf Grund einer Untersuchung } \\
\text { nach Nr. } 415 \text { erhobenem Verdacht auf Schädigung eines Feten durch Fehlbildung oder Erkrankung oder ausge- } \\
\text { wiesener besonderer Risikosituation (Genetik, Anamnese, exogene Noxe) unter Verwendung eines Ultraschall- } \\
\text { untersuchungsgerätes, dass mindestens über } 64 \text { Kanäle im Sende- und Empfangsbereich, eine variable Tiefen- } \\
\text { fokusierung mindestens } 64 \text { Graustufen und eine aktive Vergrößerungsmöglichkeit für Detailldarstellungen ver- } \\
\text { fügt. Ggf. mehrfach - zur gezielten Ausschlussdiagnostik bis zu dreimal im gesamten SS-Verlauf im Positivfall } \\
\text { einer fetalen Fehlbildung oder Erkrankung auch häufiger, gilt entsprechend Anlage Ic zu Abschnitt B. Nr. } 4 \text { der } \\
\text { Mutterschaftsrichtlinien in der jeweils geltenden Fassung pro Fet berechnungsfähig } \\
\text { Analog Nr. } 5373\end{array}$ & 1900 & 110,75 \\
\hline \multirow[t]{4}{*}{$1007 A$} & $\begin{array}{l}\text { Dreidimensionale und/oder farbkodierte Dopplerechokardiographische Untersuchung eines Feten einschließlich } \\
\text { Bilddokumentation, einschließlich Doppler-echo-kardiographische Untersuchung ggf. einschließlich Untersu- } \\
\text { chung mit cw-Doppler und Fequenzspektrumanalyse ggf. einschließlich zweidimensionaler echokardiographi- } \\
\text { scher Untersuchung mittels Time-Motion-Verfahren (M-Mode), } \\
\text { ggf. zusätzlich zur Leistung nach Nr. A } 1006 \\
\text { gilt entsprechend Anlage } 1 \mathrm{~d} \text { zu Abschnitt B Nr. } 4 \text { der Mutterschaftsrichtlinien in der jeweils geltenden Fassung }\end{array}$ & & \\
\hline & Analog Nr. 424, & 700 & 40,80 \\
\hline & Nr. 404, (Einfachsatz) & 250 & 14,57 \\
\hline & Nr. 406, (Einfachsatz) & 200 & 11,66 \\
\hline $1008 \mathrm{~A}$ & $\begin{array}{l}\text { Weiterführende differenzialdiagnostische sonographische Abklärung des fetomaternalen Gefäßsystems } \\
\text { mittels Duplexverfahren, ggf. farbkodiert und/oder direktionale Dopplersonographische Untersuchung im }\end{array}$ & 0650 & 37,89 \\
\hline
\end{tabular}
mittels Duplexverfahren, ggf. farbkodiert und/oder direktionale Dopplersonographische Untersuchung im fetomaternalen Gefäßsystem, einschließlich Frequenz-Spektrumanalyse Ggfs. zusätzlich zu den Untersuchungen nach den Nrn. 415 oder 1006 A Gilt entsprechend Anlage $1 \mathrm{~d}$ zu Abschnitt B Nr. 4 der Mutterschafts-Richtlinien in der jeweils geltenden Fassung Analog Nr. 649

\begin{tabular}{|c|c|c|c|}
\hline 0003 & Aufklärungsgespräch vor IUT/IUTT (Beratung > 10 Minuten) & 0150 & 8,74 \\
\hline \multicolumn{4}{|c|}{ vor IUT/IUTT } \\
\hline $1158 \mathrm{~A}$ & Analog: Fetalblutentnahme unter Ultraschallsicht entspricht Kuldoskopie, § 6 & 0739 & 43,07 \\
\hline 0443 & Zuschlag nach den Leistungen mit 500 - 799 Punktwert (nur ambulant) & 0750 & 23,31 \\
\hline \multicolumn{4}{|c|}{ IUT/IUTT } \\
\hline $1156 A$ & Analog: Intrauterine Transfusion unter Ultraschallsicht entspricht operative Pelviskopie, §6 & 1050 & 61,20 \\
\hline 0444 & $\begin{array}{l}\text { Zuschlag nach den Leistungen mit 800-1199 Punktwert (nur ambulant) } \\
*\end{array}$ & 1300 & 75,77 \\
\hline \multicolumn{4}{|c|}{ nach IUT/IUTT } \\
\hline $1158 \mathrm{~A}$ & Analog: Fetalblutentnahme unter Ultraschallsicht entspricht Kuldoskopie, § 6 & 0739 & 43,07 \\
\hline 0443 & $\begin{array}{l}\text { Zuschlag nach den Leistungen mit 500 - } 799 \text { Punktwert (nur ambulant) } \\
* *\end{array}$ & 0750 & 23,31 \\
\hline \multicolumn{4}{|c|}{ nach intrauterinem Eingriff } \\
\hline 0448 & $\begin{array}{l}\text { Beobachtung und Betreuung eines Kranken über mehr als } 2 \text { Stunden während der Aufwach- und/oder } \\
\text { Erholungszeit }\end{array}$ & 600 & 34,97 \\
\hline $0021 A$ & Pränataldiagnostische Beratung entspricht humangenetische Beratung, §6 & 0360 & 20,98 \\
\hline 0005 & symptombezogene Untersuchung & 0080 & 4,66 \\
\hline 0075 & Arztbrief + eventuell Porto (nur ambulant) & 0130 & 7,58 \\
\hline 0415 & Ultraschalluntersuchung im Rahmen der Mutterschaftsvorsorge & 0300 & 17,49 \\
\hline
\end{tabular}

* ggfs. zusätzliche Berechnung der Laborleistungen

** ggfs. zusätzliche Berechnung der Laborleistungen

\author{
IUT/IUTT (Tab. 48) \\ Voraussetzungen \\ - Degum II oder \\ - Spezielle Geburtshilfe und Perinatalmedizin oder \\ - Fachkunde Sonographie des Fetus in der Frauenheilkunde \\ - Gerätenachweis (zusätzlich!)
}


Untersuchung 28. SSW: Sonographie

- Fetometrie und Doppler, FW-Menge

Tab. 49

\begin{tabular}{|c|c|c|c|}
\hline Ziffer & Leistungstext & Punkte & Euro \\
\hline $5377 A$ & $\begin{array}{l}\text { analog: Zuschlag für computerge- } \\
\text { steuerte Analyse und Abbildung des CTG } \\
\text { entspricht Zuschlag für computer- } \\
\text { gesteuerte Analyse, § } 6\end{array}$ & 0800 & 46,63 \\
\hline 0780A & $\begin{array}{l}\text { Analog: Bougierung der Scheide } \\
\text { entspricht apparative Dehnung } \\
\text { (Sprengung) eines Kardiaspasmus, § } 6\end{array}$ & 0242 & 14,11 \\
\hline $5377 A$ & $\begin{array}{l}\text { Analog: Zuschlag für computergesteu- } \\
\text { erte Analyse und Abbildung an einer } \\
\text { Geburtssimulation an kindlichen und } \\
\text { mütterlichen Maßen entspricht Zuschlag } \\
\text { für computergesteuerte Analyse, §6 }\end{array}$ & 0800 & 46,63 \\
\hline $5377 A$ & $\begin{array}{l}\text { Analog: Zuschlag für 3-D-Rekonstruktion } \\
\text { zusätzlich des Ultraschalls entspricht } \\
\text { Zuschlag für computergesteuerte } \\
\text { Analyse - einschließlich speziell nach- } \\
\text { folgender 3D-Rekonstruktion, §6 }\end{array}$ & 0800 & 46,63 \\
\hline $3652 A$ & $\begin{array}{l}\text { Analog: Messung des Scheiden-pH } \\
\text { entspricht Streifentest im Urin, §6 }\end{array}$ & 0035 & 2,04 \\
\hline $0422 A$ & $\begin{array}{l}\text { Analog: Fetale Zustandsdiagnostik, wie } \\
\text { z. B. fetale Kindsbewegungs- und Atem- } \\
\text { wegsbewegungsanalyse zusätzlich zur } \\
\text { Leistung nach Ziffer } 415 \text { GOÄ entspricht } \\
\text { Eindimensionale echokardiographische } \\
\text { Untersuchung mittels Time-Motion- } \\
\text { Diagramm, mit Bilddokumentation - } \\
\text { ggf. einschließlich gleichzeitiger } \\
\text { EKG-Kontrolle, § } 6\end{array}$ & 0200 & 11,66 \\
\hline $0021 A$ & $\begin{array}{l}\text { Pränataldiagnostische Beratung ent- } \\
\text { spricht humangenetische Beratung, } § 6\end{array}$ & 0360 & 20,98 \\
\hline
\end{tabular}

Untersuchung 30. SSW: Sonographie/Pränataldiagnostik

- 3. Ultraschallscreening, Doppler

- ggf. Echokardiographie

Untersuchung 32.SSW: Sonographie

- Fetometrie, Doppler, FW-Menge

Untersuchung 34. SSW: Sonographie

- Fetometrie, Doppler, FW-Menge

Untersuchung 36. SSW: Sonographie

- Fetometrie, Doppler, FW-Menge

Untersuchung 38. SSW: Sonographie

- Fetometrie, Doppler, FW-Menge

Untersuchung 40. SSW: Sonographie

- Fetometrie, Doppler, FW-Menge

Überwachung über Termin: Sonographie

(Bis maximal 14 Tage bei unauffälliger Schwangerschaft bis zum

7. Tag über Termin zweitägig, dann täglich)

- Bei Erstvorstellung: Fetometrie, Doppler, FW-Menge, dann bei jeder weiteren Vorstellung: FW-Menge und Doppler A. umbilicalis

\section{Stationäre Patientinnen}

Stationäre Patientinnen erhalten mindestens alle 10 Tage eine Fetometrie und eine Doppleruntersuchung. Bei fetaler Risikosituation großzügige Indikation zur Vorstellung in der Pränataldiagnostik.

\section{Zeitlicher Ablauf der Schwangerschaftsvorsorge und Ultraschalluntersuchung \\ Erstuntersuchung}

Anamnese:

- Familienanamnese

- Eigenanamnese

- Schwangerschaftsanamnese (LP, Gestationsalter, frühere Schwangerschaften)

- Arbeits- und Sozialanamnese

Allgemeinuntersuchung:

- Perkussion und Auskultation von Herz und Lunge

- Inspektion und Palpation der Brust

- Palpation des Abdomens und Beurteilung der Druckpunkte

- Inspektion der Haut

Vaginale Untersuchung:

- Cervixscore

- PAP-Abstrich

- vaginaler pH (ggf. mikrobiologischer Abstrich)

Allgemeines Screening:

- Gewichtskontrolle

- Blutdruckmessung
Untersuchung 24. SSW: Sonographie

- Fetometrie und maternaler Doppler FW-Menge
- Fetometrie, ggf. Amniocentese

- 2. Ultraschallscreening

(Fehlbildungsultraschall + Echokardiographie) 
- Untersuchung des Mittelstrahlurins (Eiweiß, Zucker, Sediment, Erythrozyten)

- Hb-Bestimmung (i.d.R. regelmäßig ab dem 6. Monat, falls Erstbestimmung normal)

- ggf. Schwangerschaftstest, HCG

Beratung:

- Ablauf der Schwangerschaftskontrollen

- Berufstätigkeit

- Sport

- Reisen

- Geschlechtsverkehr

- Haustiere

- Ernährung, Substitutionstherapie (Folsäure, Jodid)

- Rauchen, Alkohol, Drogen

Spezielle Beratung:

- Überweisung zur humangenetischen Beratung

- Pränataldiagnostik(s.u.)

Serumscreening:

- Blutgruppe und Rh-Faktor

- Antikörpersuchtest

- Infektionsscreening (Chlamydien, Lues, Röteln, HIV, Toxoplamose

Sonographie:

- Sicherung einer intrauterinen Schwangerschaft

- Bestimmung des Gestationsalters und Überprüfung des mutmaßlichen Entbindungstermins

Mutterpass:

- Ausstellen des Mutterpasses

Untersuchung 12. SSW

Allgemeines Screening:

- Gewichtskontrolle

- Blutdruckmessung

- Untersuchung des Mittelstrahlurins (Eiweiß, Zucker, Sediment, Erythrozyten)

Beratung:

- Schwangerschaftsbeschwerden (Nausea und Erbrechen, Ptyalismus gravidarum, Sodbrennen, Haar- und Hautveränderungen, Wadenkrämpfe, etc.

Varikose, Hämorrhoiden, Obstipation)

Vaginale Untersuchung:

- Cervixscore

- vaginaler $\mathrm{pH}$ (ggf. mikrobiologischer Abstrich)

Sonographie:

- Bestimmung des Gestationsalters und Überprüfung des mutmaßlichen Entbindungstermins

- Mehrlinge (Chorionizität)

- Pränataldiagnostik: 1. Trimester-Screening (NT, $\beta$-hCG \& PAPP-A)

- bei Auffälligkeiten invasive Diagnostik anbieten (AC oder CVS)
Diabetes-Screening:

- Bei entsprechender Risikokonstellation OGTT (75 g)

\section{Untersuchung 16.SSW}

Allgemeines Screening:

- Gewichtskontrolle

- Blutdruckmessung

- Untersuchung des Mittelstrahlurins (Eiweiß, Zucker, Sediment, Erythrozyten)

- Fundusstand

- Herzaktionen (Sono), Kindsbewegungen

Vaginale Untersuchung:

- Cervixscore

- vaginaler pH (ggf. mikrobiologischer Abstrich)

Serumscreening:

- Bei unklarem oder nicht vorhandenem Rötelnschutz Rötelntiter

Beratung:

- Spezielle Fragen

- Angebot des späten Triple-Tests, falls noch nicht erfolgt

Pränataldiagnostik:

- ggf. Amniocentese

- ggf. frühe Fehlbildungsdiagnostik (bei auffälligem Sono in der 12. SSW)

\section{Untersuchung 20. SSW}

Allgemeines Screening:

- Gewichtskontrolle

- Blutdruckmessung

- Untersuchung des Mittelstrahlurins (Eiweiß, Zucker, Sediment, Erythrozyten)

- Fundusstand

- Herzaktionen (Sono), Kindsbewegungen

Vaginale Untersuchung:

- Cervixscore

- vaginaler pH (ggf. mikrobiologischer Abstrich)

Serumscreening:

- ggf. Wiederholung Toxoplasmosescreening

Beratung:

- Spezielle Fragen

Sonographie:

- 2. Ultraschallscreening

Pränataldiagnositk:

- Fehlbildungsultraschall einschließlich maternalem Doppler + fetaler Echokardiographie

- ggf. Wunsch 3D-Ultraschall 
Untersuchung 24. SSW

Allgemeines Screening:

- Gewichtskontrolle

- Blutdruckmessung

- Untersuchung des Mittelstrahlurins (Eiweiß, Zucker, Sediment, Erythrozyten)

- Fundusstand

- Herzaktionen (Sono), Kindsbewegungen

- Hb-Kontrolle

Vaginale Untersuchung:

- Cervixscore

- vaginaler pH (ggf. mikrobiologischer Abstrich)

Beratung:

- Spezielle Fragen

Diabetes-Screening:

- oGTT (75 g)

Sonographie:

- ggf. maternaler Doppler (falls auffällig in der 20. SSW)

Serumscreening:

- Antikörpersuchtest

Untersuchung 28. SSW

Allgemeines Screening:

- Gewichtskontrolle

- Blutdruckmessung

- Untersuchung des Mittelstrahlurins

(Eiweiß, Zucker, Sediment, Erythrozyten)

- Fundusstand

- Kindslage

- Hb-Kontrolle

Vaginale Untersuchung:

- Cervixscore

- vaginaler pH (ggf. mikrobiologischer Abstrich)

Beratung:

- Spezielle Fragen, Empfehlung zur SchwangerschaftsGymnastik

CTG

Prophylaxe:

- 300 ng Anti-D-Immunglobulin bei rh-negativen Müttern, falls im Antikörpersuchtest keine Anti-D-AK nachweisbar waren

Sonographie:

- ggf. feto-maternaler Doppler

\section{Untersuchung 30. SSW}

Allgemeines Screening:

- Gewichtskontrolle

- Blutdruckmessung

- Untersuchung des Mittelstrahlurins (Eiweiß, Zucker, Sediment, Erythrozyten)

- Fundusstand
- Kindslage

- Hb-Kontrolle

Vaginale Untersuchung:

- Cervixscore

- vaginaler pH (ggf. mikrobiologischer Abstrich)

Beratung:

- Spezielle Fragen

CTG

Sonographie:

- 3. Ultraschallscreening

- feto-maternaler Doppler

Pränataldiagnostik:

- ggf. fetale Echokardiotokographie

\section{Untersuchung 32. SSW}

Allgemeines Screening:

- Gewichtskontrolle

- Blutdruckmessung

- Untersuchung des Mittelstrahlurins (Eiweiß, Zucker, Sediment, Erythrozyten)

- Fundusstand

- Kindslage

- Hb-Kontrolle

Serumscreening:

- ggf. Wiederholung Toxoplasmosescreening

Vaginale Untersuchung:

- Cervixscore

- vaginaler pH (ggf. mikrobiologischer Abstrich)

Beratung:

- Spezielle Fragen

CTG

Diabetes-Screening:

- Bei entsprechender Risikokonstellation oGTT (75 g), z. B. 24. SSW oGTT o. B., aber makrosomer Fet

Sonographie:

- ggf. feto-maternaler Doppler

Untersuchung 34. SSW

Allgemeines Screening:

- Gewichtskontrolle

- Blutdruckmessung

- Untersuchung des Mittelstrahlurins (Eiweiß, Zucker, Sediment, Erythrozyten)

- Fundusstand

- Kindslage

- ggf. Hb-Kontrolle

Serumscreening:

- HbsAg bestimmen 
Vaginale Untersuchung:

- Cervixscore

- vaginaler pH (ggf. mikrobiologischer Abstrich)

Beratung:

- Spezielle Fragen

CTG

Sonographie:

- Fetometrie

- ggf. feto-maternaler Doppler

Untersuchung 36. SSW

Allgemeines Screening:

- Gewichtskontrolle

- Blutdruckmessung

- Untersuchung des Mittelstrahlurins

(Eiweiß, Zucker, Sediment, Erythrozyten)

- Fundusstand

- Kindslage

- ggf. Hb-Kontrolle

Vaginale Untersuchung:

- Cervixscore

- vaginaler pH (ggf. mikrobiologischer Abstrich)

Beratung:

- Spezielle Fragen (Entbindungsmodus)

CTG

Sonographie:

- Fetometrie

- ggf. fetaler Doppler

Untersuchung 38. SSW

Allgemeines Screening:

- Gewichtskontrolle

- Blutdruckmessung

- Untersuchung des Mittelstrahlurins (Eiweiß, Zucker, Sediment, Erythrozyten)

- Fundusstand

- Kindslage

- ggf. Hb-Kontrolle

Vaginale Untersuchung:

- Cervixscore (führendes Kindsteil fest?)

Beratung:

- Spezielle Fragen (Entbindungsmodus)
Untersuchung 40. SSW

Allgemeines Screening:

- Gewichtskontrolle

- Blutdruckmessung

- Untersuchung des Mittelstrahlurins (Eiweiß, Zucker, Sediment, Erythrozyten)

- Fundusstand

- Kindslage

- ggf. Hb-Kontrolle

Vaginale Untersuchung:

- Cervixscore (führendes Kindsteil fest?)

Beratung:

- Spezielle Fragen (Entbindungsmodus, Übertragung)

CTG

Sonographie:

- Fetometrie

- Dopplersonographie (A. umbilicalis)

\section{Überwachung über Termin}

(Bis maximal 14 Tage bei unauffälliger Schwangerschaft bis zum 7. Tag über Termin zweitägig, dann täglich)

Allgemeines Screening:

- Gewichtskontrolle

- Blutdruckmessung

- Untersuchung des Mittelstrahlurins (Eiweiß, Zucker, Sediment, Erythrozyten)

- Fundusstand

- Kindslage

Vaginale Untersuchung:

- ggf. Cervixscore (führendes Kindsteil fest?)

Beratung:

- Spezielle Fragen (Entbindungsmodus, Übertragung)

CTG

Sonographie:

- FW-Menge

- Dopplersonographie (A. umbilicalis)

Die Frequenz der Ultraschalluntersuchungen hängt im Einzelfall von der medizinischen Indikationsstellung und der Notwendigkeit ab.

CTG

Sonographie:

- Fetometrie

- ggf. fetaler Doppler 